\title{
The interstellar medium surrounding the Scorpius-Centaurus association revisited ${ }^{\star}$
}

\author{
W. G. L. Pöppel ${ }^{1}$, E. Bajaja ${ }^{1}$, E. M. Arnal ${ }^{1,2, \star \star}$, and R. Morras ${ }^{1,2, \star \star}$ \\ 1 Instituto Argentino de Radioastronomía (CCT La Plata - CONICET), C.C. No. 5, 1894 Villa Elisa, B.A., Argentina \\ e-mail: wpoppel@iar.unlp.edu.ar \\ 2 Facultad de Ciencias Astronómicas y Geofísicas Universidad Nacional de La Plata, Paseo del Bosque s/n, 1900 La Plata, Argentina
}

Received 4 November 2008 / Accepted 4 September 2009

\begin{abstract}
Aims. We want to make a large-scale study of the morphology, kinematics, and origin of the HI, which surrounds the Sco-Cen association.

Methods. We combine our high-sensitivity southern H I survey with the Leiden/Dwingeloo Survey, considering $l=240^{\circ}-400^{\circ}$, $b=-60^{\circ}$ to $+60^{\circ}$, and radial velocities of $V=-41.8$ to $+40.8 \mathrm{~km} \mathrm{~s}^{-1}$. We point out the main $\mathrm{H}$ I branches surrounding the association and derive their kinematics. Kinematical H I-maps were compared with spatial maps of interstellar (IS) Na I from the literature. Upper limits for distances $d$ were derived from optical IS absorption components from the literature. Models of expanding spherical H I shells were fitted around each stellar subgroup.

Results. The expanding ring of $\mathrm{HI}$ associated with the Gould Belt (GB) is very prominent. At each $l$, its radial velocity shifts $\sim-7 \mathrm{~km} \mathrm{~s}^{-1}$ within an interval $\Delta b \sim 10^{\circ}-25^{\circ}$. On the sky, the shifts occur within a narrow stripe extending between $l, b \sim 250^{\circ},-18^{\circ}$ and $400^{\circ},+50^{\circ}$. The ranges of distances and radial thicknesses of most $\mathrm{H}$ I branches are nearly $70-160 \mathrm{pc}$ and $40-90 \mathrm{pc}$, respectively. The shell-models fit the main branches. Interactions between the shells built a large expanding bubble with a transverse diameter of nearly $250 \mathrm{pc}$ around the association. The near face is approaching with a mean velocity $\bar{V} \sim-6.6 \mathrm{~km} \mathrm{~s}^{-1}$ at $d \leq 76 \mathrm{pc}$ from the Sun, covering about $102^{\circ} \times 65^{\circ}(l, b)$, forming an "H I-wall". There are streamers at $\bar{V} \sim-15$ to $-35 \mathrm{~km} \mathrm{~s}^{-1}$, as well as gas presumably overshot into Galactic Quadrant II. The receding gas is more tenuous.

Conclusions. The association is traversing the ring since a time comparable to its age producing a significant disturbance on the expanding GB-ring of gas. The latter was almost totally shocked by the association, northerly of the stripe of velocity shifts. Southerly there are large amounts of preshocked gas, as well as smaller more localized shocked regions. Hot gas within the bubble produces most of the $1 / 4 \mathrm{keV}$ radiation detected toward it by ROSAT. The total mass of the GB gas embedding the Sco-Cen association is estimated at $M_{\mathrm{t}} \sim 368000 M_{\odot} \pm 60 \%$, including $\sim 34000 M_{\odot}$ of associated $\mathrm{H}_{2}$ and $30 \%$ of $\mathrm{He}$. About $39 \%$ of the embedding gas was shocked by the association. At $|b| \geq 35^{\circ}$, a comparison of the observations with test points moving on ballistic orbits is consistent with the formation of the Sco-Cen association within the gas ring of the GB and the presumable explosive origin of the latter. The rotation of the ring is assumed.
\end{abstract}

Key words. open clusters and associations: individual: Sco-Cen association - open clusters and associations: individual: Gould Belt - ISM: clouds -

\section{Introduction}

The Scorpius-Centaurus association is the nearest OB association. It spans about $75^{\circ}$ in $l$, and $40^{\circ}$ in $b$. Blaauw (1964) described it as formed by three subgroups gravitationally unbounded: Upper Scorpius (US), Upper Centaurus Lupus (UCL), and Lower Centaurus Crux (LCC). The star formation was sequential, starting at UCL and/or LCC, and propagating later to US (Blaauw 1991; de Geus 1992). The well-known star $\zeta$ Oph should be a run-away, probably from US (Hoogerwerf et al. 2000). Large populations of pre-main-sequence stars (PMSs) have been detected in the three subgroups (e.g. Preibisch et al. 1998, 2002; Mamajek et al. 2002; Slesnick et al. 2008).

The Sco-Cen association is thought to belong to the Gould Belt (GB, e.g. Blaauw 1991; de Zeeuw et al. 1999). This is a flat local system of stars younger than about $7 \times 10^{7} \mathrm{yr}$, tilted

* Appendix is only available in electronic form at http://www . aanda.org

$\star \star$ Member of the Carrera del Investigador Científico, CONICET, Argentina. at nearly $18^{\circ}$ to the Galactic plane, and at distances not greater than about $600 \mathrm{pc}$. The GB is associated with large amounts of cold H I ("feature A", see Lindblad 1967) and several molecular cloud complexes (Dame \& Thaddeus 1985). On $V-l$ and $V-$ $b$ contour maps at the $21-\mathrm{cm}$ line, the local HI appears as a narrow ridgelike structure, whose main component is the cold gas associated with the GB (e.g. Pöppel 1997). At low $|b|$, this cold HI was modeled as an expanding ring, which surrounds the Sun eccentrically (Lindblad et al. 1973; Olano 1982; Perrot \& Grenier 2003, in the following P\&G). The likely distribution of the H I was mapped by Pöppel et al. (1994) at $|b| \geq 10^{\circ}$.

Weaver (1979) concluded that a large bubble of interstellar (IS) matter is enclosing the Sco-Cen association, and de Geus (1992) made a very schematic plot of three loops or shells of $H I$, each one associated with a stellar subgroup. He estimated the total H I mass at $4.8 \times 10^{5} M_{\odot}$, the kinetic energy of the shells at $7.7 \times 10^{50} \mathrm{erg}$, and the total energy output of the association since its origin at nearly $16 \times 10^{50} \mathrm{erg}$, which appeared as sufficient to form the shells. Egger \& Aschenbach (1995) pointed out a prominent X-ray shadow from a dense, ringlike 
H I feature. They interpreted it as a wall of HI, which resulted from a collision between a Loop I superbubble around the ScoCen association and the hot Local Bubble. The wall of H I would be located between both bubbles at about $100 \mathrm{pc}$ from the Sun. Egger (1998) suggested a "local interstellar foam", which could be associated with the GB. However, Egger \& Aschenbach's scenario makes no consideration of the kinematics of the HI. Furthermore, their ringlike feature has been questioned (Reis \& Corradi 2008). On the other hand, from a detailed study involving about 3900 OB stars Fernández (2005; et al. 2008) concluded that the Sco-Cen association could have formed in the Sgr-Car arm and not in the GB. Very recently, Ortega et al. (2009) have investigated the role played by the LCC, and UCL subgroups in the formation of several young nearby stellar associations mainly composed of PMSs.

Thus, it is not obvious that the H I surrounding the Sco-Cen association originated in the expanding ring associated with the GB. Actually, neither de Geus (1992) nor Ortega et al. (2009) evaluated this possibility, whereas Heiles (1998) expressed his doubts, even about the existence of the expanding ring. In contrast, Pöppel \& Marronetti (2000, from here on P\&M) conclude that a significant disturbance of the H I-ring is expected in this region due to the star formation processes in the association. Therefore, our aims are the following: i) to make a new detailed large-scale study of the spatial distribution and kinematics of the main H I branches and features surrounding the Sco-Cen association, ii) to check any relation of them with the Sco-Cen association, and with the GB, if any, iii) to check the presence of the shells in more detail, and iv) to check an adequate scenario. We use a new very homogeneous and sensitive $21-\mathrm{cm}$ line data base, combined with the results of optical observations taken from the literature.

The paper is organized as follows. In Sect. 2 we make a short description of the new $21-\mathrm{cm}$ line data base. In Sect. 3.1 we point out the main H I branches, as well as the smaller features, which are around the Sco-Cen association, by means of sky maps at different ranges of the radial velocity $V$. We also compare them with an IRAS map at $100 \mu \mathrm{m}$. In Sect. 3.2 we focus on the kinematics of the H I by considering $V-b$ contour maps at fixed $l$. In Sect. 4.1 we derive the distance ranges of some of the $\mathrm{H}$ I features by comparisons with maps of the spatial distribution of the IS Na I derived by Vergely et al. (2001) and Lallement et al. (2003). In Sect. 4.2 we derive upper limits for the distances by means of IS lines from optical data taken from the literature. In Sect. 5 we focus on the HI shells described schematically by de Geus. We make new fits and analyze their kinematical characteristics. In Sects. 6.1-4 we discuss the results considering the distances, the formation of a bubble, its physical characteristics, and a scenario. In Sect. 7 we summarize our conclusions.

\section{H I observations: High-sensitivity all-sky survey}

In this paper we are using our new high-sensitivity southern 21-cm line survey (see Arnal et al. 2000), combined with the Leiden/Dwingeloo Survey of Galactic Neutral Hydrogen (Hartmann \& Burton 1997). The resulting grid has a spacing $(\Delta l, \Delta b)=\left(0.50^{\circ}, 0.50^{\circ}\right)$. The velocity range is $-450 \mathrm{~km} \mathrm{~s}^{-1}$ to $+400 \mathrm{~km} \mathrm{~s}^{-1}$, spaced at $1.03 \mathrm{~km} \mathrm{~s}^{-1}$. The angular and velocity resolutions are about $30^{\prime}$ and $1.3 \mathrm{~km} \mathrm{~s}^{-1}$, respectively. The mean rms noise is $0.07 \mathrm{~K}$. The accuracy of the brightness temperature scale is estimated at $5 \%$. Neither discontinuities nor incoherencies appear to exist between the northern and the southern data sets (see Bajaja et al. 2000; Kalberla et al. 2004). No correction for stray radiation was applied to the southern HI data used in this paper since we deal only with the relatively intense features of large angular scales.

We derived HI maps, which show the distribution of the brightness temperature $T_{\mathrm{b}}$ as a function of $l, b$, and the radial velocity $V$, which refers to channel centers. We focus on the region $l=240^{\circ}-400^{\circ}, b=-60^{\circ}$ to $+60^{\circ}$ at $|V|<40 \mathrm{~km} \mathrm{~s}^{-1}$. For computational reasons, we write the values of $l$ between $0^{\circ}$ and $40^{\circ}$ as between $360^{\circ}$ and $400^{\circ}$. Unless stated otherwise, the velocities refer to the LSR, which is defined by a solar velocity $V_{\odot}=20 \mathrm{~km} \mathrm{~s}^{-1}$ toward $l_{\mathrm{o}}=56.2^{\circ}, b_{\mathrm{o}}=+22.8^{\circ}$.

\section{The $\mathrm{HI}$ surrounding the Sco-Cen association}

\subsection{I- b maps: conspicuous elongated HI features}

As a first insight into the HI surrounding the Sco-Cen association in Figs. 1-4 we show four rectangular $l-b$ maps covering the range of radial velocities $V$ between -41.2 and $+40.2 \mathrm{~km} \mathrm{~s}^{-1}$ in adjacent intervals. The most intense $\mathrm{HI}$ features appear at the low velocities in Figs. 2 and 3, whereas at the intermediate velocities (IVs) in Figs. 1 and 4, the features are less intense. We quote that the positive velocities (Figs. 3, 4) denote peculiar motions for nearby objects in the Galactic quadrant (GQ) IV. In Fig. 3 the US subgroup is enclosed by the wellknown US-loop, whereas the UCL and LCC subgroups lie in a region of low column densities $N_{\mathrm{HI}}$, which is adjacent to a large hole contained approximately within the area $l, b \sim 280^{\circ}-330^{\circ}$, $+15^{\circ}$ to $+40^{\circ}$. Notice the large dense triangular clump toward the molecular Aql Rift in the GQI (see Table 4 in Sect. 6.2).

To perform a study and to derive physical parameters, in each figure we selected those features, which appeared the most conspicuous, identifying them by capital letters and drawing heavy lines schematically. We are conscious that our selection and drawings can be considered as non unique, especially regarding the less intense features, some of which should be considered merely as samples of H I enhancements. In spite of this, notice that the large arclike branches or filaments $A_{1}-A_{2}, B_{1}-B_{2}$, $\mathrm{C}$, and LCC in Fig. 2, and the US-loop in Fig. 3 are qualitatively similar to the positions of the shells drawn schematically by de Geus (1992) in his Fig. 1b without kinematical references. Only the southern filamentary features $\mathrm{D}_{0}, \mathrm{D}_{1}$, and $\mathrm{D}_{2}$ show significant differences with de Geus' most southern shell. We come back to the meaning of these features in Sect. 6.2. Moreover, in Fig. 4 the IV branches $\mathrm{M}_{1}-\mathrm{M}_{2}$ correspond to $\mathrm{HI}$, which was studied by Fejes (1971).

Figure 5 shows the superposition of a low-resolution IRAS map at $100 \mu \mathrm{m}$ and an H I map integrated over $V=-19.6$ to $+19.6 \mathrm{~km} \mathrm{~s}^{-1}$. After excluding the dense regions near to the Galactic plane at $|b| \leq 10^{\circ}$, both maps show a close global similarity. Most of the intense features pointed out in Figs. 1-4 are apparent in the IRAS map, including the large hole. Toward the US-subgroup the relative intensity of the emission is much higher in the $100-\mu \mathrm{m}$ map than in the $21-\mathrm{cm}$ map. This suggests a higher temperature of the dust in this region, because of the bright radiation field of the young stars and the presence of several well-known HII regions (see Sect. 5, ii). The close global correlation between the H I and the $100-\mu \mathrm{m}$ maps is consistent with the absence of very large molecular cloud complexes related to the Sco-Cen association (see Table 4). It also implies that gas and dust are well mixed.

Table 1 summarizes the main parameters of each subgroup of the association. They will be of interest for us in the next sections. The meaning of the columns is the following: $1-4)$ the name, the range in $l, b$, and the mean distance 
W. G. L. Pöppel et al.: The interstellar medium surrounding the Scorpius-Centaurus association revisited

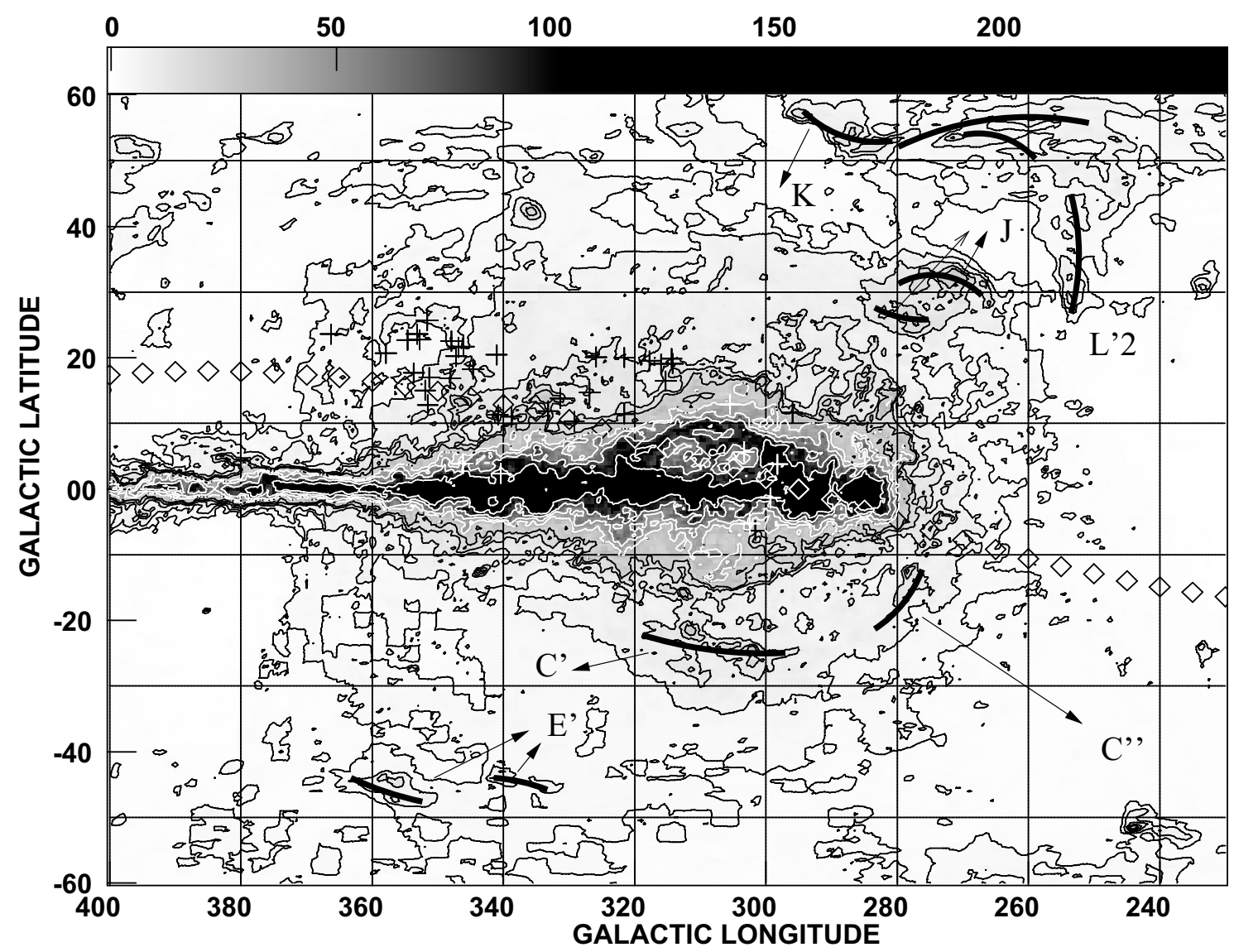

Fig. 1. Gray-scale map for H I with the channels integrated over $V=-41.2$ to $-20.6 \mathrm{~km} \mathrm{~s}^{-1}$ and a scale flux ranging from 0 to $100 \mathrm{~K}$. The levels are $2.48 \times(1,2,4,6,8,14,20,30$, and 45). The selected branches are indicated schematically. The crosses indicate the stars of spectral types OB2.5 of the Sco-Cen association (de Bruijne 1999), including $\zeta$ Oph. The diamonds indicate the plane of the GB (Stothers \& Frogel 1974). See the text for more details.

(de Zeeuw et al. 1999); 5) the characteristic depth along the line of sight (de Bruijne 1999); 6), 7) and 8) the averages of the radial velocity and of the components of the tangential velocity in the directions of $l$ and $b$, respectively. They were reduced to the LSR using the mean values of the coordinates, distances, and proper motions (de Zeeuw et al. 1999); 9) the nuclear age (de Geus 1992); 10), and 11), the numbers of stars of spectral type B and earlier than B2.5, respectively (de Bruijne 1999); $12), 13)$, and 14) the numbers of exploded supernovae (SN) according to Blaauw (1991); de Geus (1992); and Maíz-Apellániz (2001), respectively; 15) the total stellar masses (de Geus 1992).

\section{2. $V-b$ contour maps: The $\mathrm{HI}$ ridge at low velocities and the kinematics of the HI features}

To obtain a global insight into the kinematics of the H I features, we sampled the data by means of $65 l$-slices in the region $l=240^{\circ}-400^{\circ}$, spaced at intervals of $2.5^{\circ}$. In all these kinematical $V-b$ contour maps, the most prominent feature is the intense clumpy $H$ I ridge centered at low velocities $V_{R}$, whose main component is the cold gas associated with the GB (see Sect. 1).

All the features pointed out in Figs. 1-4 can be tracked more precisely in the $65 \mathrm{~V}-b$ maps. For a given feature, its radial peak velocity $V_{\mathrm{p}}$ was derived in each $V-b$ map, where the feature could be detected. The results were plotted in Fig. 6. The plots were grouped according to the kinematical characteristics of the features namely, some features of $V_{\mathrm{p}}<0$, and other ones of $V_{\mathrm{p}}>0$ in panels I and II, respectively; those of IVs and branch $\mathrm{E}$ in panel III, and some selected features in panel IV. Two or more values of $V_{\mathrm{p}}$ for one $l$ correspond to double or multiple clumps. The mean uncertainties of $V_{\mathrm{p}}$ are estimated at about $1 \mathrm{~km} \mathrm{~s}^{-1}$, but for weak or blended features the uncertainties might be considerably larger. The plots will be considered in the next sections.

The thermal conditions of the main component of the $\mathrm{HI}$ ridge were verified by sampling its full velocity widths at half intensity $\Delta V$. Considering contours of $T_{\mathrm{b}} \geq 12 \mathrm{~K}$, we selected simple peaks at $|b| \geq 15^{\circ}$ for avoiding the blending by the Galactic background. The measured widths $\Delta V_{\mathrm{c}}$ are upper limits for $\Delta V$, since no correction for background radiation was made. Assuming a Gaussian velocity distribution, the upper limits for the radial-velocity dispersions are $\sigma_{V_{\max }} \simeq \Delta V_{\mathrm{c}} / 2.35$.

Two cases were considered according to the positions selected on the ridge, namely, i) on any one of the features pointed out in Figs. 2, 3, and ii) on no particular positions. In both cases the results were similar. No significant differences were obtained between positive and negative $b$. The average for 96 positions was $\bar{\sigma}_{V_{\max }}=4.3 \pm 1.4 \mathrm{~km} \mathrm{~s}^{-1}$. This value is characteristic of the cold neutral medium (CNM). A new check was made at 118 positions at $|b| \geq 15^{\circ}$ using a sample of $V-l$ contours. The results did not change significantly, so the main component of the $\mathrm{HI}$ ridge and of the selected features around the Sco-Cen association in Figs. 2, 3 is actually turbulent cold HI.

Furthermore, a remarkable characteristic of the $\mathrm{HI}$ ridge that is apparent in the $l$-slices is a systematic mean-velocity gradient $\Delta \bar{V}_{R} / \Delta b<0$ between both its $b$-extremes. It mainly comes from a striking velocity-shift $\Delta V_{R}<0$ of a few $\mathrm{km} \mathrm{s}^{-1}$ occurring 


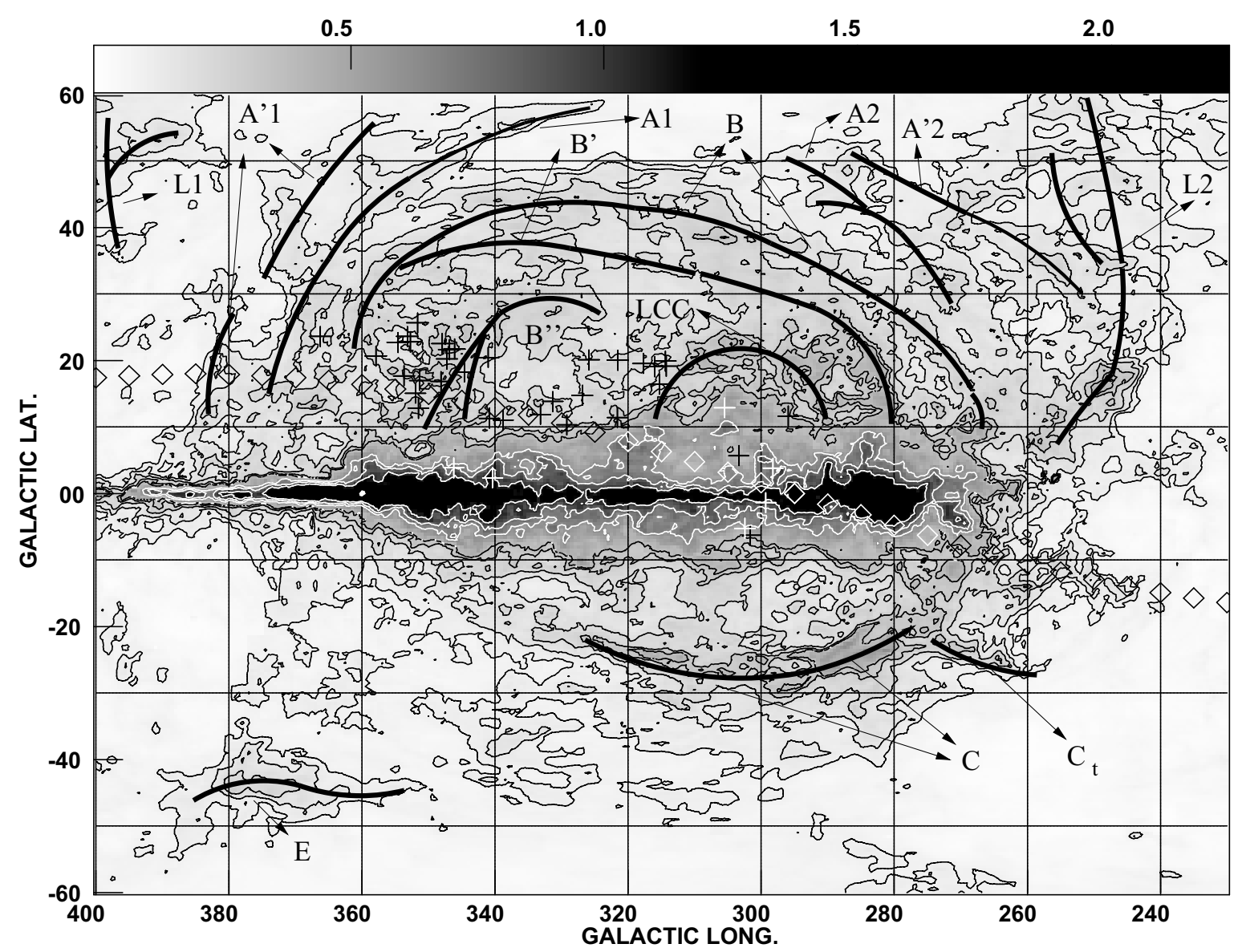

Fig. 2. Gray-scale map for H I with the channels integrated over $V=-19.6$ to $-1.0 \mathrm{~km} \mathrm{~s}^{-1}$ and a scale flux ranging from 0 to $1.2 \mathrm{kilo} \mathrm{K}$. The levels are $22.3 \times(4,6,9,13,16,28,36$, and 48$)$. The selected branches are indicated schematically. For convenience, $\mathrm{B}$ was subdivided into $\mathrm{B}_{1}$, at $l>310^{\circ}$ and $\mathrm{B}_{2}$, at $l<310^{\circ}$. The LCC-arc is curved about a thick LCC-clump. The crosses indicate the stars of spectral types OB2.5 of the Sco-Cen association (de Bruijne 1999), including $\zeta$ Oph. The diamonds indicate the plane of the GB. See the text for more details.

within a latitude interval $\Delta b>0$, having a width of $10^{\circ}-25^{\circ}$. In regard to this, for each $l$ we selected a pair of rather nearby latitudes $b_{1}$ and $b_{2}>b_{1}$, for which the velocity-shift $\Delta V_{R}=V_{R}\left(b_{2}\right)-V_{R}\left(b_{1}\right)<0$ was plain to see. Near to the Galactic plane, the intervals $\Delta b=b_{2}-b_{1}$ were chosen somewhat in excess for avoiding the blending effects by the background. These are most severe at $l<270^{\circ}$, where the main emission from the background is at $V>0$. On the celestial sphere, the locus of the selected widths $\Delta b$ defines a stripe of velocity shifts, which crosses the Sco-Cen association, as shown in Fig. 7.

Figure 8 is a plot of both $V_{R}\left(b_{1}\right)$ and $V_{R}\left(b_{2}\right)$ as functions of $l$. The mean uncertainties are nearly $1 \mathrm{~km} \mathrm{~s}^{-1}$. For the sake of comparison we plotted the mean radial velocities of the stellar subgroups, as well as the velocities of the GB's ring of gas according to P\&G's (2003) fit and to Olano's model 1. As can be seen, within the range $l \sim 270^{\circ}-400^{\circ} \mathrm{P} \& \mathrm{G}$ 's fit is consistent with the southern values $V_{R}\left(b_{1}\right)$, which are positive. Olano's fit is less consistent. At $l<270^{\circ} V_{R}\left(b_{1}\right)$ is in large excess of P\&G's fit. This should be understood in terms of the blending effects by the background gas from the region of the Gum nebula, whose velocities reach up to $+10 \mathrm{~km} \mathrm{~s}^{-1}$ (see Reynoso \& Dubner 1997). In contrast, the northern values $V_{R}\left(b_{2}\right)$, which are predominantly negative, are systematically below the fits,suggesting a disturbance of the expanding ring of the GB gas. The stellar mean radial velocities are systematically above the H I velocities. From 53 maps over the range $l=270^{\circ}-400^{\circ}$, we computed averages $\Delta \bar{V}_{R}=-6.6 \pm 2.4 \mathrm{~km} \mathrm{~s}^{-1}$, and $\overline{\Delta b}=16.1 \pm 7.2^{\circ}$.
A further velocity shift of the ridge is associated with branch $\mathrm{C}$, whose velocities are negative (see Fig. 6, I). In contrast, at nearby latitudes north of branch $\mathrm{C}$, the velocity $V_{R}$ of the ridge is positive and approximately consistent with $\mathrm{P} \& \mathrm{G}$ 's fit within $2 \mathrm{~km} \mathrm{~s}^{-1}$ (see Fig. 6, II). Moreover, at the south of branch $\mathrm{C}$, where the branches $\mathrm{D}_{1,2}$ are located (i.e. at $l=$ $\left.270^{\circ}-350^{\circ}\right), V_{R}$ is also positive but more irregular and only roughly consistent with $\mathrm{P} \& \mathrm{G}$ 's fit (see Fig. 6, IV).

\section{Spatial characteristics of the $\mathrm{HI}$ features}

\subsection{Comparison with the distribution of the IS NaI}

A good tracer of the IS cold $\mathrm{HI}$ is the IS Na I. Figure 9 shows the mean distances $\bar{d}$ of the IS Na I fitted by Lallement et al. to the isoequivalent absorption widths of $50 \mathrm{~m} \AA$ (corresponding to $N_{\mathrm{HI}}>10^{20} \mathrm{~cm}^{-2}$ ) from 426 stars selected near to the Galactic plane (see their Fig. 4). For the sake of comparison, we show the mean distances of the stellar subgroups, the molecular dark cloud complexes (mdccs) related to the GB (see Table 4 in Sect. 6), and GB's ring of gas according to both Perrot \& Grenier's fit and Olano's model 2.

As can be seen, near to the Galactic plane $\bar{d}$ ranges between $\sim 70-225 \mathrm{pc}$. Both the stellar subgroups, and the mdccs have similar mean distances as the IS NaI along $l \sim 300^{\circ}-360^{\circ}$. P\&G's fit for the GB gas is consistent with these mean distances in the range $l \sim 315^{\circ}-360^{\circ}$, with $\bar{d} \sim 150$ pc. In 
W. G. L. Pöppel et al.: The interstellar medium surrounding the Scorpius-Centaurus association revisited

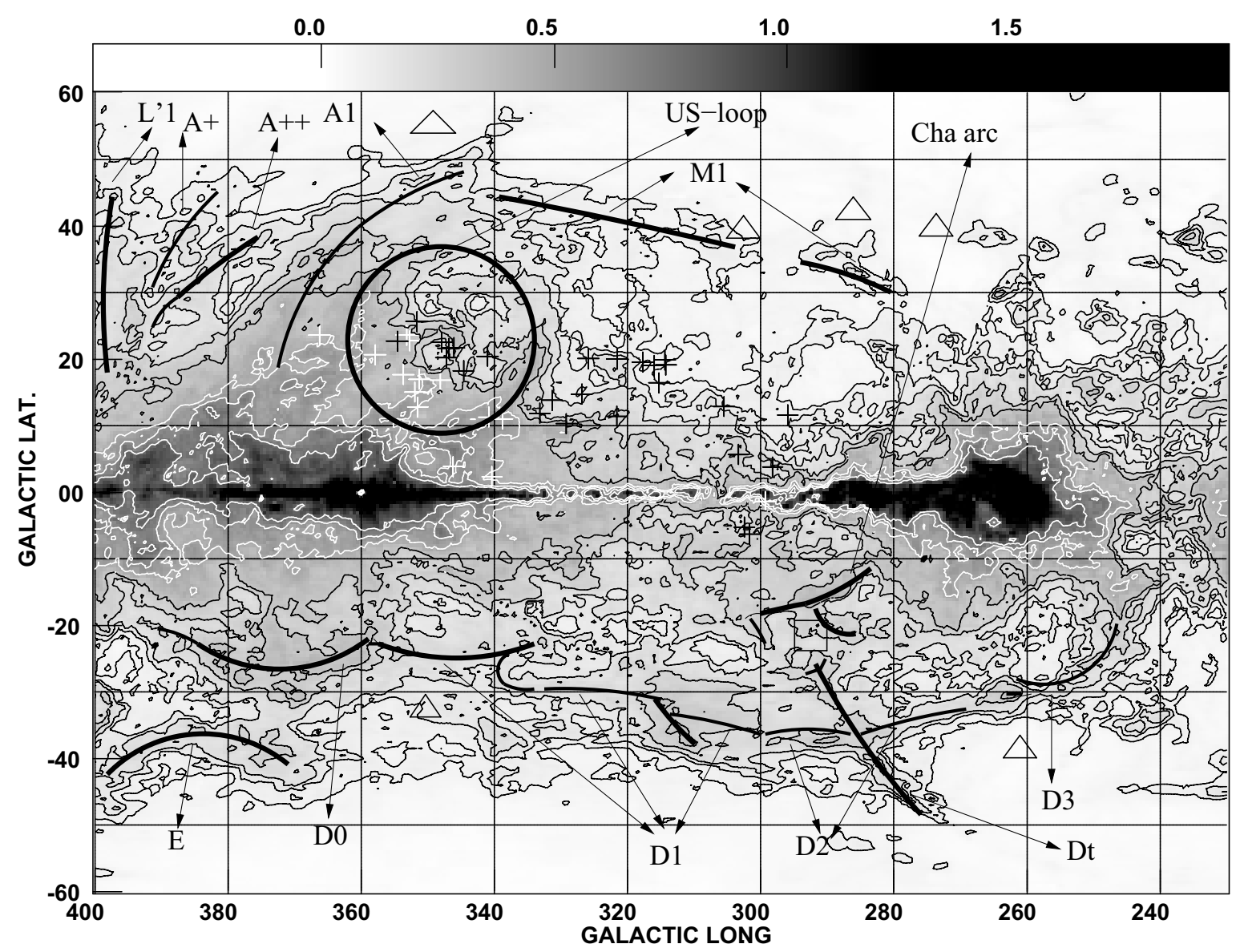

Fig. 3. Gray-scale map for H I with the channels integrated over $V=0.0$ to $+15.5 \mathrm{~km} \mathrm{~s}^{-1}$ and a scale flux ranging from 0 to $1.2 \mathrm{kilo} \mathrm{K}$. The levels are $19.5 \times(4,6,8,12,16,25,32$, and 40$)$. The selected branches are indicated schematically. The extensive wavy branch $\mathrm{D}$ is subdivided into the pieces $\mathrm{D}_{0}, \mathrm{D}_{1}, \mathrm{D}_{2}$, and $\mathrm{D}_{3}$. The crosses indicate the stars of spectral types OB2.5 of the Sco-Cen association (de Bruijne 1999), including $\zeta$ Oph. The open triangles indicate the six sight lines sampled by Sembach et al. (2003), and the open square indicates the small X-ray emitting compact aggregate of PMSs discovered by Mamajek et al. (2000) near to $\eta$ Cha (see Sect. 6.2). See the text for more details.

contrast, $\mathrm{P} \& \mathrm{G}$ 's fit is above $\bar{d}$ systematically by about $10-50 \mathrm{pc}$ at $l \sim 260^{\circ}-315^{\circ}$, and by more than $100 \mathrm{pc}$ at $l>375^{\circ}$. Olano's modeled distances appear to be less consistent with the results.

Figure 7 shows that near to the Galactic plane the GB gas, which has shifted velocities $V_{R}<0$, is present for $l<315^{\circ}$. Negative velocities let us expect a shortening of the original distances of the GB-gas. Actually, the shortenings of the distances suggested by Fig. 9 are consistent with the observed velocityshifts (Figs. 7 and 8 ) and the ages of the stellar subgroups listed in Table 1. Following the suggestion made in Sect. 3.2 we assume that the velocity shifts shown by the H I ridge are disturbances produced on GB's expanding ring of $\mathrm{HI}$ by the most luminous $\mathrm{OB}$ stars of the association. Consequently, in Fig. 8 $V_{R}\left(b_{1}\right)$ corresponds to the largely undisturbed substratum south of the stripe of velocity shifts indicated in Fig. 7, whereas $V_{R}\left(b_{2}\right)$ corresponds to the disturbed one north of the stripe. The large hole at $V>0$ mentioned in Sect. 3 lies north of the stripe.

Our assumption is also consistent with the results of Corradi et al. (2004), who observed IS absorption lines of $\mathrm{NaI}$ in stellar spectra in the region $294^{\circ} \leq l \leq 308^{\circ},-22^{\circ} \leq b \leq+5^{\circ}$. They detected two extended components namely, an intense one along $b \sim-18^{\circ}$ to $+1^{\circ}$, at $d \simeq 120-150$ pc with $V$ decreasing from +3 to $-3 \mathrm{~km} \mathrm{~s}^{-1}$, and a faint one at $d<60 \mathrm{pc}$, with a mean velocity $\bar{V}=-7 \mathrm{~km} \mathrm{~s}^{-1}$. The characteristics of the intense component $\left(\log \mathrm{Na} \mathrm{I} \sim 12.3-13.2 \mathrm{~cm}^{-2}\right)$ are consistent with those shown by the H I ridge. The faint component, whose column densities are below the densities considered in Fig. $9\left(\log \mathrm{NaI} \sim 11.2 \mathrm{~cm}^{-2}\right.$, corresponding to $N_{\mathrm{H}} \sim 3 \times 10^{19} \mathrm{~cm}^{-2}$ ), is consistent with an approaching LCC-shell (see Sect. 5). Thus, from Figs. 7-9, and our assumption we conclude that at present the entire Sco-Cen association is crossing the gas of the expanding ring of the GB.

In order to obtain some information about the spatial distribution of the H I features pointed out in Sect. 3.1, we made a direct comparison of our kinematical maps of H I with the spatial maps of the local IS Na I, as derived by Vergely et al. (2001, their Fig. 5), and Lallement et al. (2003, their Figs. 7 and 8, with more complete data). The maps of $\mathrm{NaI}$ are spaced at $\Delta l=15^{\circ}$ at the same values of $l$ as our $V-b$ maps. Vergely et al. concluded that the modeled features of $\mathrm{Na}$ I describe mainly the gas related to the $G B$. We restricted to $|b| \leq 60^{\circ}$.

In each set of $\mathrm{NaI}$ maps we selected all the modeled dense Na I clumps at each $l$, reading off the ranges of Galactic latitudes $\Delta b_{\mathrm{Na}}$ subtended by their sharp borders. Moreover, in the $V-b$ maps at each $l$ we read off all the identified enhancements embedded in the $\mathrm{H}$ I ridge having $T_{\mathrm{b}} \geq 12 \mathrm{~K}$ as presumable spatial counterparts of the dense Na-I clumps selected at the same $l$ and similar $b$-ranges. Notice that the $\mathrm{HI}$ features refer to $l$-slices having a thickness of $0.5^{\circ}$, whereas the modeled $\mathrm{Na}$ I maps presumably present a larger angular smearing. Furthermore, there are blending effects. Therefore, the assignation of counterparts is 


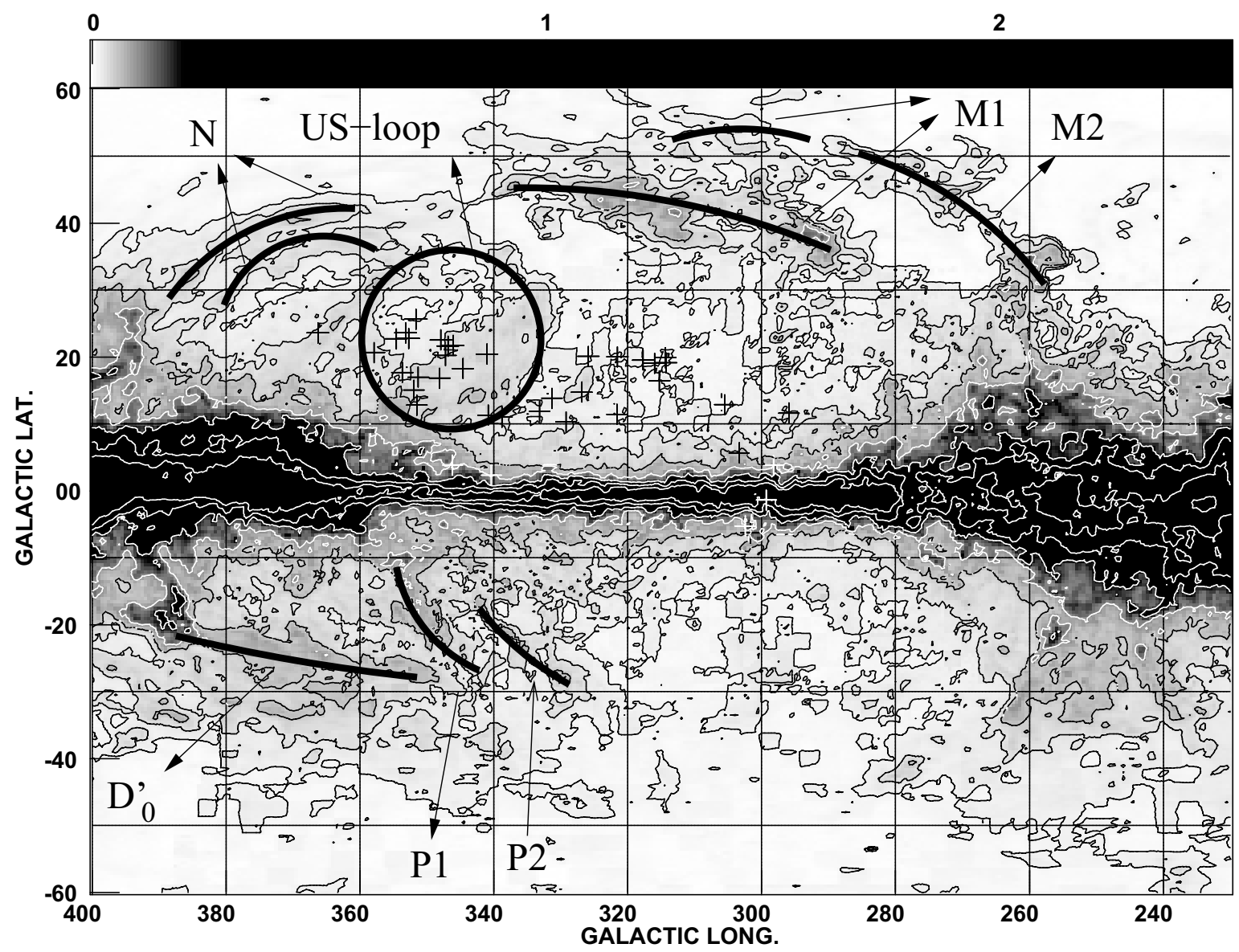

Fig. 4. Gray-scale map for H I with the channels integrated over $V=+16.5$ to $+40.2 \mathrm{~km} \mathrm{~s}^{-1}$ and a scale flux ranging from 0 to $200 \mathrm{~K}$. The levels are $25.1 \times(0.5,1,2,4,8,16$, and 32). The selected branches are indicated schematically. The crosses indicate the stars of spectral types OB2.5 of the Sco-Cen association (de Bruijne 1999), including $\zeta$ Oph. See the text for more details.

not always unambiguous, and some spatial identifications were not possible or might be doubtful. No Na I counterparts could be found for the faint IV features.

The results are shown in Table 2. The HI features pointed out in Figs. 1-4, are listed in two groups, according to the sign predominating in their velocities $V_{\mathrm{p}}$ in Fig. 6. Branch E, which has $V_{\mathrm{p}}$ of both signs, was added at the end. The meaning of the columns is the following: 1) the designation; 2) the mean angular transverse half-width $\bar{\Delta} \theta$, computed from at least three independent estimates on $V-b$ or $V-l$ contours; 3$)$ the mean peak brightness temperature and its dispersion derived from the $V-b$ contours with rough background corrections; 4) and 5), the number $k$ of identifications of an H I feature as a counterpart of a $\mathrm{Na}$ I clump, and the averages of the distance-ranges $\bar{d}_{12}$ covered by the $\mathrm{Na}$ I clumps on the line of sight, as derived from the results of Vergely et al. (2001) and Lallement et al. (2003), respectively. Doubtful values and averages with dispersions greater than $40 \%$ are indicated by a "?". The further columns of Table 2 are considered in the next section.

A comparison of the distance ranges in Cols. 4 and 5 shows differences greater than $50 \mathrm{pc}$ only for branches $\mathrm{B}_{2}, \mathrm{C}, \mathrm{D}_{1}$, and $\mathrm{E}$. The averages of the thicknesses derived from Cols. 4 and 5 are $54 \pm 8 \mathrm{pc}$ and $85 \pm 19 \mathrm{pc}$, respectively, excluding the values marked with a "?", as well as the Aql Rift-H I. The latter has very large thicknesses, suggesting a blending of several H I clouds along the line of sight. The derived averages are of the same order as the expected radial thickness of the GB-ring, which is $\Delta d_{R} \sim \sigma \tau \sim 65 \mathrm{pc}$, where $\sigma \sim 2.5 \mathrm{~km} \mathrm{~s}^{-1}$ is the typical velocity dispersion of feature A (Lindblad 1967), and $\tau \sim 26 \mathrm{Myr}$ its age $(\mathrm{P} \& \mathrm{G})$. The averages are also similar to the characteristic depths $H$ of the stellar subgroups (see Table 1), as well as to their largest initial linear dimensions estimated at nearly $50 \mathrm{pc}$ by Blaauw (1991).

\subsection{Upper limits for the distances from optical IS lines}

We derived upper limits $d_{\max }$ for the distances $d$ of several of the features in Table 2 from a data base of optical IS lines selected from the literature. The lines are mainly from $\mathrm{Na}$ I, K I, Ca II, and Ti II observed in absorption at high resolution in the optical spectra of nearly 330 stars located at distances $d \leq 500 \mathrm{pc}$. When available, we adopted the parallaxes measured by the satellite Hipparcos. The typical errors of the stellar distances are estimated to lie between $\sim 7 \%$ for $d=50-100 \mathrm{pc}$ and $\sim 15 \%$ for $d=200-250 \mathrm{pc}$, with large dispersions. For avoiding lack of sensitivity in the observations we considered only stars having at least one positive detection that is not too weak.

For a given H I feature, we selected all the data available along its lines of sight. Results derived by different authors were included independently. For the detected IS components, we considered the radial velocities $V$ as a function of $d$, with the resolution as an error bar of $V$.

The data used for features of Table 2, as defined by Figs. 1-4, are listed in the Appendix in Table A.1, panels 1-13 (plotted in Fig. A.1) and panels 16, 17 (with no plot). An upper limit was derived from at least two optical detections within the corresponding range of $V_{\mathrm{p}}$ in Fig. 6, adopting the second nearest distance as $d_{\max }$. In some cases an additional IS component was 
W. G. L. Pöppel et al.: The interstellar medium surrounding the Scorpius-Centaurus association revisited

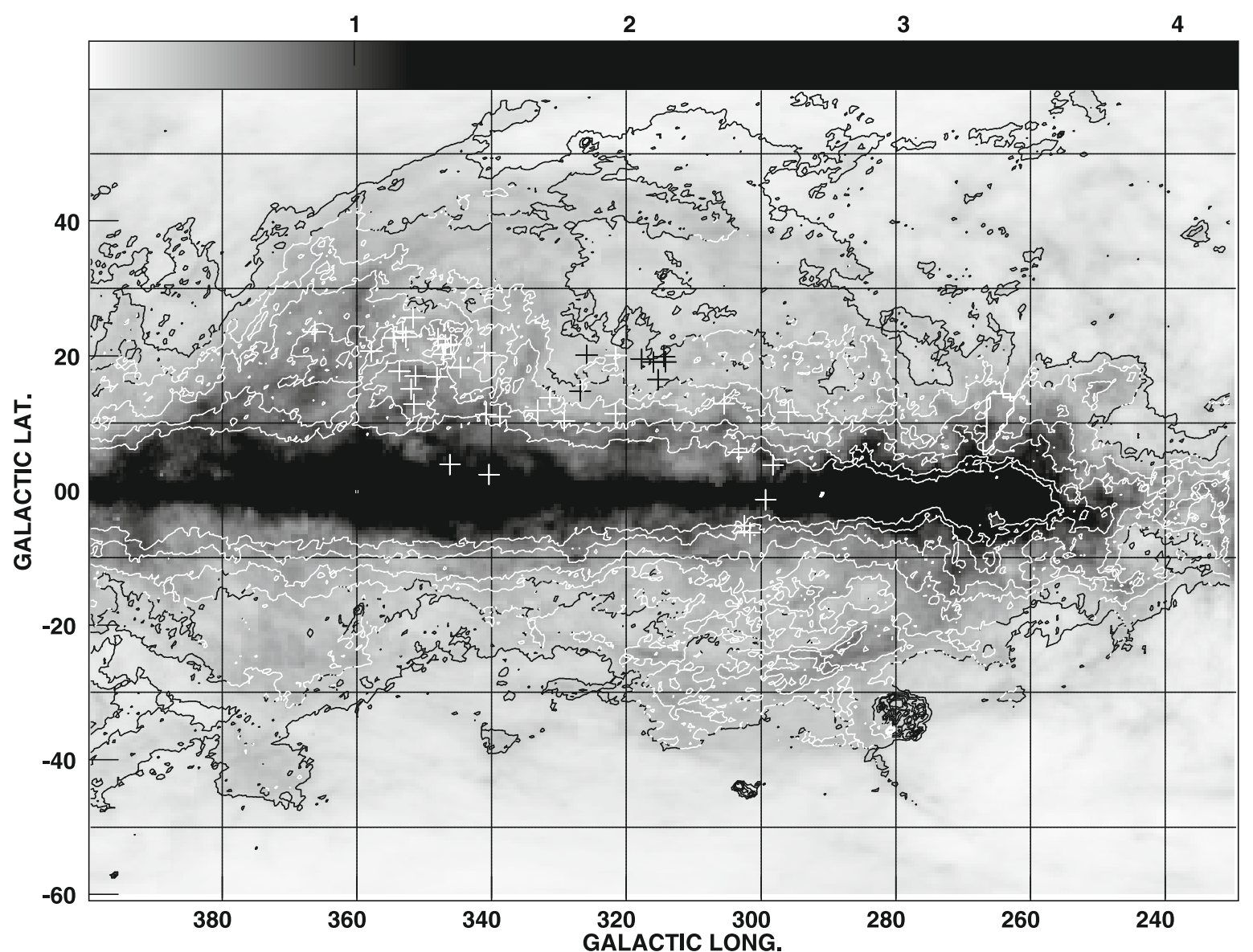

Fig. 5. $100 \mu \mathrm{m}$ contours obtained from the IRAS Sky Survey as produced by the Sky View Survey Analysis System with pixels of $0.22^{\circ} \times 0.22^{\circ}$. The levels are $1.503 \times(3,5,8,12,20$ and 30) MJy/ster. Superposed is a gray-scale map with a flux range 0 to $1200 \mathrm{~K}$, corresponding to the $\mathrm{H}$ I line emission with the channels integrated over $V=-19.6$ to $+19.6 \mathrm{~km} \mathrm{~s}^{-1}$. The crosses indicate the OB2.5 stars of the Sco-Cen association (de Bruijne 1999), including $\zeta$ Oph. The spots at $l, b \sim 279^{\circ},-32^{\circ}$, and $302^{\circ},-45^{\circ}$ correspond to the Magellanic Clouds. See the text for more details.

Table 1. Main parameters of the subgroups of the Sco-Cen association.

\begin{tabular}{|c|c|c|c|c|c|c|c|c|c|c|c|c|c|c|}
\hline $\begin{array}{l}\text { sub- } \\
\text { group } \\
\text { (1) }\end{array}$ & $\begin{array}{l}l \\
\left({ }^{\circ}\right) \\
(2)\end{array}$ & $\begin{array}{l}b \\
\left({ }^{\circ}\right) \\
(3)\end{array}$ & $\begin{array}{l}\bar{d} \\
\mathrm{pc} \\
(4)\end{array}$ & $\begin{array}{c}H \\
\text { pc } \\
(5)\end{array}$ & $\begin{array}{l}\bar{V}_{r} \\
\mathrm{~km} \mathrm{~s}^{-1} \\
(6)\end{array}$ & $\begin{array}{l}\bar{V}_{l} \\
\mathrm{~km} \mathrm{~s}^{-1} \\
(7)\end{array}$ & $\begin{array}{l}\bar{V}_{b} \\
\mathrm{~km} \mathrm{~s}^{-1} \\
(8)\end{array}$ & $\begin{array}{l}\tau \\
\text { Myr } \\
(9)\end{array}$ & (10) & & $\begin{array}{l}n_{\mathrm{SN}} \\
(12)\end{array}$ & $\begin{array}{l}n_{\mathrm{SN}}^{\prime} \\
(13) \\
\end{array}$ & $\begin{array}{l}n_{\mathrm{SN}}^{\prime \prime} \\
(14)\end{array}$ & $\begin{array}{l}\text { Mst } \\
\left(M_{\odot}\right) \\
(15)\end{array}$ \\
\hline LCC & $285-312$ & $-10+21$ & 118 & $30-40$ & +4.2 & -1.6 & +1.4 & $11-12$ & 42 & 7 & 1.3 & $3 \pm 2$ & 6 & 1300 \\
\hline UCL & $312-343$ & $0+25$ & 140 & $50 \pm 20$ & +7.0 & -1.6 & +1.6 & $14-15$ & 66 & 17 & 3.5 & $6 \pm 3$ & 13 & 2100 \\
\hline US & $343-360$ & $+10+30$ & 145 & $50-60$ & +5.5 & -0.3 & -0.9 & $4-5$ & 49 & 15 & 0.8 & $1 \pm 1$ & 1 & 2350 \\
\hline
\end{tabular}

detected at $V \sim 0$ to $+5 \mathrm{~km} \mathrm{~s}^{-1}$, and identified as the undisturbed feature $A$. For the LCC-clump, which has no $V_{\mathrm{p}}$-value available due to blending effects, we considered several optical components of $V<0$, which were detected toward the clumps. For the faint branch $\mathrm{N}$, the results are somewhat contradictory, some nearby stars presenting IS components of the right velocities, and other ones not.

The results are shown in Table 2, Cols. 6-8, with the following meanings: 6) the upper limits derived for the distances $d$ of the near faces of the features having data available (For the undetected $\mathrm{M}_{1}-\mathrm{M}_{2}$ we derived a lower limit); 7) the upper limits derived for the near face of feature A with its velocity defined by P\&G's fit (e.g. Fig. 8); 8) the panel of Table A.1 and Fig. A.1. As can be seen, the upper limits derived in Col. 6 are consistent with the values obtained for $\bar{d}_{V 12}$ and $\bar{d}_{L 12}$ in Cols. 4 and 5 from the modeled $\mathrm{Na}$ I distributions. Moreover, the upper limits for $d_{\mathrm{A}}$ in Col. 7 are consistent with the distances fitted by P\&G in Fig. 9 taking due account of the thickness $\Delta d_{R}$ of feature A.

\section{The shells surrounding the Sco-Cen association}

A comparison of Figs. 1-4 shows that the pairs of branches $\mathrm{A}_{2}^{\prime} \& \mathrm{M}_{2}$ and $\mathrm{B}_{2} \& \mathrm{M}_{1}$ have similar positions and curvatures. However, in Fig. 6 these pairs show no continuity in $V_{\mathrm{p}}$, suggesting sheetlike features with an approaching edge, and a receding one, as is characteristic of shells.

In this section we analyze which of the HI features listed in Table 2 could be fitted by expanding shells. Like de Geus (1992) we fitted one shell for each stellar subgroup by means of Cappa de Nicolau \& Pöppel's (1986, from here on CN\&P) simple spherical model. It has the following parameters: i) the coordinates $l_{\mathrm{o}}, b_{\mathrm{o}}$ of the expansion center $\mathrm{O}$; ii) the angular size $2 \theta$; 

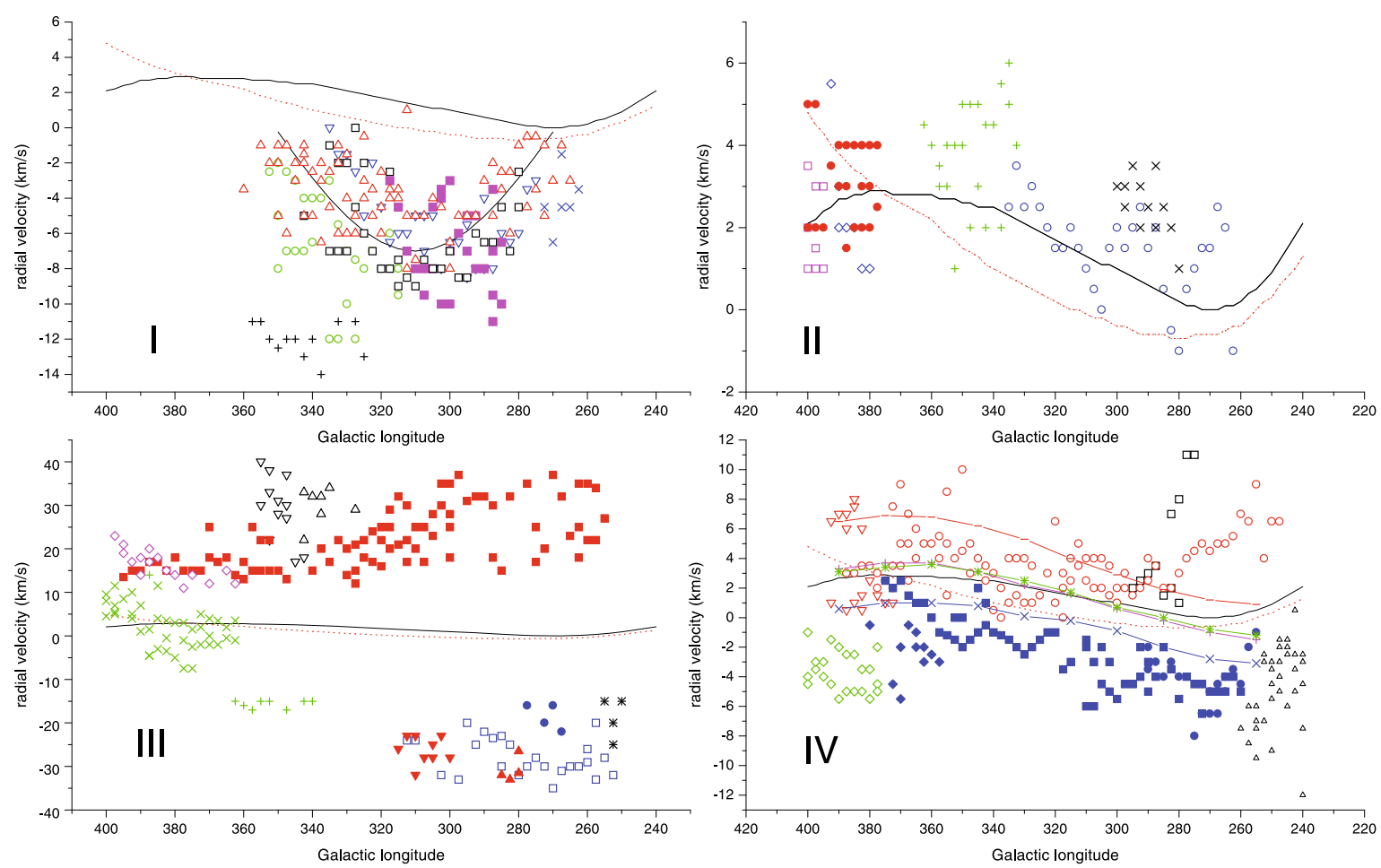

Fig. 6. Plot of the radial peak velocities $V_{\mathrm{p}}$ of the features defined in Figs. 1-4, with the following symbol meanings: Panel I: red triangles up, $\mathrm{B}_{1,2}$; black open squares, B'; green circles, B"; blue triangles down, C; blue $\times, \mathrm{C}_{\mathrm{t}}$; magenta solid squares, LCC-branch; +, SvW cloud (see Sect. 5). Panel II: red solid circles, HI toward Aql Rift; blue diamonds, $\mathrm{A}^{+}$; blue open circles, unshifted H I ridge north of branch C; $\times$, Cha arc; magenta open squares, $\mathrm{L}_{1}^{\prime}$; green +, US-loop. Panel III: red solid triangles down, $\mathrm{C}^{\prime}$; red solid triangles up, $\mathrm{C}^{\prime \prime}$; magenta diamonds, $\mathrm{D}_{0}^{\prime}$; green $\times, \mathrm{E}$; green ,$+ \mathrm{E}^{\prime}$; blue solid circles, J; blue open squares, $\mathrm{K} ;{ }^{*}, \mathrm{~L}_{2}^{\prime}$; red solid squares, $\mathrm{M}_{1,2} \& \mathrm{~N}$; black open triangles down, P1; black open triangles up, P2. Panel IV: blue solid squares, $\mathrm{A}_{1,2}$; blue solid diamonds, $\mathrm{A}_{1}^{\prime}$; blue solid circles, $\mathrm{A}_{2}^{\prime}$; red triangles down, $\mathrm{A}^{++}$; red open circles, $\mathrm{D}_{0,1,2,3}$; black open squares, $\mathrm{D}_{\mathrm{t}}$; green open diamonds, $\mathrm{L}_{1}$; black triangles up, $\mathrm{L}_{2}$. In all the panels $\mathrm{P} \& \mathrm{G}$ 's fit and Olano's model 2 are shown as a continuous black line and a dotted red line, respectively. In panel I the lower continuous curve corresponds to $V_{0} \cos [\theta \times(1+d / R)]$ with $V_{0}=6 \mathrm{~km} \mathrm{~s}^{-1}, \theta=l-310^{\circ}$, and $d / R=1.2$. In panel IV the four dashed curves correspond to the mean velocities of P\&M's test points, derived for $b=-35^{\circ}\left(\right.$ red - ); $-45^{\circ}$ $($ green $*) ;+35^{\circ}$ (magenta + ); and $+45^{\circ}$ (blue $\left.\times\right)$, respectively (see Sect. 7). The four panels are considered in Sect. 6.2.

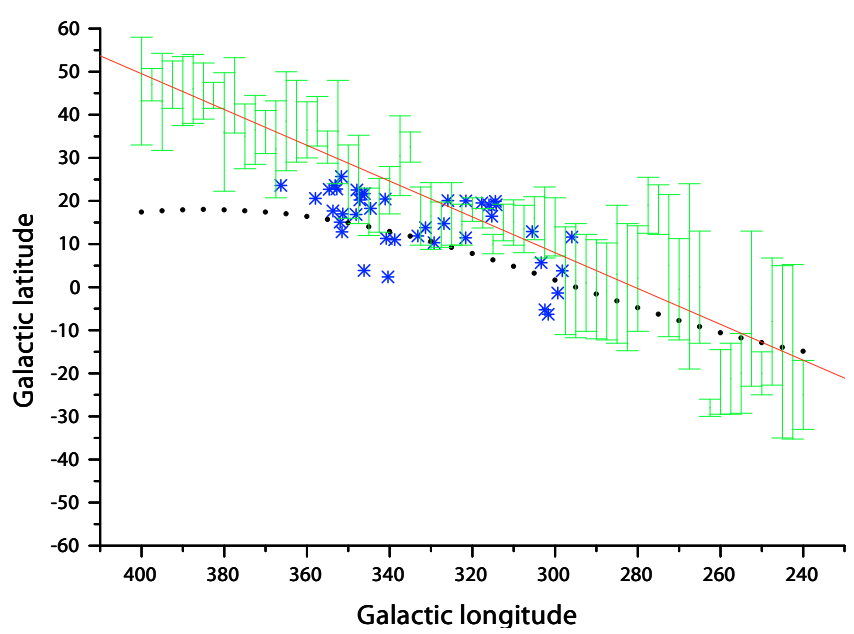

Fig. 7. Schematic plot of the $l-b$ stripe containing the systematic velocity shifts of the H I ridge. The vertical green bars indicate the selected widths $\Delta b$. The thin red line is a linear fit of their centers. The blue stars indicate members of the Sco-Cen association, and the black dots the plane of the GB (see the references of Fig. 1 and the text for more details.)

iii) the average radial velocity $V_{\mathrm{s}}$ of the substratum, (we adopted the velocity $V_{R}\left(b_{1}\right)$ of the originally undisturbed $G B$ gas at $\mathrm{O}$; see Sect. 3.2); iv) the present expansion velocity $V_{\mathrm{o}}$, numbering the fits by $n$. The fitted parameters are shown in Table 3, Cols. 1-6.

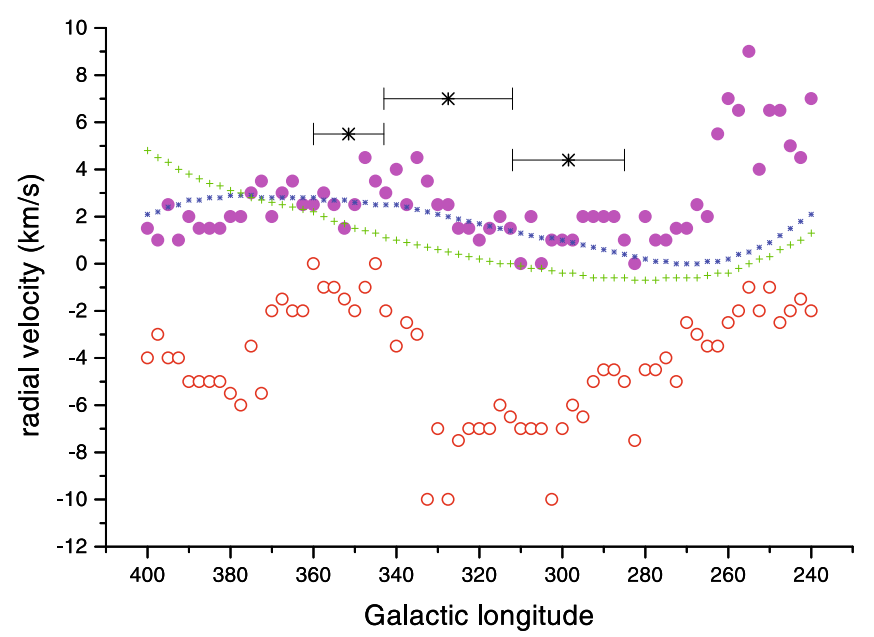

Fig. 8. Plot of the southern radial velocities $V_{R}\left(b_{1}\right)$ (solid magenta circles), and the northern ones $V_{R}\left(b_{2}\right)$ (open red circles) of the H I ridge at both ends of the stripe of velocity shifts, as a function of $l$. The large black stars correspond to the mean radial velocities of the stellar subgroups, the bars to their $l$-ranges (see Table 1). The small blue stars and green crosses correspond to P\&G's fits and to Olano's model 2, respectively. See the text for more details.

The distances $r_{\mathrm{o}}$ of the expansion centers were set equal to the distances of the corresponding stellar subgroups (see Table 1). The radius of the shell is $R=r_{\mathrm{o}} \times \sin \theta$. The largest 
W. G. L. Pöppel et al.: The interstellar medium surrounding the Scorpius-Centaurus association revisited

Table 2. Main characteristics derived for the H I features pointed out in Figs. 1-4. See the text for the details.

\begin{tabular}{|c|c|c|c|c|c|c|c|c|c|c|c|c|c|}
\hline $\begin{array}{l}\text { HI- } \\
\text { feature } \\
\text { (branch) } \\
\text { (1) }\end{array}$ & $\begin{array}{l}\bar{\Delta} \theta \\
\circ \\
(2)\end{array}$ & $\begin{array}{l}\bar{T}_{\mathrm{p}} \\
\mathrm{K} \\
(3)\end{array}$ & $\begin{array}{l}\mathrm{Na} \mathrm{I} \\
k_{V}, \bar{d}_{V 12} \\
\mathrm{pc} \\
(4) \\
\end{array}$ & $\begin{array}{l}\mathrm{NaI} \\
k_{L}, \bar{d}_{L 12} \\
\mathrm{pc} \\
(5) \\
\end{array}$ & $\begin{array}{l}d \\
\text { pc } \\
(6)\end{array}$ & $\begin{array}{l}d_{\mathrm{A}} \\
\mathrm{pc} \\
(7)\end{array}$ & $\begin{array}{l}\text { Panel } \\
\text { of } \\
\text { Table A.1 } \\
(8)\end{array}$ & $\begin{array}{l}\bar{d} \\
\mathrm{pc} \\
(9)\end{array}$ & $\begin{array}{l}\bar{\Delta} d \\
\mathrm{pc} \\
(10)\end{array}$ & $\begin{array}{l}\bar{\Delta} t \\
\mathrm{pc} \\
(11)\end{array}$ & $\begin{array}{l}N_{\mathrm{HI}} \\
10^{20} \\
\mathrm{~cm}^{-2} \\
(12) \\
\end{array}$ & $\begin{array}{l}n_{\mathrm{HI}} \\
\mathrm{cm}^{-3} \\
(13)\end{array}$ & $\begin{array}{l}\delta M_{\mathrm{HI}} \\
M_{\odot} \\
(14)\end{array}$ \\
\hline $\mathrm{A}_{1}$ & 6 & $29 \pm 10$ & $2,80-130$ & $3,80-145$ & $<99$ & & 1 & $80^{1}$ & 60 & 8 & 5.3 & 2.9 & 5000 \\
\hline $\mathrm{A}_{1}^{\prime}$ & 9 & $15 \pm 5$ & $1,80-130$ & & $<134$ & $<132$ & 2 & $80^{3}$ & 50 & 13 & 2.7 & 1.7 & 540 \\
\hline $\mathrm{A}_{2}$ & 6 & $16 \pm 6$ & $2,75-125$ & $2,75-130$ & $<80$ & & 3 & $80^{1}$ & 55 & 8 & 2.9 & 1.7 & 670 \\
\hline $\mathrm{A}_{2}^{\prime}$ & 6 & $18 \pm 7$ & $1,120-200$ & $2,125-200$ & - & & & $120^{2}$ & 75 & 13 & 3.3 & 1.4 & 1700 \\
\hline $\mathrm{B}_{1}^{2}$ & 6 & $19 \pm 5$ & $4,75-120$ & $4,70-160$ & $<150$ & $<150$ & 4 & $80^{2}$ & 70 & 8 & 3.5 & 1.6 & ) \\
\hline $\mathrm{B}_{2}$ & 7 & $16 \pm 7$ & $2,75-125$ & $2,130-215$ & - & & & $80^{3} ?$ & 75 & $10 ?$ & 2.9 & 1.3 & 4600 \\
\hline $\mathrm{B}^{\prime}$ & 5 & $16 \pm 8$ & $4,75-130$ & $4,80-155$ & - & & & $80^{2}$ & 65 & 7 & 2.9 & 1.4 & ) \\
\hline $\mathrm{B}^{\prime \prime}$ & 7 & $17 \pm 6$ & $2,75-130 ?$ & $2,40-80 ?$ & $<130$ & $<130$ & 5 & $75^{3} ?$ & 50 & $9 ?$ & 3.1 & 2.0 & 1800 \\
\hline $\mathrm{C}$ & 3 & $25 \pm 8$ & $3,160-220$ & $4,90-215$ & - & $<143$ & 6 & $100^{1}$ & 90 & 5 & 4.6 & 1.7 & 8300 \\
\hline$C^{\prime}, C^{\prime \prime}$ & 4 & $9 \pm 2$ & & & - & & & $\geq 200^{4}$ & & $\geq 14$ & 1.6 & & $\geq 280$ \\
\hline $\mathrm{J}, \mathrm{K}$ & 3 & $9 \pm 4$ & & & - & & & $100^{4} ?$ & & $5 ?$ & 1.6 & & 200 \\
\hline LCC-br. & 6 & $21 \pm 4$ & $2,75-130$ & $2,60-100 ?$ & $<143$ & & 7 & $70^{2}$ & 50 & 7 & 3.8 & 2.5 & 1400 \\
\hline LCC-cl. & & $25 \pm 6$ & & & $<120$ & & 7 & $70^{3} ?$ & & & 4.6 & & 730 \\
\hline $\mathrm{A}^{+}, \mathrm{A}^{++}$ & 6 & $18 \pm 3$ & $3,70-115$ & $3,75-165$ & - & & & $70^{2}$ & 70 & 7 & 3.3 & 1.5 & 800 \\
\hline $\mathrm{D}_{0}$ & 9 & $31 \pm 7$ & $3,100-150$ & $3,100-200$ & $<190$ & & 8 & $160^{1}$ & 75 & 25 & 5.7 & 2.5 & 9700 \\
\hline $\mathrm{D}_{0}^{\prime}$ & 6 & $8 \pm 3$ & & & $<288$ & & 8 & $160^{4} ?$ & $75 ?$ & & 1.5 & & 1300 \\
\hline $\mathrm{D}_{1}$ & 5 & $21 \pm 8$ & $4,160-215$ & $4,100-160$ & - & & & $130^{3}$ & 60 & 11 & 3.8 & 2.1 & 7800 \\
\hline $\mathrm{D}_{2}$ & 5 & $24 \pm 7$ & $2,120-180$ & $2,100-200$ & - & & & $110^{3}$ & 80 & 10 & 4.4 & 1.8 & 1400 \\
\hline$D_{t}$ & 4 & $20 \pm 5$ & $1,120-180 ?$ & & $<150$ & & 9 & $120^{3} ?$ & 60 & $8 ?$ & 3.7 & 2.0 & 1800 \\
\hline US-loop & 8 & $35 \pm 12$ & $3,75-130 ?$ & $5,50-130 ?$ & $<132$ & $<134$ & 10 & $140^{1}$ & 40 & 20 & 6.4 & 5.2 & 22000 \\
\hline Cha arc & 9 & $37 \pm 3$ & & $2,100-250 ?$ & $<175$ & & 11 & $100^{3} ?$ & $75 ?$ & $16 ?$ & 6.8 & $2.9 ?$ & 1500 \\
\hline $\mathrm{M}_{1}, \mathrm{M}_{2}$ & 4 & $5 \pm 2$ & & & $>278$ & & 16 & $280^{4} ?$ & & $>20$ & 0.9 & & 18000 \\
\hline $\mathrm{N}$ & 4 & $4 \pm 1$ & & & $<90 ?$ & & 17 & $90^{4} ?$ & & $6 ?$ & 0.7 & & 450 \\
\hline Aql Rift & & $>45$ & $1,70-200 ?$ & $2,50-250$ & - & $<145$ & 12 & & & & $>8.2$ & & \\
\hline $\mathrm{E}$ & 5 & $14 \pm 6$ & $2,105-155$ & $2,110-210$ & $<203$ & & 13 & $110^{2}$ & 75 & 10 & 2.6 & 1.1 & 3900 \\
\hline
\end{tabular}

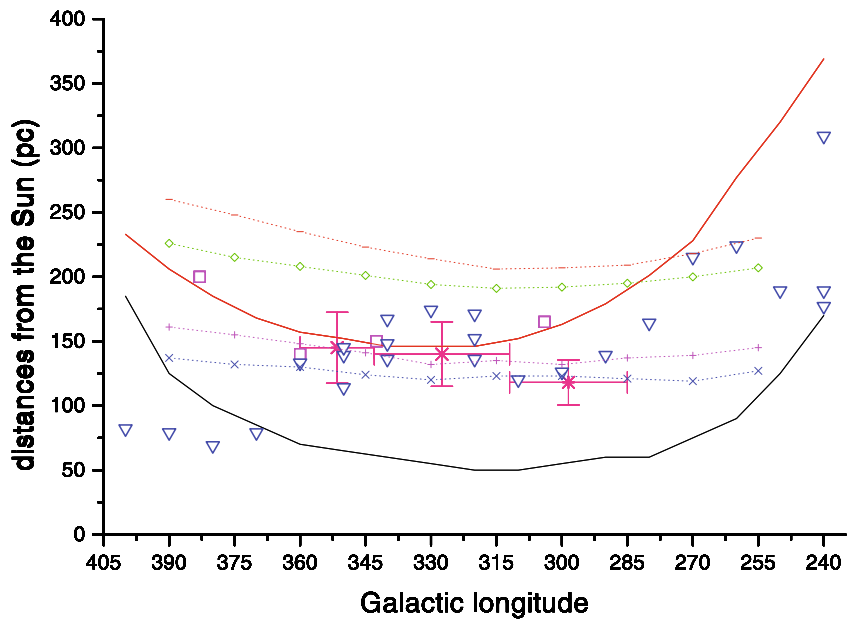

Fig. 9. Distances fitted by Lallement et al. (2003), to the absorption contours of $50 \mathrm{~mA}$ iso equivalent widths of the $D_{2}$-line along the Galactic plane (blue triangles down); mean distances of the stellar groups (red *, Table 1); mean distances of the molecular dark cloud complexes presumably associated with the GB (magenta squares, Table 4); distances of the expanding ring associated with the GB according to $\mathrm{P} \& \mathrm{G}$ 's fit (upper red curve) and Olano's model 2 (lower black curve). The four dotted curves correspond to the mean distances of P\&M's test points, derived for intermediate latitudes, namely $b=-35^{\circ}$ (red -$),-45^{\circ}$ (green diamonds), $+35^{\circ}$ (magenta + ), and $+45^{\circ}$ (blue $\times$ ), respectively (Sect. 7). See the text for more details.

approaching and receding radial velocities are expected in the direction of the center of the shell at the minimum and maximum distances to the observer, i.e. at $d_{\mathrm{MIN}} \simeq r_{\mathrm{O}}-R$ and $d_{\mathrm{MAX}} \simeq r_{\mathrm{O}}+R$, respectively. An observational upper limit $d_{\mathrm{OBS}}$ was derived for $d_{\text {MIN }}$ from optical IS lines, as explained below. The age was estimated very roughly as $\tau_{\mathrm{s}} \simeq R / V_{\mathrm{o}}$, since the average expansion velocity is unknown. For the young USloop, we used another procedure. Unlike de Geus (1992) we assumed that the US-loop is due to an SN (see Table 1), which occurred within the older US-shell (see Table 3, and Figs. 3 and 4). Since $R \ll r_{\mathrm{o}}$ we adopt CN\&P's snowplow model with $V(t) \cdot R^{j}(t)=$ Constant, $j=3$ (isotropy). The corresponding age is $\tau \simeq R(\tau) /(j+1) . V(\tau) \simeq 1 / 4\left(R / V_{\mathrm{o}}\right) \sim 0.8-1.0 \mathrm{Myr}$, which is consistent with de Geus' estimate of 1-1.5 Myr. The parameters mentioned above are shown in Table 3, Cols. 7-12.

In Figs. 10-15 we show the fits made for each shell on the central $V-b$ and $V-l$ contour maps. The H I features of Table 2 are indicated by arrows, including a nearby cloud discovered by Sancisi \& van Woerden (1970, from here on SvW, see CN\&P). For the sake of comparison, we plotted the fits of the velocities of the expanding ring according to both $\mathrm{P} \& \mathrm{G}$ and Olano, as well as the velocities of the nearby parts of the local arm or other local feature (OLF, see Sandqvist et al. 1976). The H I features fitted by the shells are listed in Table 3, Col. 13; additional non fitted but probable shell-components are signed with "?".

As can be seen, in each one of Figs. 10-15 the H I ridge shows two different regions, namely, one where its mean velocity $V_{R}$ is fitted approximately by P\&G's model, and the other one where $V_{R}$ is shifted by $\Delta V_{R} \sim-7$ to $-10 \mathrm{~km} \mathrm{~s}^{-1}$. The approaching hemispheres of the shells were fitted to the intense clumpy shifted ridge. In contrast, the receding hemispheres correspond to rather tenuous broad wings. In several cases, there are weak IV features, which suggest over-accelerated gas clumps from the shells. For the three shells there is also optical evidence of both approaching and receding caps, as we mention in the following.

i) For the UCL-shell we considered IS lines within the area $l=309^{\circ}-340^{\circ}, b=0^{\circ}$ to $+26^{\circ}$ occupied by the UCL 
Table 3. Fitted parameters and derived results using CN\&P's spherical model for the expanding shells defined by the H I features surrounding the Sco-Cen association.

\begin{tabular}{|c|c|c|c|c|c|c|c|c|c|c|c|c|}
\hline $\begin{array}{l}\text { Stellar } \\
\text { subgroup } \\
(1) \\
\end{array}$ & $\begin{array}{l}l_{\mathrm{o}}, b_{\mathrm{o}} \\
\left({ }^{\circ}\right) \\
(2) \\
\end{array}$ & (3) & $\begin{array}{l}V_{\mathrm{s}} \\
\mathrm{km} \mathrm{s}^{-1} \\
(4)\end{array}$ & (5) & $\begin{array}{l}V_{0} \\
\mathrm{~km} \mathrm{~s}^{-1} \\
(6)\end{array}$ & $\begin{array}{l}r_{\mathrm{o}} \\
(\mathrm{pc}) \\
(7)\end{array}$ & $\begin{array}{l}R \\
(\mathrm{pc}) \\
(8)\end{array}$ & $\begin{array}{l}d_{\mathrm{MIN}} \\
(\mathrm{pc}) \\
(9)\end{array}$ & $\begin{array}{l}d_{\mathrm{MAX}} \\
(\mathrm{pc}) \\
(10)\end{array}$ & $\begin{array}{l}d_{\mathrm{OBS}} \\
(\mathrm{pc}) \\
(11) \\
\end{array}$ & $\begin{array}{l}\tau_{\mathrm{s}} \\
\mathrm{Myr} \\
(12)\end{array}$ & $\begin{array}{l}\text { Fitted H I-features } \\
(13)\end{array}$ \\
\hline UCL & $\begin{array}{l}325 \pm 5 \\
17.5 \pm 5\end{array}$ & $\begin{array}{l}130 \\
\pm 10\end{array}$ & +3 & $\begin{array}{l}1 \\
2 \\
3\end{array}$ & $\begin{array}{l}8 \\
10 \\
12\end{array}$ & 140 & 127 & 13 & 267 & $<76$ & $\begin{array}{l}15.6 \\
12.7 \\
10.6\end{array}$ & $\begin{array}{l}\text { ) } \mathrm{A}_{1}, \mathrm{~A}_{1}^{\prime}, \mathrm{B}_{1}, \mathrm{~B}_{2}, \mathrm{~B}^{\prime}, \mathrm{B}^{\prime \prime}, \mathrm{C} \text {, } \\
\text { LCC-br., } \mathrm{M}_{1} \text { ?, } \mathrm{C}^{\prime} \text { ?, } \\
\text { ) nearby HI in GQ II at } V>0 \text { ? }\end{array}$ \\
\hline US & $\begin{array}{l}350 \pm 5 \\
21 \pm 4\end{array}$ & $\begin{array}{l}46 \\
\pm 6\end{array}$ & +3 & $\begin{array}{l}1 \\
2\end{array}$ & $\begin{array}{l}10 \\
12\end{array}$ & 145 & 57 & 88 & 202 & $<130$ & $\begin{array}{l}5.7 \\
4.8\end{array}$ & $\begin{array}{l}\text { ) } \mathrm{B}_{1}, \mathrm{~B}^{\prime \prime}, \mathrm{SvW} \text { cloud?, } \\
\text { ) US-loop }\end{array}$ \\
\hline $\begin{array}{l}\text { (US- } \\
\text { loop) }\end{array}$ & $\begin{array}{l}346 \pm 5 \\
25 \pm 5\end{array}$ & $\begin{array}{l}28 \\
\pm 4\end{array}$ & +3 & $\begin{array}{l}1 \mathrm{~b} \\
2 \mathrm{~b}\end{array}$ & $\begin{array}{l}8 \\
10\end{array}$ & 130 & 31 & 99 & 161 & & $\begin{array}{l}1.0 \\
0.8\end{array}$ & $\begin{array}{l}\text { US-loop } \\
\text { interior of US-loop = substr. }\end{array}$ \\
\hline LCC & $\begin{array}{l}300 \pm 5 \\
10 \pm 5\end{array}$ & $\begin{array}{l}90 \\
\pm 8\end{array}$ & +2 & $\begin{array}{l}1 \\
2 \\
3\end{array}$ & $\begin{array}{l}6 \\
9 \\
12\end{array}$ & 118 & 83 & 35 & 201 & $<60$ & $\begin{array}{l}13.8 \\
9.2 \\
6.9\end{array}$ & $\begin{array}{l}\text { ) LCC-branch and clump, } \mathrm{A}_{2} \\
\mathrm{~B}_{2}, \mathrm{~B}^{\prime}, \mathrm{M}_{1} \text { ?, } \mathrm{M}_{2} \text { ?, SvW cl.?, } \\
\mathrm{C} ?, \mathrm{Cha} \text { arc = substr. }\end{array}$ \\
\hline
\end{tabular}

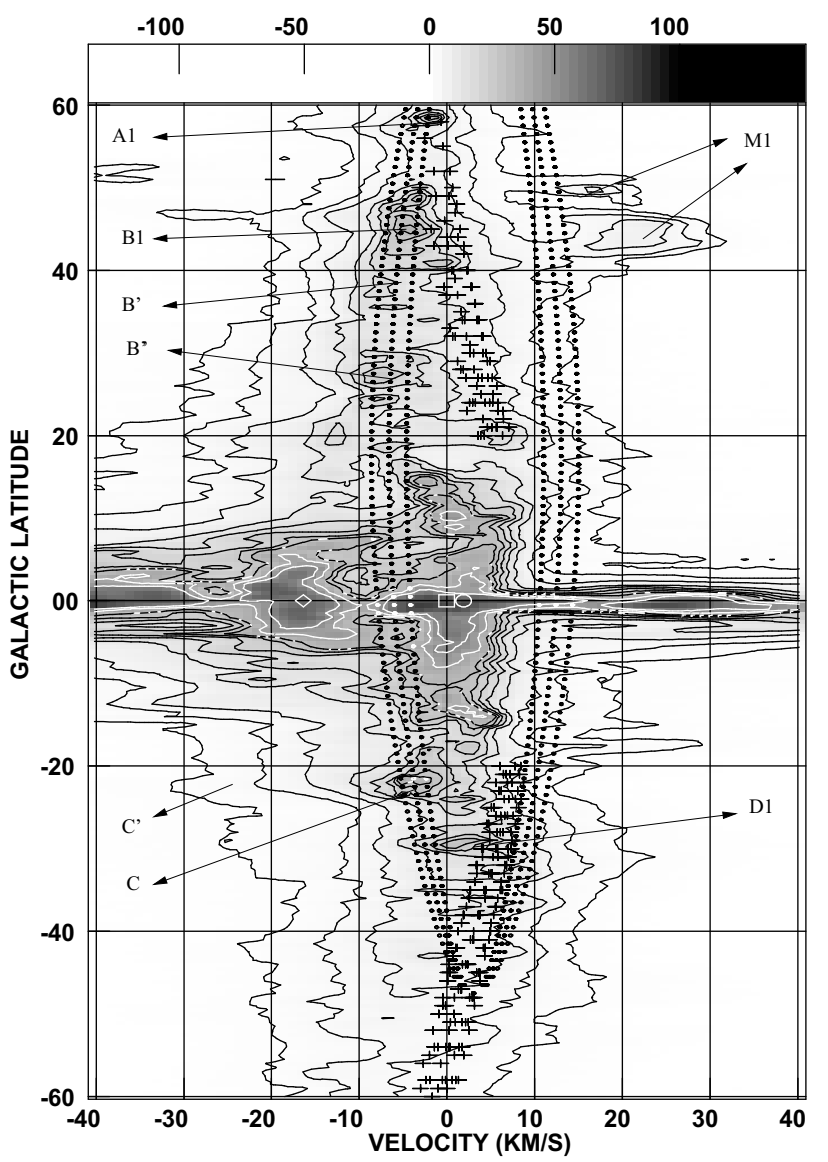

Fig. 10. Contour map for $l_{0}=325^{\circ}$. The levels are $1.48 \times(1,2,3.5$, $7,10.5,14,17.5,21,28$, and 35) $\mathrm{K}$. The selected $\mathrm{HI}$ features are indicated by arrows. The dotted curves indicate the fits of the UCL shell (see Table 3). At $b=0^{\circ}$, from left to right we also indicate the OLF (Sandqvist et al. 1976, white diamond), Olano's model 1 (white box) and P\&G's fit (white ellipse). The crosses correspond to P\&M's test points (see Sect. 7). See the text for the details.

subgroup (Table A.1, and Fig. A.1, panel 14). At $V<0$ the plot shows a group of warm and cold gas components at $d_{\max } \leq$ $76 \mathrm{pc}$, having a mean velocity $\bar{V}_{-}=-12.4 \pm 6.1 \mathrm{~km} \mathrm{~s}^{-1}$. They are consistent with an approaching cap of the UCL shell. At $V>0$, the plot only shows cold components. One of them at $V=+15.4 \mathrm{~km} \mathrm{~s}^{-1}$ is consistent with a receding cap since it was detected toward a star at $d=198 \mathrm{pc}$ (see Crawford 2000). The remaining components are located at $d_{\max }=128 \mathrm{pc}$ with a mean

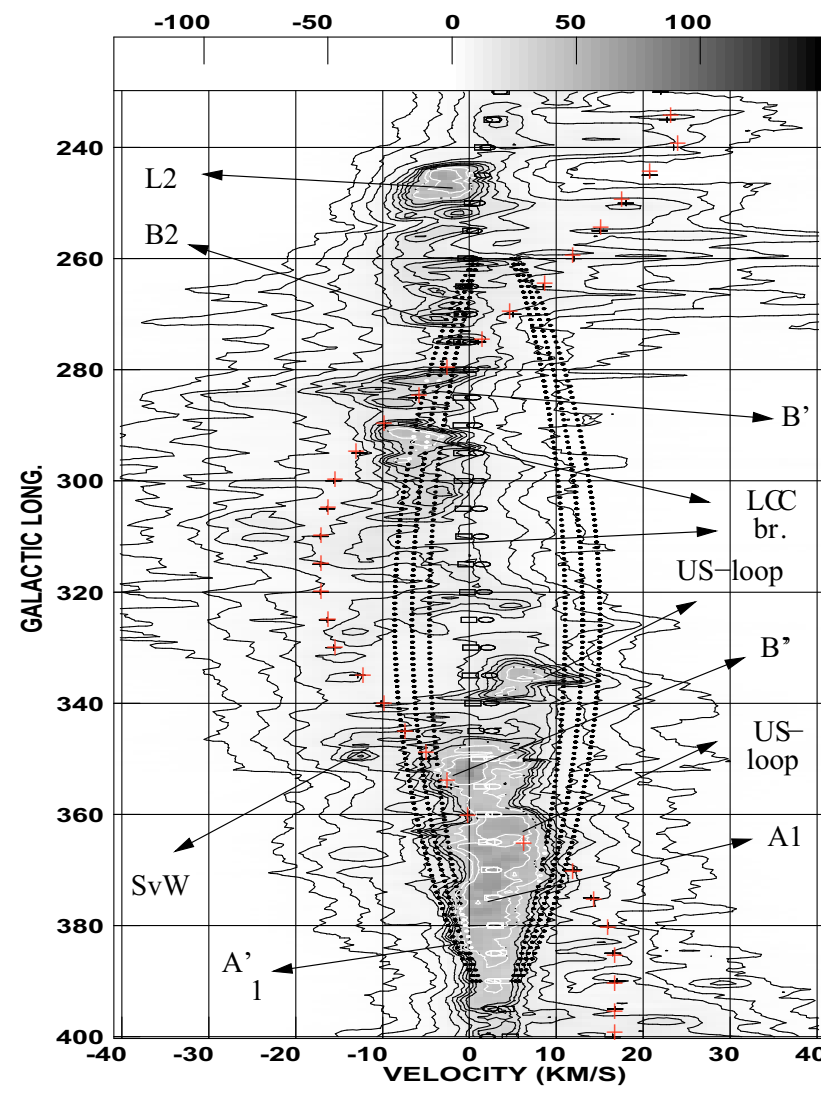

Fig. 11. Contour map for $b_{\mathrm{o}}=+17.5^{\circ}$. The levels are $1.48 \times(1,2$, $3.5,7,10.5,14,17.5,21,28$, and 35) K. The selected H I features are indicated by arrows. The dotted curves indicate the fits of the UCLshell (see Table 3). We also indicate the OLF (Sandqvist et al. 1976, red crosses), as well as P\&G's fit, and Olano's model 1 (boxes and ellipses, respectively). Both the latter are all near to $V \sim 0$, overlapping in some cases. See the text for the details.

velocity $\bar{V}_{+}=+6.0 \pm 1.4 \mathrm{~km} \mathrm{~s}^{-1}$. They should correspond to remnants of the substratum.

Actually, the momentum injected into the shell is not conserved since the input occurs pushwise, as is suggested by the number of $\mathrm{SN}$ explosions expected in the stellar subgroups (see Table 1). This is also consistent with the presence of IV branches like $C^{\prime}, C^{\prime \prime}, J$, and $K$, which should have a more recent origin; otherwise, they would have already overpassed the Sun during the age of the UCL subgroup. In addition, there should be significant inhomogeneities of the substratum. All this suggests the 
W. G. L. Pöppel et al.: The interstellar medium surrounding the Scorpius-Centaurus association revisited

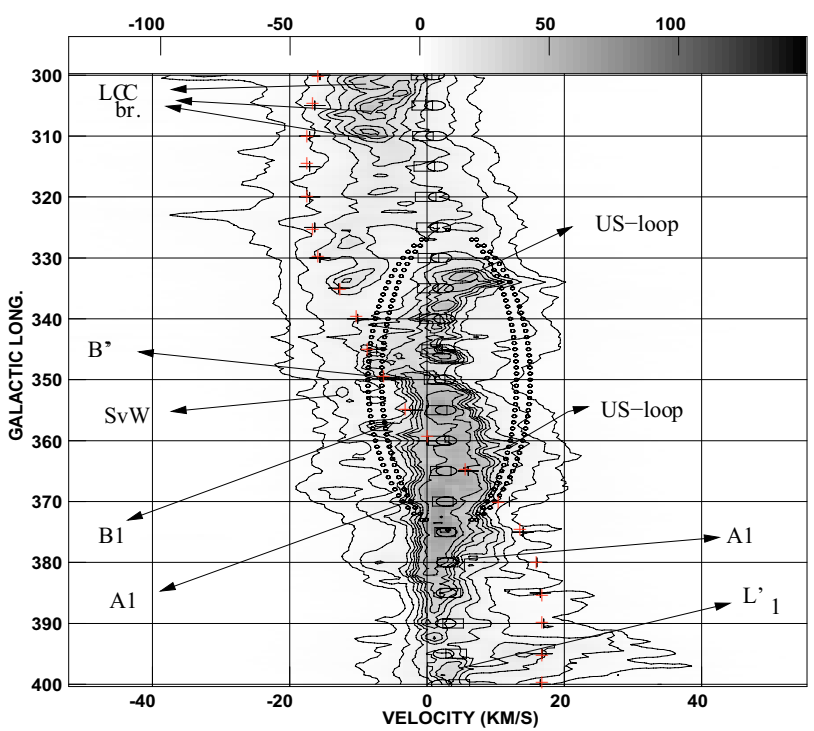

Fig. 12. Contour map for $b_{0}=+21^{\circ}$. The levels are $1.48 \times(2,4,8,12$, $16,20,24,32$, and 48$) \mathrm{K}$. The selected HI features are indicated by arrows. The dotted curves indicate the fits of the US-shell (see Table 3). We also indicate the OLF (Sandqvist et al. 1976, red crosses), as well as the P\&G fit, and Olano's model 1 (boxes and ellipses, respectively). Both of these are near to $V \sim 0$, overlapping in some cases. See the text for the details.

possibility of finding receding IS gas overshot into the GQ II at $V>0$. Such velocities are forbidden for objects in Galactic rotation. Table A.1, panel 18 shows a list of stars located in the GQ II at distances $d \leq 67 \mathrm{pc}$ with intense IS lines of Ca II or $\mathrm{Mg}$ II in the range $V=+7.4$ to $+18.7 \mathrm{~km} \mathrm{~s}^{-1}$ selected from the tables compiled by Redfield \& Linsky (2002, see for the details). If this IS warm gas originated at the UCL subgroup, its travel time into the GQ II should be shorter than the age of the subgroup. This should be expected for several of the clouds in view of their parameters $d$ and $V$ listed in Table A.1, as well as those of the UCL-subgroup in Table 1.

ii) For the US-shell we considered IS lines from the region $l, b \sim 343^{\circ}-354^{\circ},+16^{\circ}$ to $+33^{\circ}$. The plot shows many cold and warm IS components in the range $V \sim-21$ to $+11 \mathrm{~km} \mathrm{~s}^{-1}$, at $d_{\max } \sim 130$ pc (Table A.1, and Fig. A.1, panel 15). The components at $V<0$ suggest an approaching cap, with a mean velocity $V_{-}=-6.9 \pm 5.0 \mathrm{~km} \mathrm{~s}^{-1}$. The cap should include the portion of branch $\mathrm{B}^{\prime \prime}$ toward higher $l$, as well as the $\mathrm{SvW}$ cloud. For the latter we derived an updated upper boundary $d_{\max }=130 \mathrm{pc}$ for its distance (see Table A.1, panel 19).

The components at $V>0$ and $d_{\max } \sim 130$ pc should correspond to feature A ( $V \sim 0$ to $+5 \mathrm{~km} \mathrm{~s}^{-1}$, see Sect. 4.2), as well as to warm and cold gas accelerated by the extended H II regions S1, S7 and RCW129. These are all nearly enclosed by the US-shell with their exciting stars located at $d \sim 120-145 \mathrm{pc}$ (e.g. CN\&P). In addition, there is one cold component at $V \sim$ $+18 \mathrm{~km} \mathrm{~s}^{-1}$, as well as three warm ones at $V \sim+22,+30$, and $+43 \mathrm{~km} \mathrm{~s}^{-1}$, respectively. They were all detected toward stars located at $d \geq 200 \mathrm{pc}$, and should belong to the receding caps of either the US-shell or the UCL-shell. The broad wings, which are apparent in Fig. 13, could stem from these caps.

The assumption that the US-shell and the US-loop are two different expanding shells is consistent with CN\&P's Gaussian analysis of the cold HI in the vicinity of the US-subgroup. They found two main components, namely, $\mathrm{G}(V \sim-0.5$ to $\left.+2.5 \mathrm{~km} \mathrm{~s}^{-1}\right)$ and $\mathrm{P}\left(V \sim+3.5\right.$ to $+8 \mathrm{~km} \mathrm{~s}^{-1}$, see their Figs. 5

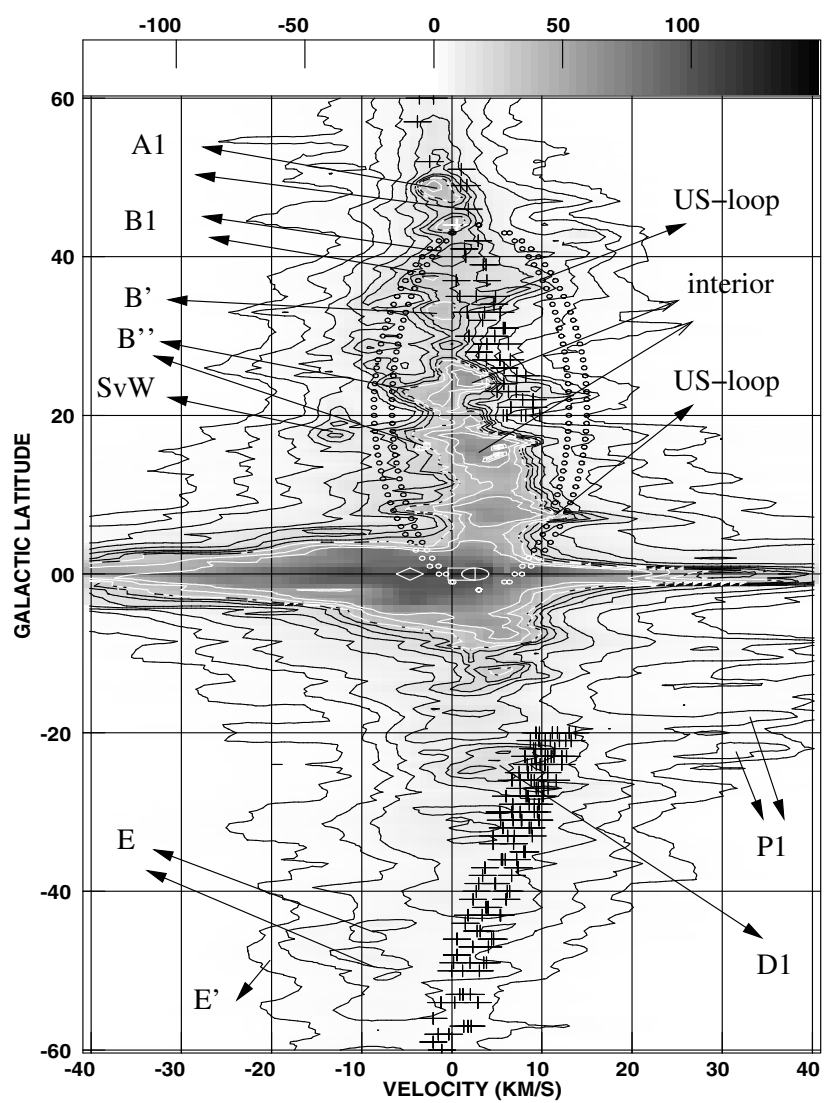

Fig. 13. Contour map for $l_{\mathrm{o}}=350^{\circ}$. The levels are $1.48 \times(1,2,3.5$, $7,10.5,14,17.5,21,28$, and 35) $\mathrm{K}$. The selected HI features are indicated by arrows. The dotted curves indicate the fits of the US-shell (see Table 3). At $b=0^{\circ}$, from left to right we also indicate: the OLF (Sandqvist et al. 1976, white diamond), Olano's model 1 (white box), and P\&G's fit (white ellipse). The crosses correspond to P\&M's test points (see Sect. 7). See the text for the details.

and 6). Component $\mathrm{G}$, which was identified with feature $\mathrm{A}$, has prominent clumps toward the interior of the US-loop. But an intense arclike region of $\mathrm{G}$ at $l>365^{\circ}$ was assigned to the edge of a shell. It should correspond to our fitted US-shell. Component $\mathrm{P}$, which was identified as an expanding shell, corresponds to the US-loop, to which it is adjacent at $l>350^{\circ}$. Actually, the distributions of both $\mathrm{P}$ and $\mathrm{G}$ have extensive gaps, which overlap partially at $b \geq+14^{\circ}$ with the filamentary Oph mdcc observed by de Geus et al. (1990) in CO (see their Figs. 3 and 4). This suggests that the Oph mdcc was swept up from the original substratum, first, partially by the US-shell and later its remaining bulk by the US-loop. The distances and the CO-velocities are consistent with this conclusion.

iii) For the LCC-shell, the right candidates for the approaching cap are the LCC-branch with the clump (see Fig. 2). Another right cap-candidate is the faint sheetlike IS structure detected by Corradi et al. (2004) southward of the LCC-branch at $d \leq 60 \mathrm{pc}$, with $\bar{V}=-7 \mathrm{~km} \mathrm{~s}^{-1}$, as mentioned in Sect. 4.1. In contrast, the Cha arc, which should correspond to the Mizuno et al. (2001) "filament A", appears to belong mainly to the original substratum (see Figs. 6, II, and 14). Moreover, the sequence of clumps in Fig. 15 correspond to the OLF and produces some blending with the GB gas at $l \sim 270^{\circ}-280^{\circ}$. 


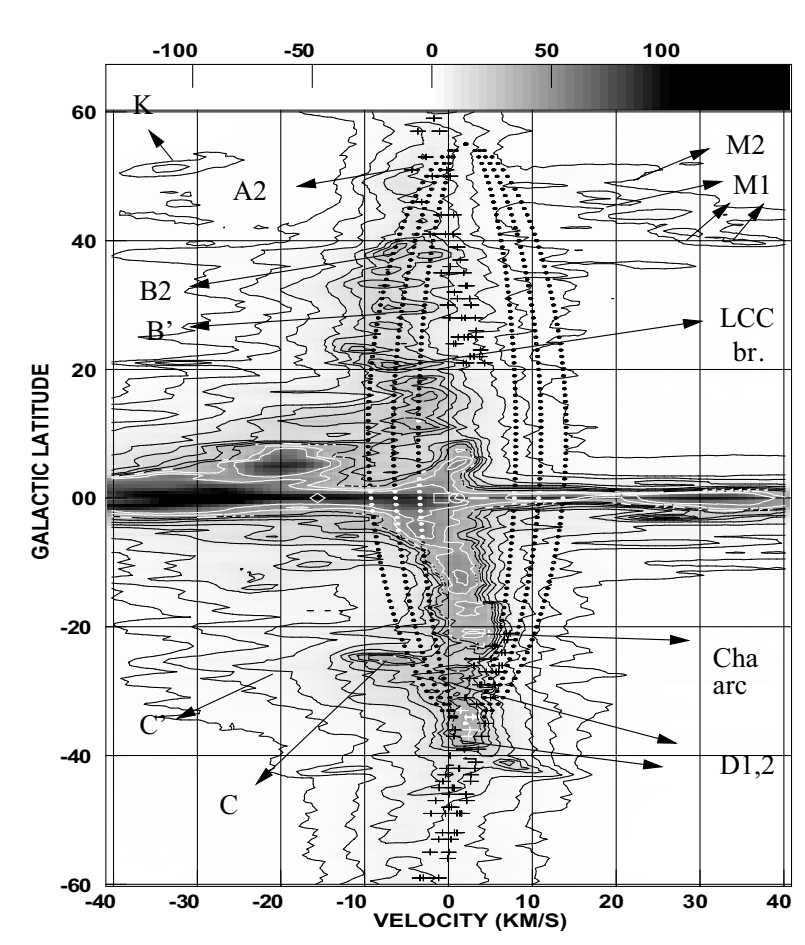

Fig. 14. Contour map for $l_{\mathrm{o}}=300^{\circ}$. The levels are $1.48 \times(1,2,3.5$, $7,10.5,14,17.5,21,28$, and 35) $\mathrm{K}$. The selected HI features are indicated by arrows. The dotted curves indicate the fits of the LCC-shell (see Table 3). At $b=0^{\circ}$, from left to right we also indicate: the OLF (Sandqvist et al. 1976, white diamond), Olano's model 1 (white box), and P\&G's fit (white ellipse). The crosses correspond to P\&M's test points (see Sect. 7). See the text for the details.

\section{Discussion of the results}

\subsection{Distances}

The results obtained in Cols. (4)-(6) of Table 2 show that most of the HI features of low velocity listed there are closeby. This conclusion was reinforced by the shell fits made in Sect. 5 . To estimate the distances of the H I features, we divided them into 4 groups. In group 1 are the features having measured distances $d$ available, namely, i) $\mathrm{A}_{1}-\mathrm{A}_{2}$, which comprise the H II region of $\alpha \operatorname{Vir}\left(l, b \sim 316^{\circ},+51^{\circ}\right)$, suggesting $d \sim 80 \mathrm{pc} \pm 7 \%$; ii) $D_{0}$, which should comprise the molecular cloud MBM 45 $\left(l=369.0^{\circ}, b=-28.0, V_{\mathrm{LSR}}=+6.7 \mathrm{~km} \mathrm{~s}^{-1}\right.$, Magnani et al. 1996 ) at a distance $d=160 \pm 15 \mathrm{pc}$ (Franco 1989); iii) the USloop, if we accept that it comprises the cloud Lupus 1, which has $V_{\mathrm{CO}}=0.17$ to $6.6 \mathrm{~km} \mathrm{~s}^{-1}$, and is located at $d=140 \mathrm{pc}$, whereas the radial thickness in the region is $\sim 40 \mathrm{pc}$ (Rizzo et al. 1998); and iv) C, for which Morras $(1980,1981)$ derived $d \sim 100$ pc. The features $\mathrm{A}_{1}-\mathrm{A}_{2}, \mathrm{C}$, and the US-loop were fitted by approaching shells in Sect. 5 . The results derived in Cols. (4)-(6) are consistent with these measured values of $d$. The group 2 contains the features having well consistent data in Cols. (4)-(6) of Table 2. The group 3 contains features, which have more uncertain values in Cols. (4)-(6), but were fitted by shells in Sect. 5.

For adopting the most reliable distances $\bar{d}$ and thicknesses $\bar{\Delta} d$ along the line of sight of the features in groups $1-3$, we considered either measured values, if available, or otherwise an evaluation of Cols. 4-6. The errors of $\bar{d}$ are estimated as $10-20 \%$ for

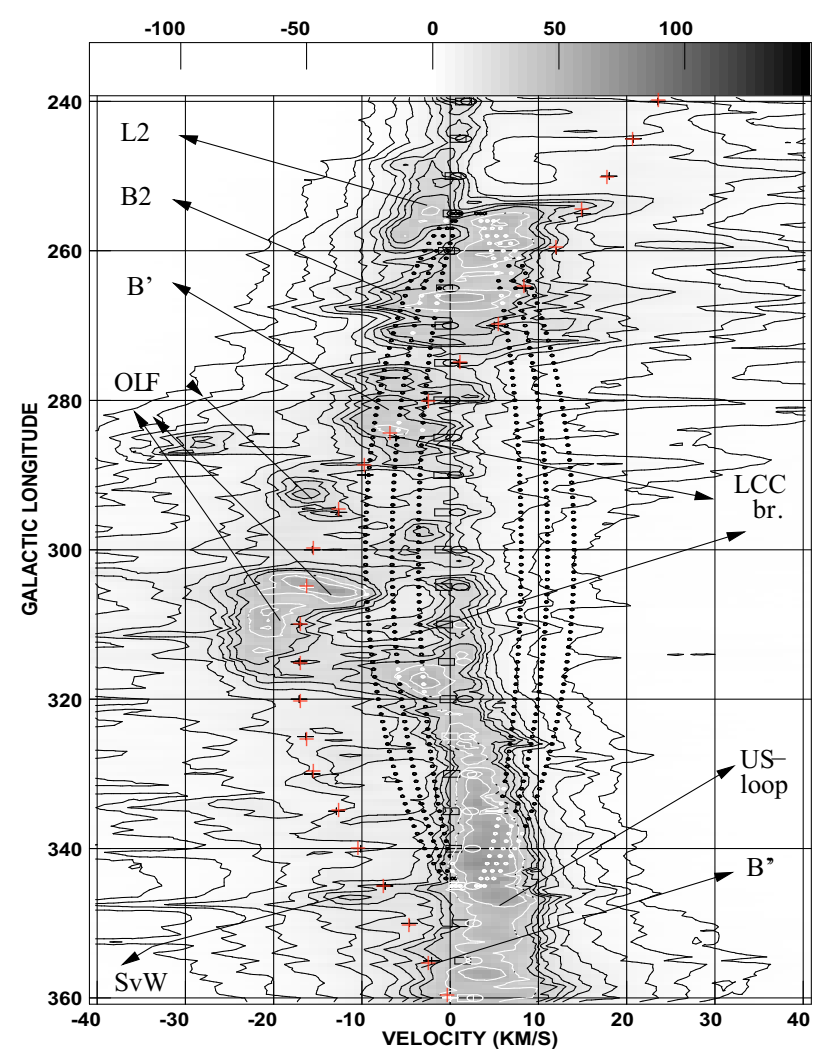

Fig. 15. Contour map for $b_{0}=+10^{\circ}$. The levels are $1.48 \times(1,2,3.5,7$, $10.5,14,17.5,21,28$, and 35) $\mathrm{K}$. The selected $\mathrm{HI}$ features are indicated by arrows. The dotted curves indicate the fits of the LCC-shell (see Table 3). We also indicate the OLF (Sandqvist et al. 1976, red crosses), as well as P\&G's fit, and Olano's model 1 (boxes and ellipses, respectively). Both of them are near to $V \sim 0$, overlapping in some cases. See the text for the details.

group 1, 20\%-30\% for group 2, and 30-50\%, or even larger for group 3. Moreover, there is group 4, which contains the features of IV. Their distances are very uncertain. For features $\mathrm{C}^{\prime}$ and $\mathrm{C}^{\prime \prime}$ Morras $(1980,1981)$ determined a minimum distance $\bar{d} \geq 200$ pc from polarization measurements. He showed a striking anticorrelation between these features and the gas of the expanding ring associated with the GB. This suggests that these branches stem from Lindblad's feature A. This should also be the case for branches $\mathrm{M}_{1,2}$ as suggested by their considerable angular extension (Figs. 3 and 4). Their high receding velocities (Figs. 10 and 14, as well as Fig. 6, panel III) could have moved them at several hundreds of parsecs from the Sun during the lifetime of both the older shells.

The results are shown in Cols. 9-14 of Table 2 with the following parameters: 9) the adopted distances $\bar{d}$ for the near faces with the assigned group as a superscript, and 10) the radial thicknesses $\bar{\Delta} d$ (values with uncertainties over $50 \%$ are marked with "?"); 11) the mean thickness across the line of sight $\bar{\Delta} t=\bar{\Delta} \theta \times \bar{d}$;12) the mean column density $N_{\mathrm{HI}}$, derived from $T_{\mathrm{p}}$ assuming low optical depths, and a Gaussian distribution with $\sigma_{V}=4 \mathrm{~km} \mathrm{~s}^{-1}$ (see Sect. 3.2); 13) the mean spatial density $\left.n_{\mathrm{HI}}=N_{\mathrm{HI}} / \bar{\Delta} d ; 14\right)$ the H I mass $\delta M_{\mathrm{HI}}$ of the feature obtained by numerical integration in Figs. 1-4 assuming low optical depth. In Eq. (1).

$\delta M_{\mathrm{HI}}\left(M_{\odot}\right)=11.45 \times 10^{-7} \cos \bar{b} \times(\bar{d}+0.5 \bar{\Delta} d)^{2} \times \Sigma T_{\mathrm{b}} \times S$,

$\Sigma T_{\mathrm{b}}$ is the sum of the brightness temperatures (in K) over the velocity channels involved, corrected for background, $\bar{b}$ the 
W. G. L. Pöppel et al.: The interstellar medium surrounding the Scorpius-Centaurus association revisited

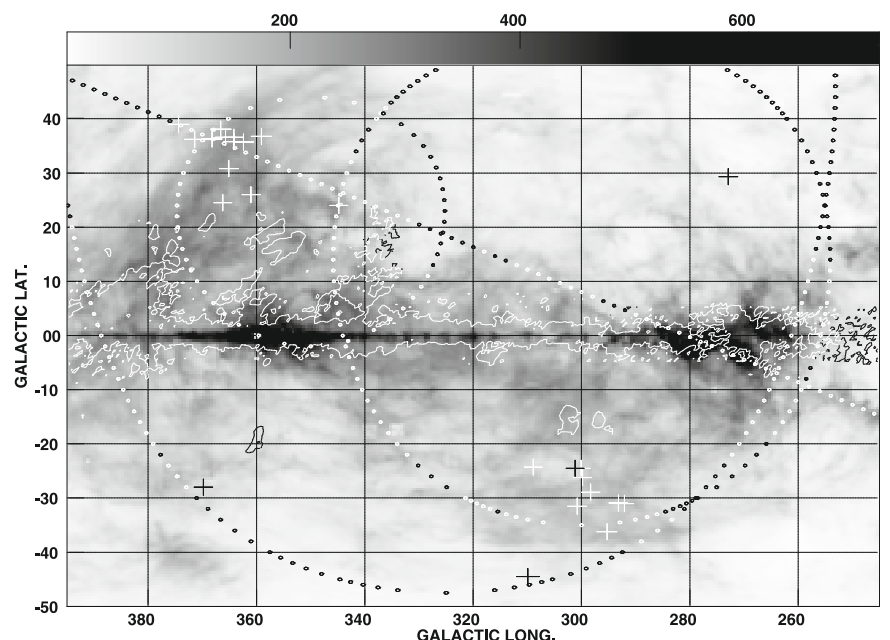

Fig. 16. Gray-scale map for HI with the channels integrated over $V=-1.0$ to $+3.1 \mathrm{~km} \mathrm{~s}^{-1}$, and a flux range $1-500 \mathrm{~K}$. Superposed are the contours of ${ }^{12} \mathrm{CO}(J=1-0)$ as derived from the survey by Dame et al. (2001). The contour level is $8.38 \times 0.30$. The crosses indicate molecular clouds of high latitude (see Hartmann et al. 1998; Magnani et al. 1996, 2000). The projections of the spherical shells fitted to each stellar subgroup are indicated by points, as well as the line fitted to the stripe of velocity shifts in Fig. 7. See the text for more details.

mean latitude, $S$ the area of the feature estimated in pixels of 0.25 sq. degr, and $(\bar{d}+0.5 \bar{\Delta} d)$ the middle distance of the feature. Given the uncertainties in $\bar{\Delta} d$, we used the average value of Col. 10 , i.e. $\langle\bar{\Delta} d\rangle=65 \pm 13$ pc (without considering values with "?") for all the features. The mean uncertainty of the H I masses is estimated at about $20 \%$ for the integration errors plus the errors of the squared middle distances.

The average of Col. 11 is $\langle\bar{\Delta} t\rangle=11 \pm 5$ pc without considering values with "?" or " $>$ ". $\langle\bar{\Delta} d\rangle$ and $\langle\bar{\Delta} t\rangle$ differ by a factor 6 . If we assume that the values of $\langle\bar{\Delta} d\rangle$ do not depend on the a priori densities assumed in the NaI models, the factor 6 suggests that most of the HI features have radially elongated structures, as should be expected for shelllike features. The range of the mean spatial densities is $\sim 1-5 \mathrm{HI}$ at. $\mathrm{cm}^{-3}$.

\subsection{Formation of a large expanding bubble}

Figure 16 is a rectangular $l-b$ map showing the distribution of the $\mathrm{HI}$ within the velocity interval -1 to $+3 \mathrm{~km} \mathrm{~s}^{-1}$, which is characteristic of the GB gas in the region considered here (e.g. P\&G). We superposed the straight line fitted to the stripe of velocity shifts in Fig. 7, as well as the projections of the spherical shells fitted to each stellar subgroup of the Sco-Cen association. If we exclude the region $|b| \leq 10^{\circ}$, which is blended by the intense Galactic background, we expect that the map should mostly show the distribution of the GB gas, as well as contributions from the edges of shells moving perpendicular to the line of sight. As can be seen, to the north of the stripe of velocity shifts, the GB gas is very tenuous, aside from the dense edge of the young US-shell, indicating that the gas originally there was swept up by both the oldest stellar subgroups. In contrast, to the south of the stripe there are large amounts of undisturbed GB gas.

Figure 16 and the parameters of Table 3 show that the different shells overlap, suggesting merging interactions between them. An example is the formation of the US subgroup triggered by the passage of the UCL-shell (e.g. Preibisch et al. 2002). In
Table 4. Main molecular dark cloud complexes located within the region studied in this paper. (See the text for the details)

\begin{tabular}{|c|c|c|c|c|}
\hline $\begin{array}{l}\text { mdcc } \\
\text { (1) }\end{array}$ & $\begin{array}{l}l\left(^{\circ}\right), \\
b\left(^{\circ}\right) \\
(2)\end{array}$ & $\begin{array}{l}\bar{V}_{\mathrm{LSR}} \\
\left(\mathrm{km} \mathrm{s}^{-1}\right) \\
(3)\end{array}$ & $\begin{array}{l}\bar{d} \\
(\mathrm{pc}) \\
(4)\end{array}$ & $\begin{array}{l}M_{\text {mol }} \\
\left(M_{\odot}\right) \\
(5) \\
\end{array}$ \\
\hline $\mathrm{Oph}^{1}$ & $\begin{array}{l}350-370 \\
+6 \text { to }+24\end{array}$ & -1 to +6 & $\begin{array}{l}140 \\
(80-200)\end{array}$ & 9000 \\
\hline Lup $^{2}$ & $\begin{array}{l}335-350 \\
+5 \text { to }+25\end{array}$ & -4.5 to +10 & $150 \pm 10$ & 17000 \\
\hline Cha-Mus ${ }^{3}$ & $\begin{array}{l}292-316 \\
-33 \text { to }-8\end{array}$ & +0.5 to +7 & $150-180$ & 8300 \\
\hline Aql Rift ${ }^{4}$ & $\begin{array}{l}376-394 \\
0 \text { to }+20\end{array}$ & +5 to +10 & 200 & 150000 \\
\hline Aql South & $\begin{array}{l}386-400 \\
-25 \text { to }-12\end{array}$ & 0 to +15 & $<220$ & 4000 \\
\hline
\end{tabular}

References. 1) de Geus et al. (1990); de Geus \& Burton (1991); 2) Tachihara et al. (2001); Magnani et al. (1996); Rizzo et al. (1998); 3) Mizuno et al. (2001); 4) Dame et al. (1987, 2001); 5) Kawamura et al. (1999).

our models the distance between both centers is $\Delta r \simeq 60 \mathrm{pc}$. For an age difference of $10 \mathrm{Myr}$ between both subgroups, the mean expansion velocity should be $\bar{V}_{\mathrm{o}} \sim 6 \mathrm{~km} \mathrm{~s}^{-1}$. The new expanding shell produced by the US subgroup, merged into the UCL-shell. The SvW cloud and part of branch $\mathrm{B}^{\prime \prime}$ should both stem from this process, whereas the outer branch $A_{1}$, whose curvature is similar to that of the US-loop, should be reaccelerated gas (see Figs. 2, 3). Combi et al. (1995) point out a spurlike nonthermal feature with a size of about $20^{\circ} \times 15^{\circ}$, which they interpret as an interaction between a shock front and a cloudy IS medium (ISM). The feature lies between branches $\mathrm{B}^{\prime \prime}$ and $\mathrm{B}_{1}$ and is consistent with our scenario.

The main merging interactions should have occurred between both the oldest shells. Figure 2 and the results of Table 3 make apparent that the branches $\mathrm{A}_{2}, \mathrm{~B}_{2}, \mathrm{~B}^{\prime}$, and $\mathrm{C}$, which were considered as signatures of the UCL-shell, have extensions and curvatures also suggesting a possible relation to the LCC subgroup, presumably through an interaction between both shells. Assuming an age difference of $3 \mathrm{Myr}$ between the UCL and LCC subgroups, about $3 \mathrm{Myr}$ for each shell to start, $\Delta r \sim 45 \mathrm{pc}$, and $\bar{V}_{\mathrm{o}} \sim 10 \mathrm{~km} \mathrm{~s}^{-1}$ for each shell (see Table 3), we should expect that a collision started between both shells about 7-8 Myr ago, heating, ionizing, and expanding the gas involved. The entire process produced the large hole seen in Fig. 3, and the merging of both shells into one large expanding bubble fed by forthcoming SN explosions (see Table 1), stellar winds, and expanding $\mathrm{H}$ II regions.

Because of the initial inhomogeneities of the substratum, as well as to the discontinuities and anisotropies introduced by the processes, which inflated the bubble, the latter has an irregular shape, which should differ somewhat from the fitted spherical shells. The boundary of the bubble is not necessarily smooth everywhere. Its northern edge or "top" should be at high b, where the gas density is low. At the edges of the bubble, its radial velocity appears similar to that of the substratum. Actually, as mentioned at the end of Sect. 3.2, the dense branches $D_{1,2}$, which are located northwards of the southern edges of both the fitted UCLand LCC-shells (see Figs. 10 and 14), are roughly consistent with $\mathrm{P} \& \mathrm{G}$ 's curve, therefore the branches $\mathrm{D}_{1,2}$ should conform the southern edge or "bottom" of the bubble at $l \sim 270^{\circ}-350^{\circ}$. Notice the several molecular clouds of high latitude northwards of branch $\mathrm{D}_{1,2}$ (see Fig. 16). At $l>350^{\circ}$ the southern edge 
should be formed by branch $D_{0}$, which is roughly fitted by $\mathrm{P} \& \mathrm{G}$ 's curve within $2 \mathrm{~km} \mathrm{~s}^{-1}$, aside from a few higher values due to intrinsic density inhomogeneities at the edges (see Fig. 6, IV).

In contrast, at $l<270^{\circ}$ the larger velocities of branch $\mathrm{D}_{3}$ are consistent with the receding face of a shell south of the Gum nebula (see Reynoso \& Dubner 1997). The small branch $\mathrm{C}_{\mathrm{t}}$ corresponds to the approaching face (see Figs. 2 and 6, I). Thus, neither $D_{3}$ nor $C_{t}$ should belong to the Sco-Cen association. Moreover, the small branch $\mathrm{D}_{\mathrm{t}}$, which shows no interaction with $\mathrm{D}_{2}$, points toward a small X-ray emitting compact cluster of PMSs of about $8 \mathrm{Myr}$ near $\eta$ Cha at $l, b \sim 292.4^{\circ},-21.7^{\circ}$, and a distance of about 97 pc (Mamajek et al. 2000). The cluster was related to the passage of the bubble of the Sco-Cen association. On the other hand, the H I of $V>0$ located northwards of branch $\mathrm{C}$ should be more distant undisturbed GB gas.

The similarities of the positions of the centers, of the radii and of the distances of both our fitted UCL-shell and the Loop I of the radio continuum (e.g. Berkhuijsen et al. 1971) suggest that the boundaries of the resultant bubble toward higher and lower $l$ should include the branches $\mathrm{L}_{1}^{\prime}, \mathrm{L}_{1}, \mathrm{~L}_{2}$, and $\mathrm{L}_{2}^{\prime}$ (see Figs. 1-3). The first one at $V>0$ (Fig. 6, II), should belong to feature A. $\mathrm{L}_{1}$ and $\mathrm{L}_{2}$, which were associated with the Loop I (e.g. Berkhuijsen et al. 1971; Heiles \& Jenkins 1976 and the references therein), have $V<0$, which is forbidden for nearby objects on differential Galactic rotation in the GQs I and III (see Fig. 6, IV). Actually, the Loop I should be the result of a later SN explosion in the UCL-subgroup. The resulting SNR expanded within the ionized interior of the bubble, meeting and ablating material from the dense Aql molecular complex (Frisch 1998). Thus, the interior of the shell heated to emit the intense X-radiation of the NPS, which produces the shadow of the H I feature pointed out by Egger \& Aschenbach (1995). We computed the HI masses of $\mathrm{L}_{1}^{\prime}, \mathrm{L}_{1}, \mathrm{~L}_{2}$, and $\mathrm{L}_{2}^{\prime}$ as $\sim 1500,600,1300$, and $7 M_{\odot}$, respectively, assuming a common middle distance $d=130 \mathrm{pc}$.

Somewhat puzzling are the isolated intense branches E, $\mathrm{E}^{\prime}$, as well as the receding features of IVs $\mathrm{D}_{0}^{\prime}$, and $\mathrm{P}_{1,2}$ (Fig. 6, III). By its location and characteristics $\mathrm{E}, \mathrm{E}^{\prime}$, and $\mathrm{D}_{0}^{\prime}$ should be disturbed gas of the GB ring. Branch E correlates with a hot spot in the polarization of the radiocontinuum (Cleary et al. 1979). In fact, there are two large elongated spots of polarization observed by Wolleben (2007, his Fig. 1) at $1.4 \mathrm{GHz}$ namely, a fringe extending approximately from $l, b \sim 330^{\circ},-37^{\circ}$ to $370^{\circ},-15^{\circ}$, about $10^{\circ}$ wide, and an adjacent nearly circular spot centered at $355^{\circ},-33^{\circ}$ with a diameter of $15^{\circ}$. which corresponds to an SNR (Testori et al. 2008). On the other hand, the gas of branches $\mathrm{A}^{++}$, and $\mathrm{A}^{+}$appears to consist of both undisturbed gas from the ring, and gas already accelerated by the stars of the association (see Fig. 6, II and IV).

In addition, Fig. 16 shows Dame et al.'s (2001) CO-contours. In the region we are considering there are five massive mdccs presumably related to the GB. Their main parameters are summarized in Table 4. The meaning of the columns is the following: 1) the name; 2) the extension in $l, b ; 3$ ) the interval of radial velocities for the bulk of the cloud; 4) the mean distance; 5) the molecular mass.

The mdccs in Oph, Lup, and Cha-Mus are located south of the stripe of velocity shifts, where there are large amounts of undisturbed $\mathrm{HI}$. Because the values of $n_{\mathrm{H}}$ in Table 2 are too low for producing new mdccs, we conclude that the three mdccs should be remnants from the dissipated parental molecular clouds, where the Sco-Cen association formed (e.g. Tachihara et al. 2001). Their total molecular mass is about $34000 M_{\odot}$. In contrast, the Aql Rift appears to be a very dense and massive boundary to the Sco-Cen bubble toward higher $l$. The Aql Rift could be connected to the neighboring Aql South (Kawamura et al. 1999). The H I associated with the Aql Rift should be the dense clumps seen toward the mdcc at $l \sim 370^{\circ}-398^{\circ}, b \sim 0^{\circ}$ to $+20^{\circ}$, at similar velocities (see Figs. 3, 6 II and Tables 2 and 4$)$.

\subsection{Some physical characteristics of the bubble}

The bubble subtends the range $l \sim 250^{\circ}-390^{\circ}$, thus its transverse diameter should be nearly $250 \mathrm{pc}$. Its approaching hemisphere includes the five selected branches $\mathrm{B}_{1,2}, \mathrm{~B}^{\prime}, \mathrm{B}^{\prime \prime}, \mathrm{C}$, and LCC (with clump) with their near faces at 70-100 pc. Their peak velocities were fitted by the curve $V=-7 \times$ $\cos \left(2.2\left(l-310^{\circ}\right)\right) \mathrm{km} \mathrm{s}^{-1}$, which qualitatively suggests a global expansion with a mean velocity of $\sim 7 \mathrm{~km} \mathrm{~s}^{-1}$ centered at $l=$ $310^{\circ}$ (see Fig. 6, I). These characteristics are not shared by branches $A_{1}-A_{1}^{\prime}, A_{2}-A_{2}^{\prime}$, which appear to be re- or overaccelerated gas (Fig. 6, IV).

The mean extension of the $\mathrm{HI}$ ridge in $b$ is not smaller than $\Delta b \sim 60^{\circ}$. For a mean distance of 125 pc, (see Fig. 9, and Table 2, Col. 7), this means that $H \geq 130$ pc. This confirms that, because of its doughnutlike shape, the ring of the GB-gas can also provide the substratum in the $z$-direction for the propagating shells.

Actually, excluding the region $|b| \leq 10^{\circ}$, the bulk of H I in Fig. 2 suggests an approaching hemisphere with an extension $\Delta l \times \Delta b \sim 102^{\circ} \times 65^{\circ}$, centered at $l, b \sim 318^{\circ} \pm 1^{\circ},+11^{\circ} \pm 2^{\circ}$, and tilted about $14^{\circ}$ to the Galactic plane. Assuming an elliptical shape, a rough estimation can be made of the total H I mass $M$ of the approaching hemisphere, $M \sim \pi / 4 \times m_{\mathrm{H}} \times \bar{N}_{\mathrm{HI}} \times \Delta l \times \Delta b \times \bar{d}^{2} \sim$ $26000 M_{\odot}$, where $\bar{d} \sim 80 \mathrm{pc}$ is the assumed middle distance, $m_{\mathrm{H}}$ the mass of the $\mathrm{H}$-atom, and the bars indicate average values over the ellipse. As expected, the value of $M$ is higher than the sum of the masses derived for the five branches in Table 2. If $\Delta \bar{V}_{R} \sim-6.6 \mathrm{~km} \mathrm{~s}^{-1}$ is the mean velocity increment of the H I ridge (see Sect. 3.2), the corresponding momentum $P$ will be $P \sim 145000 M_{\odot} \mathrm{km} \mathrm{s}^{-1}$. The approaching hemisphere and the extensive remnants from the substratum form the "wall" of IS material mentioned by several authors, when considering the Local Bubble (e.g. Frisch 1998; Breitschwerdt et al. 2000; Smith \& Cox 2001).

The receding hemisphere of the Sco-Cen bubble should include the gas of the broad wings at $V>0$ mentioned in Sect. 5 (see Figs. 10-15), and the branches of IV $\mathrm{M}_{1,2}$ and $\mathrm{N}$ (see Fig. 6, III), which build an arc, whose highest $b$ is reached near $l \sim 310^{\circ}$ (Fig. 4). They should be over-accelerated material related to the UCL subgroup. The receding hemisphere is much more tenuous and irregular than the approaching one. Nevertheless, its mass might be considerable due to branches $\mathrm{M}_{1,2}$.

The bubble should contain a hot plasma produced by the SN explosions. Most of the HI-features pointed out in Figs. 2 and 3 can be identified in silhouette against the X-ray emission in the maps of the $1 / 4 \mathrm{keV}$ band of the ROSAT survey (see Snowden et al.'s 1997 Fig. 2). In addition, some hot spots are seen toward the large H I hole seen in Fig. 3. The IV features $\mathrm{C}^{\prime}, \mathrm{C}^{\prime \prime}, \mathrm{J}, \mathrm{K}$, and $\mathrm{L}_{2}^{\prime}$ at $V<0$ (see Figs. 1, and 6, III) should be streamers of low mass over-accelerated by more recent injections of energy and momentum into the ISM surrounding the UCL and LCC stellar subgroups. South of branch J there is a hot spot at the $1 / 4 \mathrm{keV}$ band, which should point to an energetic event. At the $1 / 4 \mathrm{keV}$ band, the absorbing column density $N_{\mathrm{HX}}$ for which the optical depth $\tau=1$ is $\sim 10^{20}$ atoms $\mathrm{cm}^{-2}$. Since most branches of Table 2 have $N_{\mathrm{H}}>N_{\mathrm{HX}}$, the $1 / 4 \mathrm{keV}$ radiation in the direction of the bubble stems mainly from its interior. 
W. G. L. Pöppel et al.: The interstellar medium surrounding the Scorpius-Centaurus association revisited

In contrast, at the $3 / 4 \mathrm{keV}$ band the radiation does not anticorrelate with the $\mathrm{HI}$ features, aside from the thick $H I$ toward the Aql Rift and the one embedding the Oph mdcc. This is consistent with the value $N_{\mathrm{HX}} \sim 1.9 \times 10^{21}$ atoms $\mathrm{cm}^{-2}$, derived by assuming assuming a $-8 / 3$ power dependence for the cross section of absorption (Snowden et al. 1997). The thick H I is opaque even to the radiation of the $3 / 2 \mathrm{keV}$ band (see Fig. 3 and Snowden et al.'s 1997 Figs. 3, and 4). Including $\mathrm{H}_{2}$ this means $N_{\mathrm{H}} \geq N_{\mathrm{HX}} \sim 5 \times 10^{21}$ atoms $\mathrm{cm}^{-2}$. Hot receding plasma should also be found outside of the Sco-Cen bubble, corresponding to escaping hot gas, originated in the SN explosions. We quote that the presence of hot gas related to the bubble is consistent with the $\mathrm{O}$ VI absorption wings at high positive velocity detected at the six positions sampled in this region by Sembach et al. (2003, see Fig. 3). These wings have no H I counterpart.

The expanding bubble should be surrounded by a shock wave, as is consistent with the stripe of velocity shifts (we refer to Sect. 3.2). The shocked gas to the north of the stripe cooled again, as indicated by the similar velocity widths of the $\mathrm{HI}$ ridge at positive and negative latitudes. Thus, the shock can be considered as isothermal. Its mean radial velocity $u_{\mathrm{r}}$ should be not lower than the mean velocity shift $\Delta \bar{V}_{R}=-6.6 \pm 2.4 \mathrm{~km} \mathrm{~s}^{-1}$ found in Sect. 3.2. Thus, $u_{\mathrm{r}} \geq 8 \times c$, where $c \sim 0.8 \mathrm{~km} \mathrm{~s}^{-1}$ is the isothermal sound velocity, assuming a temperature $T \sim 80 \mathrm{~K}$ for the cold gas. Another similar shock wave should be associated with the velocity shifts of branch $\mathrm{C}$.

On the other hand, the maps of optical polarization suggest that the largest HI branches of Table 2 are channeling the IS magnetic field (see Heiles \& Jenkins 1976). They look like "striations" (Weaver 1979) with longitudes up to about $150 \mathrm{pc}$, and they should have formed from elongated preexisting structures in the expanding GB ring of gas. The ratio of the mechanical specific energy to the magnetic one is $\rho \sim 1.06$ $n_{\mathrm{H}}\left(\mathrm{cm}^{-3}\right) V_{0}^{2}\left(\mathrm{~km} \mathrm{~s}^{-1}\right) / B^{2}(\mu \mathrm{G})$. Assuming $n_{\mathrm{H}} \sim 1.5 \mathrm{~cm}^{-3}, V_{0} \sim$ $10 \mathrm{~km} \mathrm{~s}^{-1}$ and values of $B$ of a few $\mu \mathrm{G}$, as are usually observed on a large scale in the IS medium, we obtain $\rho \gg 1$. Thus, on the scale of the branches, the gas dynamics drives the magnetic field. Obviously, the distribution of the magnetic field lines (see Cleary et al.'s Fig. 9) cannot be fitted by a dipolar distribution in the region considered here.

In the previous sections we have seen that most of the $\mathrm{H} \mathrm{I}$ in the region we are considering is local, as inferred from its kinematics and its distribution, which is clearly asymmetric to the Galactic plane in Figs. 1-4, aside from the stripe $|b| \leq 10^{\circ}$. The total H I mass $M_{\mathrm{HI}}$ embedding the Sco-Cen association was derived very rougly by integrating the lowest contour in each figure. We assumed low optical depths and subtracted a mean "absolute" background by averaging several positions surrounding the lowest contour. For the region $|b|<10^{\circ}$, the mass was linearly interpolated between both the stripes $|b|=10^{\circ}$ to $30^{\circ}$. We assumed middle distances $\bar{d}^{\prime}=100 \mathrm{pc}$ for Figs. 1 and 2, and $\bar{d}^{\prime}=150$ pc for Figs. 3 and 4 (see Table 2, and Fig. 9).

The results are shown in Table 5. The meaning of the columns is the following: 1) the figure considered; 2) the corresponding velocity range; 3 ) the assumed middle distance; 4) the mass computed for the ranges $b=-50^{\circ}$ to $-10^{\circ}$, and $+10^{\circ}$ to $+60^{\circ}$, with cōs $b \sim 0.83$ for Figs. $1-4 ; l=240^{\circ}-390^{\circ}$, for Figs. 1 and 2 , and $l=250^{\circ}-390^{\circ}$, for Figs. 3 and 4 , but excluding the region of the Gum nebula at $l<270^{\circ},|b|<20^{\circ}$; 5) the interpolated mass for the region $|b|<10^{\circ}$, and for the excluded Gum region in Figs. 3 and 4; 6) the resulting total H I mass.

According to these computations, the total H I mass embedding the Sco-Cen association is $M_{\mathrm{HI}} \sim 249000 M_{\odot}$, with a relative error similar to those of the masses in Table 2 . The mass of
Table 5. Total H I-masses of the ISM embedding the Sco-Cen association. (See the text for the details)

\begin{tabular}{llllll}
\hline \hline Fig. & $\begin{array}{l}\Delta V \\
\left(\mathrm{~km} \mathrm{~s}^{-1}\right)\end{array}$ & $\begin{array}{l}\bar{d}^{\prime} \\
(\mathrm{pc})\end{array}$ & $\begin{array}{l}M_{\text {comp }} \\
\left(M_{\odot}\right)\end{array}$ & $\begin{array}{l}M_{\text {interp }} \\
\left(M_{\odot}\right)\end{array}$ & $\begin{array}{l}M_{\mathrm{HI}} \\
\left(M_{\odot}\right)\end{array}$ \\
$(1)$ & $(2)$ & $(3)$ & $(4)$ & $(5)$ & $(6)$ \\
\hline 1 & -42 to -20 & 100 & 1550 & 650 & 2200 \\
2 & -20 to -0.5 & 100 & 43000 & 17000 & 60000 \\
\hline 3 & -0.5 to +16 & 150 & 115000 & 50000 & 165000 \\
4 & +16 to +41 & 150 & 15000 & 7000 & 22000 \\
\hline
\end{tabular}

all the features of Table 2 is $\Delta M_{\mathrm{HI}}^{\prime} \sim 94000 M_{\odot}$. This should be considered a lower limit because of the $d_{\min }$ obtained for branches $\mathrm{M}_{1,2}$. Including $3400 M_{\odot}$ from $\mathrm{L}_{1}^{\prime}, \mathrm{L}_{1}$, and $\mathrm{L}_{2}$, and assuming that all this HI stems from the GB, this suggests that not less than about $39 \%$ of the embedding H I was significantly disturbed (shocked) by the evolution of the stars of the Sco-Cen association. Including the molecular mass and $30 \%$ of $\mathrm{He}$, the total embedding mass is estimated at $M_{\mathrm{t}} \sim 368000 M_{\odot}$. Thus, the mass of the association (see Table 1 ) is at most $\sim 4.5 \%$ of the disturbed mass (including $\mathrm{He}$ ) and not more than $1.6 \%$ of the embedding mass. However, it is necessary to point out that all these numbers are severely affected by the uncertainties of the distances.

\subsection{Scenario}

We checked a scenario for the formation and evolution of the bubble surrounding the Sco-Cen association in terms of the large expanding ring of gas associated with the $G B$, as is consistent with our conclusions above. We used P\&M's computations of ballistic orbits of test particles, which assume such an explosive origin, and ignore the braking forces of the ISM. According to $\mathrm{P} \& \mathrm{M}$, this can be nearly justified for $|b| \geq 35^{\circ}$. We refer to their paper for the details.

As a first test of the scenario, Figs. 10, 13 and 14 show the radial velocities $V_{\mathrm{PM}}(b)$ of P\&M's test particles for $|b| \geq 20^{\circ}$. To improve the statistics we doubled the number of the test particles by interpolatig their initial velocities. As can be seen, in the three figures $V_{\mathrm{PM}}$ exceeds the values of $\bar{V}_{R}$ at $|b|=20^{\circ}$. The difference decreases with increasing $|b|$. For $b \leq-35^{\circ}$, where the $\mathrm{HI}$ ridge is undisturbed, $V_{\mathrm{PM}} \sim \bar{V}_{R}$, aside from the region $b \leq-45^{\circ}$ in Fig. 13, which corresponds to the disturbed branch $\mathrm{E}$ at more than $60^{\circ}$ from the GB circle. On the other hand, for $b \geq+35^{\circ}$ the values of $\bar{V}_{R}$ are shifted by $\Delta \bar{V}_{R} \sim-6.6 \mathrm{~km} \mathrm{~s}^{-1}$ (see Sect. 3.2, Fig. 8), whereas the predicted values $V_{\mathrm{PM}}$ are similar to the unshifted values of $\bar{V}_{R}$ at $b<0$. This is consistent with our interpretation of $\Delta V_{R}$ as disturbances of the $\mathrm{HI}$ of the GB produced by the stellar association (Sect. 4.1).

As a second test of the scenario, we computed the mean velocities $\bar{V}_{\mathrm{PM}}$ and distances $\bar{d}_{\mathrm{PM}}$ of the test particles in 40 cells of dimensions $\Delta l \times \Delta b=10^{\circ} \times 10^{\circ}$ centered at $l=$ $255^{\circ}, 270^{\circ}, \ldots, 390^{\circ}, b= \pm 35^{\circ}, \pm 45^{\circ}$, respectively. The mean number of test particles within each cell was $82 \pm 43$.

The results for $\bar{V}_{\mathrm{PM}}$ are plotted in Fig. 6, IV with intrinsic mean dispersions $\bar{\Delta} V_{\mathrm{PM}}=1.6 \pm 0.2 \mathrm{~km} \mathrm{~s}^{-1}$. As can be seen, for $l \sim 270^{\circ}$ to $380^{\circ}$ the values of $\bar{V}_{\mathrm{PM}}$ are similar to those of P\&G's fit within about $2 \mathrm{~km} \mathrm{~s}^{-1}$. In addition, at $l>280^{\circ}$ most of the peak velocities $V_{\mathrm{p}}$ of the southern branches $\mathrm{D}_{0,1,2}$ fall just between the curves of $\bar{V}_{\mathrm{PM}}$ for $b=-45^{\circ}$ and $-35^{\circ}$. This is consistent with the identification of branch $\mathrm{D}_{0,1,2}$ as the southern boundary of the bubble, which developed surrounding the ScoCen association and is seen edge-on (see Sect. 6.2). 
The results for $\bar{d}_{\mathrm{PM}}$ are plotted in Fig. 9 with intrinsic mean dispersions $\Delta d_{\mathrm{PM}}=29 \pm 3 \mathrm{pc}$. These are of the same order of magnitude as one half of the average thicknesses of the branches found at the end of Sect. 4.1. P\&G's fit for the distances falls just between the curves of $d_{\mathrm{PM}}$ for $b=-35^{\circ}$ and $-45^{\circ}$, and those for $b=+35^{\circ}$ and $+45^{\circ}$.

We conclude that, for $|b| \geq 35^{\circ}$, the P\&M model is consistent with the observations in the region under study, within the intrinsic uncertainties of the assumed parameters. It is consistent with the interpretation that the Sco-Cen association produced disturbances on the expanding ring of gas associated with the GB during its transit through it.

\section{Conclusions}

After pointing out the main $\mathrm{H}$ I features, as well as some smaller ones surrounding the Sco-Cen association in Sect. 3 (Figs. 1-5), we derived their kinematics (Fig. 6). Moreover, we showed that the expanding ring of $\mathrm{HI}$, which is associated with the GB, presents a narrow stripe of velocity-shifts of several $\mathrm{km} \mathrm{s}^{-1}$ on the celestial sphere (Fig. 7). To the south of the stripe P\&G's fit is consistent with the kinematics of the GB gas, whereas to the north of the stripe $V_{R}$ is systematically below P\&G's fit (Fig. 8). In Sect. 4.1 we compared our kinematical H I-maps with the maps of the spatial distribution of the IS Na I derived by Vergely et al. and Lallement et al. We concluded that the Sco-Cen association, which is located mainly to the north of the stripe, is just crossing the ring of gas (Fig. 9). Therefore, we interpreted the striking velocity shifts of the $\mathrm{HI}$ ridge, as a disturbance produced by the stars on the ring of gas. Complementarily, in Sect. 4.2 we derived distance ranges and upper limits from further optical data taken from the literature.

In Sect. 5 we fitted expanding spherical shells around each stellar subgroup, assuming that the original substratum was the GB gas (Figs. 10-15). In terms of the expanding velocities $V_{0}$, the shell ages are $\tau \sim 11-16$ Myr for the UCL-shell and 7-14 Myr for the LCC-shell. These ages are comparable to the ages of the subgroups, suggesting that the time needed by the association to stay within the medium should not have been smaller than $\tau$. Most of the HI features listed in Table 2 can be understood in terms of the fitted shells.

In Sect. 6.1, after evaluating what is known about the distances and radial thicknesses of the HI features, the most reliable values for $\bar{d}$ and $\bar{\Delta} d$ were adopted and the uncertainties estimated. As a consequence, we could derive some of the observational characteristics of the H I branches (Table 2).

In Sects. 6.2-3 we showed that the models suggest important interaction processes between the shells. These processes produced a combined large UCL-LCC-bubble, triggering the formation of the US-subgroup, as well as the observed low-mass star formation activity in the vicinity of the LCC-subgroup. The intense rim of the US shell at positive velocities should be the cause of the striking third peak observed in the $l-V$ relation for Lindblad's ring (e.g. Olano 1982; Sodroski et al. 1985, as well as Fig. 8). This peak is not predicted by the models of the ring. The mass of the approaching dense hemisphere of the bubble was estimated at $\sim 26000 M_{\odot}$, its distance at $\sim 80 \mathrm{pc}$, and its velocity at nearly $-6.6 \mathrm{~km} \mathrm{~s}^{-1}$. In contrast, the receding hemisphere is rather tenuous, apart from branches $\mathrm{M}_{1,2}$. Some local gas, at a peculiar $V>0$ within the GQ II, could be interpreted as overshot gas from the shells. The bubble, which should be surrounded by an isothermal shock-wave, contains hot gas, as is consistent with the ROSAT-data at the $1 / 4 \mathrm{keV}$ band.
Finally, in Sect. 6.4 we saw that for $|b| \geq 35^{\circ}$ the computational results derived from P\&M's model are consistent with the observational results found in Sects. 3 and 4, as well as with the assumption that the Sco-Cen association produced disturbances on the expanding ring of gas associated with the GB within the uncertainties intrinsic to the model.

What can be concluded about the origin of the Sco-Cen association? Since the ages of the fitted shells are consistent with the ages of both the older stellar subgroups, we expect that the stars of the association should have conserved the original velocities of the molecular cloud, where they formed. Actually, the tangential velocities of the models of the ring by Olano (1982) and by $\mathrm{P} \& \mathrm{G}$ have the opposite sense as those of the association (see Fernández 2005), and the Galactic rotation. However, from Lindblad's (2000) linear model of the GB as an inclined expanding and rotating disk, we should expect also a rotation of the associated ring of gas with its tangential velocities having the same sense as the Galactic rotation. This was confirmed by Bobylev (2006) for the GB. Therefore, the formation of the association should have been triggered during the accretion of the parental molecular cloud on the GB ring, and the place of formation should have been in the GQ I (e.g. Maíz-Apellániz (2001); Fernández 2005; et al. 2008).

Our results are consistent with Weaver's original basic scenario, as well as with de Geus' (1992) study. According to Mamajek et al. (2002) the gas associated with the Sco-Cen association should be part of a "spur" of H I and molecular clouds that runs from near LCC through Lupus and Ophiuchus and into the Aquila and Vulpecula Rifts. These should constitute the nearest reservoirs of IS gas for prosecuting the star formation process.

Acknowledgements. We thank Messrs. F. Bareilles, C. Cristina, and M. Fumagalli for computational help. In this research we made use of the NRAO Astronomical Image Processing System (AIPS). We also profited from the use of the SIMBAD database operated at the CDS, Strasbourg, France, as well as from the use of the NASA Astrophysics Data System (ADS). We used data files from available astronomical surveys produced by the Sky View Survey Analysis System at the Goddard Space Flight Center. One of us (WP) thanks the CONICET for the grant Nr.2496/11-12-00 to participate in the Instituto de Astrofísica de Andalucía at Granada, Spain for a month as a cooperation project CONICET of Argentina/CSIC of Spain. He also thanks ESO for partial support at the miniworkshop "The GB and other large star forming complexes" at Garching in October 24-26, 2001. He also thanks his wife Delia for her patience to allow this dedication to the paper although being formally retired for several years.

\section{References}

Arnal, E. M., Bajaja, E., Larrarte, J. J., Morras, R., \& Pöppel, W. G. L. 2000, A\&AS, 142, 35

Bajaja, E., Pöppel, W. G. L., Morras, R., \& Arnal, E. M. 2000, ASP Conf. Ser., 218,401

Berkhuijsen, E. M., Haslam, C. G. T., \& Salter, C. J. 1971, A\&A, 14, 252 Blaauw, A. 1964, ARA\&A, 2, 213

Blaauw, A. 1991, in The Physics of Star Formation and Early Stellar Evolution, ed. N. Kylafis, \& Ch. J. Lada, NATO ASI Ser. C, 342, 125

Bobylev, V. V. 2006, Astron. Lett., 32, 816

Breitschwerdt, D., Freyberg, M. J., \& Egger, R. 2000, A\&A, 361, 303 Cappa de Nicolau, C. E., \& Pöppel, W. G. L. 1986, A\&A, 164, 274 (CN\&P) Cleary, M. N., Heiles, C., \& Haslam, C. G. T. 1979, A\&AS, 36, 95

Combi, J. A., Testori, J. C., Romero, G. E., \& Colomb, F. R. 1995, A\&A, 296, 514

Corradi, W. J. B., Franco, G. A. P., \& Knude, J. 2004, MNRAS, 347, 1065

Crawford, I. A. 2000, MNRAS, 317, 996

Dame, T. M., \& Thaddeus, P. 1985, ApJ, 297, 751

Dame, T. M., Ungerechts, H. G., Cohen, R. S., et al. 1987, ApJ, 322, 706

Dame, T. M., Hartmann, D., \& Thaddeus, P. 2001, ApJ, 547, 792

de Bruijne, J. H. J. 1999, MNRAS, 310, 585

de Geus, E. J. 1992, A\&A, 262, 258 
W. G. L. Pöppel et al.: The interstellar medium surrounding the Scorpius-Centaurus association revisited

de Geus, E. J., \& Burton, W. B. 1991, A\&A, 246, 559

de Geus, E. J., Bronfman, L., \& Thaddeus, P. 1990, A\&A, 231, 137

de Zeeuw, P. T., Hoogerwerf, R., de Bruijne, J. H. J., et al. 1999, AJ, 117, 354

Egger, R. J. 1998, IAU Coll., 166, 287

Egger, R. J., \& Aschenbach, B. 1995, A\&A, 294, L25

Fejes, I. 1971, A\&A, 11, 163

Fernández, D. F. 2005, Doctoral Thesis, Universitat de Barcelona

Fernández, D. F., Figueras, F., \& Torra, J. 2008, A\&A, 480, 735

Franco, G. A. P. 1989, A\&A, 223, 313

Frisch, P. C. 1998, IAU Coll., 166, 269

Hartmann, D., \& Burton, W. B. 1997, Atlas of Galactic Neutral Hydrogen (Cambridge Univ. Press)

Hartmann, D., Magnani, L., \& Thaddeus, P. 1998, ApJ, 492, 205

Heiles, C. 1998, ApJ, 498, 689

Heiles, C., \& Jenkins, E. B. 1976, A\&A, 46, 333

Hoogerwerf, R., de Bruijne, J. H. J., \& de Zeeuw, P. T. 2000, ApJ, 544, L133

Kalberla, P. M. W., Arnal, E. M., Bajaja, E., et al. 2004, ed. D. Clemes, \& T. Brainerd, ASP Conf. Ser., in press

Kawamura, A., Onishi, T., Mizuno, A., Ogawa, H., \& Fukui, Y. 1999, PASJ, 51, 851

Lallement, R., Welsh, B. Y., Vergely, J. L., Crifo, F., \& Sfeir, D. 2003, A\&A, 411,447

Lindblad, P. O. 1967, BAN, 19, 34

Lindblad, P. O. 2000, A\&A, 363, 154

Lindblad, P. O., Grape, K., Sandqvist, A., \& Schober, J. 1973, A\&A, 24, 309

Magnani, L., Hartmann, D., \& Speck, B. G. 1996, ApJS, 106, 447

Magnani, L., Hartmann, D., Holcomb, S. L., Smith, L. E., \& Thaddeus, P. 2000, ApJ, 535, 167

Maíz-Apellániz, J. 2001, ApJ, 560, L83

Mamajek, E. E., Lawson, W. A., \& Feigelson, E. D. 2000, ApJ, 544, 356

Mamajek, E. E., Meyer, M. R., \& Liebert, J. 2002, AJ, 124, 1670
Mizuno, A., Yamaguchi, R., Tachihara, K., et al. 2001, PASJ, 53, 1071

Morras, R. 1980, A\&A, 92, 315

Morras, R. 1981, AJ, 86, 875

Olano, C. A. 1982, A\&A, 112, 195

Ortega, V. G., Jilinski, E., de la Reza, R., et al. 2009, AJ, 137, 3922

Perrot, C. A., \& Grenier, I. A. 2003, A\&A, 404, 519 (P\&G)

Pöppel, W. G. L. 1997, Fund. Cosmic Phys., 18, 1

Pöppel, W. G. L., \& Marronetti, P. 2000, A\&A, 358, 299 (P\&M)

Pöppel, W. G. L., Marronetti, P., \& Benaglia, P. 1994, A\&A, 287, 601

Preibisch, T., Guenther, E., Zinnecker, H., et al. 1998, A\&A, 333, 619

Preibisch, T., Brown, A. G. A., Bridges, T., Guenther, E., \& Zinnecker, H. 2002, AJ, 124, 404

Redfield, S., \& Linsky, J. L. 2002, ApJS, 139, 439

Reis, W., \& Corradi, W. J. B. 2008, A\&A, 486, 471

Reynoso, E. M., \& Dubner, G. M. 1997, A\&AS, 123, 31

Rizzo, J. R., Morras, R., \& Arnal, E. M. 1998, MNRAS, 300, 497

Sancisi, R., \& van Woerden, H. 1970, A\&A, 5, 135 (SvW)

Sandqvist, A., Lindblad, P. O., \& Lindroos, K. P. 1976, Third European Astron. Meeting, Tbilisi, 520

Sembach, K. R., Wakker, B. P., Savage, B. D., et al. 2003, ApJS, 146, 165

Slesnick, C. L., Hillenbrand, L. A., \& Carpenter, J. M. 2008, ApJ, 688, 377

Smith, R. K., \& Cox, D. P. 2001, ApJS, 134, 283

Snowden, S. L., Egger, R., Freyberg, M. J., et al. 1997, ApJ, 485, 125

Sodroski, T. J., Kerr, F. J., \& Sinha, R. P. 1985, IAU Symp., 106, 345

Stothers, R., \& Frogel, J. A. 1974, AJ, 79, 456

Tachihara, K., Toyoda, S., Onishi, T., et al. 2001, PASJ, 53, 1081

Testori, J. C., Reich, P., \& Reich, W. 2008, A\&A, 484, 733

Vergely, J. L., Freire Ferrero, R., Siebert, A., \& Valette, B. 2001, A\&A, 366, 1016

Weaver, H. 1979, IAU Symp., 84, 295

Wolleben, M. 2007, ApJ, 664, 349 


\section{Appendix A: Data of optical interstellar absorption lines}

The data used for deriving 18 upper limits and 1 lower limit of the distances of IS features (see Sect. 4.2) are presented in Table A.1, panels 1-19. The results derived from panels 1-15 are presented in Fig. A.1, namely panels $1-13$ corresponding to H I features listed in Table 2 (see Cols. 6-8), and panels 14 and 15 referring to the UCL-cap and the US-cap, respectively (see Sect. 5). Panels 16 (with the only lower limit) and 17 refer to H I features of intermediate velocities of Table 2; panels 18 and 19 refer to IS features in the Galactic quadrant II, and to the Sancisi \& van Woerden cloud, respectively (see Sect. 5).

The meaning of the columns is the following:

1) panel;

2) stellar identification;

$3,4)$ galactic coordinates;

5) stellar distance (h or d, indicates a Hipparcos-value with an uncertainty smaller or larger than 20\%, respectively);

6) observed IS species;

7) IS radial velocity;

8) resolution;

9) references, which are listed at the end;

10) comments. See the text for more details. 
W. G. L. Pöppel et al.: The interstellar medium surrounding the Scorpius-Centaurus association revisited

Table A.1. Data base of optical IS absorption lines.

\begin{tabular}{|c|c|c|c|c|c|c|c|c|c|}
\hline $\begin{array}{l}\mathrm{Pa}- \\
\text { nel } \\
(1)\end{array}$ & $\begin{array}{l}\text { HD } \\
\text { (2) }\end{array}$ & $\begin{array}{l}l \\
\text { (o) } \\
\text { (3) }\end{array}$ & $\begin{array}{l}b \\
\text { (o) } \\
\text { (4) }\end{array}$ & $\begin{array}{l}d \\
(\mathrm{pc}) \\
(5)\end{array}$ & sp. & $\begin{array}{l}V \\
\left(\mathrm{~km} \mathrm{~s}^{-1}\right) \\
(7)\end{array}$ & $\begin{array}{l}\delta V \\
\left(\mathrm{~km} \mathrm{~s}^{-1}\right) \\
(8)\end{array}$ & $\begin{array}{l}\text { Ref. } \\
\text { (9) }\end{array}$ & $\begin{array}{l}\text { Comments } \\
\text { (10) }\end{array}$ \\
\hline \multirow[t]{30}{*}{1} & 116658 & 316.1 & +50.8 & $80 \mathrm{~h}$ & NaI & -7.3 & 3 & 42 & $\alpha$ Vir \\
\hline & 140873 & 365.5 & +39.0 & $125 \mathrm{~h}$ & & +3.3 & 5 & 40 & \\
\hline & \multirow[t]{3}{*}{141569} & \multirow[t]{3}{*}{364.2} & \multirow[t]{3}{*}{+36.9} & \multirow[t]{3}{*}{$99 \mathrm{~h}$} & & +0.4 & 4 & 3 & \\
\hline & & & & & & +0.1 & 0.3 & 8 & \\
\hline & & & & & KI & +0.2 & 0.3 & 8 & \\
\hline & 146931 & 366.2 & +29.1 & $279 d$ & $\mathrm{NaI}$ & $-12.7,-0.7$ & 4.0 & 3 & \\
\hline & \multirow[t]{10}{*}{149757} & \multirow[t]{10}{*}{366.3} & \multirow[t]{10}{*}{+23.6} & \multirow[t]{10}{*}{$140 \mathrm{~h}$} & & $-15.4,-0.7$ & 6.0 & 5 & $\zeta \mathrm{Oph}$ \\
\hline & & & & & & $-14.7,-14.3,-6.6,-5.1,-2.4,-1.0$ & 0.5 & 15 & \\
\hline & & & & & & $0.0,+1.3,+2.9,+5.0$ & 0.5 & 15 & \\
\hline & & & & & & $-14.0,-12.0,-5.0,-2,-0.5,+3$ & 0.7 & 16 & \\
\hline & & & & & & -2.5 & 4.5 & 7 & \\
\hline & & & & & & $-15.0,-2.4$ & 4 & 3 & \\
\hline & & & & & KI & $-1.0,0.0,+1.3$ & 1.1 & 33 & \\
\hline & & & & & & -1.2 & 8 & 34 & \\
\hline & & & & & $\mathrm{NaI}$ & $-13.5,-11,-3.5,-0.4,+1.4,+5$ & 0.5 & 47 & \\
\hline & & & & & KI & $-4.5,-2.4,-0.9,+0.2,+1.5$ & 0.8 & 36 & \\
\hline & \multirow[t]{3}{*}{116658} & \multirow[t]{3}{*}{316.1} & \multirow[t]{3}{*}{+50.8} & $80 \mathrm{~h}$ & $\mathrm{Ca}$ II & $-7.1,+0.8$ & 3 & 42 & $\alpha$ Vir \\
\hline & & & & & & $-8.5,-1.5,-0.1$ & 2 & 28 & \\
\hline & & & & & & $-7.1,+0.8$ & 3 & $52 \mathrm{c}$ & \\
\hline & 120086 & 329.6 & +57.5 & $300 d$ & & $-18.4,-4.9$ & 6 & 2 & \\
\hline & & & & & Ti II & $-14.2,-1.7$ & 6 & 2 & \\
\hline & 141569 & 364.2 & +36.9 & $99 \mathrm{~h}$ & $\mathrm{Ca}$ II & $-15.1,-0.1$ & 0.3 & 8 & \\
\hline & 148833 & 368.0 & +26.6 & $123 \mathrm{~h}$ & & $-11.4,+1.2$ & 6 & 2 & \\
\hline & 149757 & 366.3 & +23.6 & $140 \mathrm{~h}$ & & -0.5 & 6 & 5 & $\zeta \mathrm{Oph}$ \\
\hline & & & & & & -14 & 3.7 & 14 & \\
\hline & & & & & & $-17.4,-14.6,-12.1,-8.4,-4.5,-2.2$ & 0.7 & 16 & \\
\hline & & & & & & $-0.4,+1.7$ & 0.7 & 16 & \\
\hline & & & & & & $-13.7,-1.2$ & 4.5 & 7 & \\
\hline & & & & & Ti II & +1.6 & 4.5 & 7 & \\
\hline & & & & & $\mathrm{Ca}$ II & -2 & 4 & 48 & 4 components \\
\hline 2 & 142039 & 372.9 & +41.1 & 90 & Ca II & $-2.6,+17.8,+37.3$ & 4 & 3 & \\
\hline & 142930 & 373.1 & +39.7 & $132 \mathrm{~h}$ & & $-8.1,-0.7$ & 4 & 3 & \\
\hline & 153212 & 378.0 & +24.4 & $242 \mathrm{~d}$ & & $+0.4,+5.2$ & 4 & 3 & \\
\hline & 153605 & 378.7 & +24.1 & 150 & & $0.0,+4.7$ & 4 & 3 & \\
\hline & 156825 & 378.3 & +18.3 & $100 \mathrm{~h}$ & & -8.6 & 4 & 3 & \\
\hline & 157280 & 378.0 & +17.4 & 140 & & $-9.3,0.0,+6.4$ & 4 & 3 & \\
\hline & 157333 & 379.0 & +17.8 & 130 & & $-9.2,-1.0,-7.1,+3.5$ & 4 & 3 & \\
\hline & 157841 & 376.8 & +15.7 & $175 \mathrm{~h}$ & & $-10.2,-1.0 .+5.0,-0.5$ & 4 & 3 & \\
\hline & & & & & $\mathrm{NaI}$ & $-8.9,+1.9,+4.7$ & 0.3 & 8 & \\
\hline & & & & & $\mathrm{Ca}$ II & $-15.0,-8.7,-2.2,+4.7,+1.9$ & 0.3 & 8 & \\
\hline & & & & & K I & $-2.0,+1.9$ & 0.3 & 8 & \\
\hline 3 & 93474 & 270.0 & +30.3 & $163 \mathrm{~h}$ & $\mathrm{NaI}$ & -5.0 & 4.0 & 3 & \\
\hline & 94473 & 272.8 & +29.2 & $278 \mathrm{~d}$ & & $-20.4,-3.4,+1.4$ & 4.0 & 3 & \\
\hline & 116658 & 316.1 & +50.8 & $80 \mathrm{~h}$ & & -7.3 & 3.0 & 42 & \\
\hline & 97277 & 274.8 & +34.5 & $82 \mathrm{~h}$ & $\mathrm{Ca}$ II & $-6.1,+1.4$ & 3 & $52 \mathrm{c}$ & \\
\hline & 104337 & 286.9 & +41.6 & 460 & Ti II & $-2,-9$ & 3.7 & 14 & 1 \\
\hline & 106625 & 291.0 & +44.5 & $51 \mathrm{~h}$ & $\mathrm{Ca}$ II & -4.1 & 0.7 & 16 & \\
\hline & 116658 & 316.1 & +50.8 & $80 \mathrm{~h}$ & & $-7.1,+0.8$ & 3 & 42 & $\alpha \mathrm{Vir}$ \\
\hline & & & & & & $-8.5,-1.5,-0.1$ & 2 & 28 & \\
\hline & & & & & & $-7.1,+0.8$ & 3 & $52 \mathrm{c}$ & \\
\hline 4 & 119786 & 321.6 & +45.1 & $104 \mathrm{~h}$ & $\mathrm{NaI}$ & -8 & 5.5 & 1 & \\
\hline & 142983 & 356.4 & +28.6 & $157 \mathrm{~h}$ & & $-12.6,-4.0,+2.1$ & 3.6 & 4 & \\
\hline & 144708 & 359.4 & +27.9 & $131 \mathrm{~h}$ & & -0.9 & 5.0 & 40 & \\
\hline & 148184 & 358 & +21 & $150 \mathrm{~h}$ & & $-14.2,-7.7,+1.8$ & 3.6 & 4 & \\
\hline & & & & & & +0.6 & 6 & 5 & \\
\hline & & & & & & $-9.8,-8.8,-8.0,-6.8,-4.3,-1.3$ & 0.5 & 15 & \\
\hline & & & & & & $+0.4,+2.0,+3.2,+4.8$ & 0.5 & 15 & \\
\hline & & & & & K I & $-1.3,0.0,+0.9,+2.8,+4.0$ & 1.1 & 33 & \\
\hline & & & & & $\mathrm{NaI}$ & $-12.2,-7.7,+1.6,+6.8$ & 0.5 & 47 & \\
\hline & 117880 & 316.7 & +43.2 & $208 \mathrm{~d}$ & $\mathrm{Ca}$ II & $-5.7,+2.2$ & 6 & 2 & \\
\hline & 119786 & 321.6 & +45.1 & $104 \mathrm{~h}$ & Ti II & +1 & 5.5 & 1 & \\
\hline & 142983 & 356.4 & +28.6 & $157 \mathrm{~h}$ & $\mathrm{Ca}$ II & $-13.1,-4.1,+1.9$ & 3.6 & 4 & \\
\hline & 148184 & 358 & +21 & $150 \mathrm{~h}$ & & $-15.2,-7.7,+0.8,+6.3$ & 3.6 & 4 & \\
\hline & & & & & & +0.8 & 6 & 5 & \\
\hline
\end{tabular}


Table A.1. continued.

\begin{tabular}{|c|c|c|c|c|c|c|c|c|c|}
\hline $\begin{array}{l}\mathrm{Pa}- \\
\text { nel } \\
(1)\end{array}$ & HD & $\begin{array}{l}l \\
\text { (o) } \\
(3)\end{array}$ & $\begin{array}{l}b \\
\text { (o) } \\
\text { (4) }\end{array}$ & $\begin{array}{l}d \\
\text { (pc) } \\
(5)\end{array}$ & $\begin{array}{l}\text { sp. } \\
\text { (6) }\end{array}$ & $\begin{array}{l}V \\
\left(\mathrm{~km} \mathrm{~s}^{-1}\right) \\
(7)\end{array}$ & $\begin{array}{l}\delta V \\
\left(\mathrm{~km} \mathrm{~s}^{-1}\right) \\
(8)\end{array}$ & $\begin{array}{l}\text { Ref. } \\
\text { (9) }\end{array}$ & $\begin{array}{l}\text { Comments } \\
\text { (10) }\end{array}$ \\
\hline \multirow[t]{65}{*}{5} & 119921 & 315.3 & +25.3 & $131 \mathrm{~h}$ & $\mathrm{NaI}$ & -8.6 & 3.0 & 42 & \\
\hline & 126769 & 327.0 & +28.8 & $127 \mathrm{~h}$ & & +20.0 & 4.6 & 17 & \\
\hline & 129685 & 327.7 & +22.1 & $63 \mathrm{~h}$ & & -12.8 & 3.0 & 42 & \\
\hline & 139094 & 343.0 & +23.2 & 289 & & -3.1 & 4 & 6 & \\
\hline & 141180 & 344.4 & +20.7 & 358 & & $-9.3,+1.7,+17.8$ & 4 & 6 & \\
\hline & 141637 & 346.1 & +21.7 & $160 \mathrm{~h}$ & & $-6.7,+1.0$ & 3.6 & 4 & \\
\hline & & & & & & $-8.2,-5.2,-3.5,+0.1,+2.0$ & 0.5 & 15 & \\
\hline & & & & & & $-8,-5,0,+2$ & 0.8 & 16 & \\
\hline & & & & & & $-7.3,-4.3,+1.7,+2.7$ & 0.5 & 47 & \\
\hline & & & & & & $-7.9,+0.4,+2.4$ & 1.1 & 33 & \\
\hline & 142114 & 346.9 & +21.6 & 133h & & $-8.1,-3.1$ & 4 & 4 & \\
\hline & & & & & & -9.0 & 6 & 5 & \\
\hline & 143018 & 347.2 & +20.2 & $141 \mathrm{~h}$ & & $-13.3,-8.0,-7.0,-6.0,-5.3,-3.7$ & 0.5 & 15 & \\
\hline & & & & & & the & 0.5 & 15 & \\
\hline & & & & & & $-14,-8,-6,-3,+2$ & 0.8 & 16 & \\
\hline & & & & & K I & +8.9 & 8 & 34 & \\
\hline & & & & & $\mathrm{NaI}$ & $-12.6,-6.1,-4.1$ & 0.5 & 47 & \\
\hline & 143275 & 350.1 & +22.5 & $123 \mathrm{~h}$ & K I & $-4.9,-1.0$ & 1.1 & 33 & \\
\hline & & & & & $\mathrm{NaI}$ & $-11.6,+4.9$ & 0.5 & 47 & \\
\hline & & & & & & -2.1 & 5 & 47 & \\
\hline & 144217 & 353.2 & +23.6 & $163 \mathrm{~h}$ & & $-12.6,-11.2,-2.0,+0.6,+2.5,+4.0$ & 0.5 & 15 & \\
\hline & & & & & & $-13,-11,+1,+2.5$ & 0.8 & 16 & \\
\hline & & & & & K I & +1.9 & 8 & 34 & \\
\hline & & & & & & $-0.2,+1.8$ & 1.1 & 33 & \\
\hline & & & & & & $-0.9,+0.9$ & 1.8 & 35 & \\
\hline & & & & & $\mathrm{NaI}$ & $-12.6,-0.4$ & 5 & 40 & \\
\hline & & & & & & $-11.2,+0.8,+2.8$ & 0.5 & 47 & \\
\hline & 144470 & 352.8 & +22.8 & $130 \mathrm{~h}$ & & $-13.4,-5.4,+1.6$ & 4 & 4 & \\
\hline & & & & & & -0.4 & 6 & 5 & \\
\hline & & & & & K I & $-1.9,-0.5,+1.0,+2.2,+4.7$ & 1 & 33 & \\
\hline & & & & & $\mathrm{NaI}$ & $-12.9,-5.9,-1.4$ & 0.5 & 47 & \\
\hline & & & & & & +2.6 & 4.0 & 47 & \\
\hline & 144844 & 350.7 & +20.4 & $131 \mathrm{~h}$ & & $-12.6,-0.2$ & 5 & 40 & \\
\hline & 145102 & 348.6 & +17.9 & $175 \mathrm{~h}$ & & $-12.0,+3.8$ & 4.0 & 6 & \\
\hline & 145482 & 348.1 & +16.8 & $143 \mathrm{~h}$ & & $-10.3,+7.5$ & 5 & 40 & \\
\hline & 145502 & 354.6 & +22.7 & $134 \mathrm{~h}$ & K I & $-1.5,+0.9,+2.6,+4.2$ & 1.1 & 33 & \\
\hline & & & & & & +0.1 & 8 & 34 & \\
\hline & & & & & & $-2.2,0.0,+1.8$ & 1.8 & 35 & \\
\hline & & & & & $\mathrm{NaI}$ & $-13.4,-5.4$ & 0.5 & 47 & \\
\hline & & & & & & +1.6 & 8 & 47 & \\
\hline & & & & & & +8.6 & 0.5 & 47 & \\
\hline & & & & & & $-14.7,+0.5$ & 5 & 40 & \\
\hline & 146284 & 351.6 & +18.7 & $264 \mathrm{~h}$ & & $-14.2,+1.6$ & 4.0 & 6 & \\
\hline & 147084 & 352.3 & +18.1 & 170 & K I & $-1.7,+2.3,+2.9,+3.9$ & 1.1 & 33 & \\
\hline & 147165 & 351.3 & +17.0 & $225 \mathrm{~h}$ & $\mathrm{NaI}$ & -3.7 & 0.5 & 47 & \\
\hline & & & & & & +4.3 & 6 & 47 & \\
\hline & & & & & K I & +2.7 & 8 & 34 & \\
\hline & & & & & & $+3.4,+5.1,+6.6$ & 1.1 & 33 & \\
\hline & 147889 & 352.9 & +17.0 & $136 \mathrm{~h}$ & & +2.6 & 8 & 34 & \\
\hline & $147933 / 4$ & 353.7 & +17.7 & 149 & & +1.9 & 8 & 34 & ref.54 \\
\hline & & & & & & +2.7 & 1.8 & 35 & \\
\hline & & & & & & $-0.7,+0.1,+1.5,+2.5,+3.7,+4.3$ & 1.1 & 33 & \\
\hline & 148605 & 353.1 & +15.8 & $120 \mathrm{~h}$ & K I & +2.6 & 1.1 & 33 & \\
\hline & & & & & & +10.1 & 8 & 34 & \\
\hline & 149438 & 351.5 & +12.8 & $132 \mathrm{~h}$ & $\mathrm{NaI}$ & $-8.6,+1.4,+5.2$ & 0.5 & 47 & \\
\hline & & & & & & $-0.5,+0.6,+1.5,+3.1,+4.9$ & 0.5 & 15 & \\
\hline & & & & & & $-1,+1,+5$ & 0.8 & 16 & \\
\hline & & & & & & +9.4 & 8 & 34 & \\
\hline & 129685 & 327.7 & +22.1 & $63 \mathrm{~h}$ & $\mathrm{CaII}$ & $-22.9,-13.5$ & 3.0 & 42 & \\
\hline & 141637 & 346.1 & +21.7 & $160 \mathrm{~h}$ & & +1.0 & 3.6 & 4 & \\
\hline & & & & & & $-3.6,-0.4,+0.3,+1.0,+2.3,+3.0$ & 0.8 & 16 & \\
\hline & 142114 & 346.9 & +21.6 & $133 \mathrm{~h}$ & & -8.1 & 3.6 & 4 & \\
\hline & 143275 & 350.1 & +22.5 & $123 \mathrm{~h}$ & & $-5,-2$ & 1.0 & 48 & \\
\hline & 144217 & 353.2 & +23.6 & $163 \mathrm{~h}$ & & $-21.5,-18.1,-13.2,-10.1,-4.5$, & 0.8 & 16 & \\
\hline & & & & & & $-0.1,+0.4,+1.7,+2.4$ & 0.8 & 16 & \\
\hline
\end{tabular}


W. G. L. Pöppel et al.: The interstellar medium surrounding the Scorpius-Centaurus association revisited

Table A.1. continued.

\begin{tabular}{|c|c|c|c|c|c|c|c|c|c|}
\hline $\begin{array}{l}\mathrm{Pa}- \\
\text { nel } \\
\text { (1) }\end{array}$ & $\begin{array}{l}\text { HD } \\
\text { (2) }\end{array}$ & $\begin{array}{l}l \\
\text { (o) } \\
\text { (3) }\end{array}$ & $\begin{array}{l}b \\
\text { (o) } \\
(4)\end{array}$ & $\begin{array}{l}d \\
(\mathrm{pc}) \\
(5)\end{array}$ & $\begin{array}{l}\text { sp. } \\
\text { (6) }\end{array}$ & $\begin{array}{l}V \\
\left(\mathrm{~km} \mathrm{~s}^{-1}\right) \\
(7)\end{array}$ & $\begin{array}{l}\delta V \\
\left(\mathrm{~km} \mathrm{~s}^{-1}\right) \\
(8)\end{array}$ & $\begin{array}{l}\text { Ref. } \\
\text { (9) }\end{array}$ & $\begin{array}{l}\text { Comments } \\
\text { (10) }\end{array}$ \\
\hline \multirow{13}{*}{ (1) } & & & & & & $-10,+2$ & 1.0 & 48 & \\
\hline & & & & & Ti II & +3 & 3.7 & 14 & \\
\hline & 144470 & 352.8 & +22.8 & $130 \mathrm{~h}$ & CaII & $-13.4,+1.1$ & 3.6 & 4 & \\
\hline & & & & & & +1.1 & 6.0 & 5 & \\
\hline & & & & & & +3 & 1.0 & 48 & \\
\hline & & & & & Ti II & $-21,+3,+11$ & 3.7 & 14 & \\
\hline & 145502 & 354.6 & +22.7 & $134 \mathrm{~h}$ & & +3 & 3.7 & 14 & \\
\hline & 147165 & 351.3 & +17.0 & $225 \mathrm{~h}$ & CaII & $-20.9,-17.9,-11.6,-6.9,-2.3$, & 0.8 & 16 & \\
\hline & & & & & & $+1.5,+3.5,+4.9,+6.1$ & 0.8 & 16 & \\
\hline & & & & & & +4 & 1.0 & 48 & \\
\hline & & & & & Ti II & $-5,+5$ & 3.7 & 14 & \\
\hline & 149438 & 351.5 & +12.8 & $132 \mathrm{~h}$ & & $-16,-1,+4$ & 1.0 & 48 & \\
\hline & & & & & & +0.6 & 0.7 & 16 & \\
\hline \multirow[t]{7}{*}{6} & 49835 & 289.9 & -27.1 & $285 \mathrm{~d}$ & $\mathrm{NaI}$ & $-1.0,+5.0$ & 4 & 3 & \\
\hline & 52365 & 289.1 & -26.5 & $143 \mathrm{~h}$ & & +5.1 & 4 & 3 & \\
\hline & 57336 & 291.2 & -25.8 & $385 \mathrm{~d}$ & & $-7.2,+3.0$ & 4 & 3 & \\
\hline & 64033 & 285.9 & -22.4 & $158 \mathrm{~h}$ & & +6 & 4 & 3 & 1 \\
\hline & 64409 & 286.2 & -22.3 & $279 d$ & & -11.4 & 4 & 3 & \\
\hline & 65575 & 266.7 & -12.3 & $119 \mathrm{~h}$ & & -0.3 & 3.1 & 38 & \\
\hline & 66920 & 285.7 & -21.2 & $125 \mathrm{~h}$ & & +4.5 & 4 & 3 & \\
\hline \multirow[t]{15}{*}{7} & 105435 & 296.0 & +11.6 & $121 \mathrm{~h}$ & & $-6.5,0.0$ & 3.6 & 4 & clump \\
\hline & 108483 & 299.1 & +12.5 & $136 \mathrm{~h}$ & & $-8.0,-2.5$ & 3.6 & 4 & \\
\hline & 120324 & 314.2 & +19.1 & $162 \mathrm{~h}$ & & -10.6 & 3.6 & 4 & branch \\
\hline & 121263 & 314.1 & +14.2 & $118 \mathrm{~h}$ & & -13.8 & 3 & 42 & \\
\hline & 121743 & 316.0 & +19.1 & $143 \mathrm{~h}$ & & -10.1 & 4.6 & 17 & \\
\hline & & & & & & $-14.3,-9.0,-2.5$ & 3 & 42 & \\
\hline & 125238 & 318.5 & +14.1 & $108 \mathrm{~h}$ & & -14.3 & 4.6 & 17 & \\
\hline & 105435 & 296.0 & +11.6 & $121 \mathrm{~h}$ & $\mathrm{Ca}$ II & $-6.5,0.0$ & 3.6 & 4 & clump \\
\hline & & & & & & $-8.1,+23.1$ & 4.5 & 7 & \\
\hline & 105937 & 296.8 & +10.0 & $105 \mathrm{~h}$ & & $-20.5,-15.7,-9.5,-7.3,-4.2$ & 0.4 & 43 & \\
\hline & 108483 & 299.1 & +12.5 & $136 \mathrm{~h}$ & & $-8.0,-2.5$ & 3.6 & 4 & \\
\hline & 115892 & 309.4 & +25.8 & $18 \mathrm{~h}$ & & -19.1 & 5 & 42 & branch \\
\hline & & & & & & -19.6 & 0.4 & 43 & \\
\hline & 121263 & 314.1 & +14.2 & $118 \mathrm{~h}$ & & $-14.4,-4.6$ & 5 & 42 & \\
\hline & 121743 & 316.0 & +19.1 & $143 \mathrm{~h}$ & & $-16.1,-8.7,-2.2$ & 5 & 42 & \\
\hline \multirow[t]{9}{*}{8} & 184597 & 366.8 & -23.1 & $265 d$ & $\mathrm{NaI}$ & $+1.4,+16.7$ & 5 & 46 & \\
\hline & 185163 & 369.4 & -22.8 & 288 & & $+1.3,+13.4$ & 5 & 46 & \\
\hline & 186417 & 369.3 & -24.4 & $234 \mathrm{~h}$ & & +4.0 & 5 & 46 & \\
\hline & 186500 & 368.3 & -24.8 & $167 \mathrm{~h}$ & & $+1.5,+7.2$ & 5 & 46 & \\
\hline & 187741 & 368.7 & -26.2 & 450 & & +5.6 & 5 & 46 & \\
\hline & 188220 & 385.3 & -21.0 & 220 & & +2.8 & 4 & 3 & \\
\hline & 189103 & 365.5 & -28.5 & $189 \mathrm{~h}$ & & +5.5 & 4.7 & 17 & \\
\hline & 189103 & 365.5 & -28.5 & $189 \mathrm{~h}$ & $\mathrm{Ca} I I$ & $-14.0,+5.5$ & 4.5 & 7 & \\
\hline & & & & & Ti II & +2.0 & 4. & 7 & \\
\hline \multirow[t]{16}{*}{9} & 22252 & 282.2 & -43.7 & $261 \mathrm{~h}$ & $\mathrm{NaI}$ & $-0.6,+0.2,+8.8,+19.2$ & 3.5 & 11 & \\
\hline & & & & & & $-5,+1.5,+8$ & 0.3 & 12 & 1 \\
\hline & 22488 & 282.3 & -43.4 & $207 \mathrm{~h}$ & & +8.8 & 3.5 & 11 & \\
\hline & 23509 & 281.4 & -42.8 & $214 \mathrm{~h}$ & & $-0.6,+0.2,+8.8$ & 3.5 & 11 & \\
\hline & 25938 & 285.2 & -38.8 & $101 \mathrm{~h}$ & & +9.9 & 3.5 & 11 & \\
\hline & 26109 & 284.1 & -39.1 & $234 \mathrm{~h}$ & & +4.6 & 3.5 & 11 & \\
\hline & 26594 & 287.2 & -37.3 & $229 \mathrm{~h}$ & & $+1.1,+6.7$ & 3.5 & 11 & \\
\hline & 29769 & 285.3 & -35.7 & $168 \mathrm{~h}$ & & +5.4 & 3.5 & 11 & \\
\hline & 49835 & 289.9 & -27.1 & $285 \mathrm{~d}$ & & $-1.0,+5.0$ & 4 & 3 & \\
\hline & 52365 & 289.1 & -26.5 & $143 \mathrm{~h}$ & & +5.1 & 4 & 3 & \\
\hline & 57336 & 291.2 & -25.8 & $385 \mathrm{~d}$ & & $-7.2,+3.0$ & 4 & 3 & \\
\hline & $\mathrm{SAO}$ & 295.0 & -21.0 & $165 \mathrm{~h}$ & & +4.2 & 5 & 55 & 377063 \\
\hline & 22252 & 282.2 & -43.7 & $261 \mathrm{~h}$ & $\mathrm{Ca}$ II & $-8.0,-3.7,+0.7,+4.9,+8.6,+18.6$ & 3.5 & 11 & \\
\hline & & & & & & $-7.2,-3.8,-3.7,+0.5,+0.9,+4.9$ & 0.3 & 12 & \\
\hline & & & & & & $+6.7,+8.5,+8.8,+18.0$ & 0.3 & 12 & \\
\hline & 26109 & 284.1 & -39.1 & $234 \mathrm{~h}$ & & +3.8 & 3.5 & 11 & \\
\hline \multirow[t]{5}{*}{10} & 138138 & 337.1 & +18.2 & $100 \mathrm{~h}$ & $\mathrm{NaI}$ & -12.0 & 4 & 6 & \\
\hline & 141905 & 336.7 & +10.8 & $204 \mathrm{~h}$ & & +5.6 & 4 & 6 & \\
\hline & 142983 & 356.4 & +28.6 & $157 \mathrm{~h}$ & & $-12.6,-4.0,+2.1$ & 3.6 & 4 & \\
\hline & 143927 & 339.9 & +10.8 & $146 \mathrm{~h}$ & & +4.8 & 4 & 6 & \\
\hline & 144217 & 353.2 & +23.6 & $163 \mathrm{~h}$ & & $-12.6,-11.2,-2.0,+0.6,+2.5,+4.0$ & 0.5 & 15 & \\
\hline
\end{tabular}


Table A.1. continued.

\begin{tabular}{|c|c|c|c|c|c|c|c|c|c|}
\hline \multirow[t]{50}{*}{$\begin{array}{l}\mathrm{Pa}- \\
\text { nel } \\
\text { (1) }\end{array}$} & $\begin{array}{l}\text { HD } \\
\text { (2) }\end{array}$ & $\begin{array}{l}l \\
\text { (o) } \\
\text { (3) }\end{array}$ & $\begin{array}{l}b \\
\text { (o) } \\
\text { (4) }\end{array}$ & $\begin{array}{l}d \\
(\mathrm{pc}) \\
(5)\end{array}$ & $\begin{array}{l}\text { sp. } \\
\text { (6) }\end{array}$ & $\begin{array}{l}V \\
\left(\mathrm{~km} \mathrm{~s}^{-1}\right) \\
(7)\end{array}$ & $\begin{array}{l}\delta V \\
\left(\mathrm{~km} \mathrm{~s}^{-1}\right) \\
(8)\end{array}$ & $\begin{array}{l}\text { Ref. } \\
\text { (9) }\end{array}$ & $\begin{array}{l}\text { Comments } \\
\text { (10) }\end{array}$ \\
\hline & & & & & & $-13,-11,+1,+2.5$ & 0.8 & 16 & \\
\hline & & & & & K I & +1.9 & 8 & 34 & \\
\hline & & & & & & $-0.2,+1.8$ & 1.1 & 33 & \\
\hline & & & & & & $-0.9,+0.9$ & 1.8 & 35 & \\
\hline & & & & & $\mathrm{NaI}$ & $-12.6,-0.4$ & 5 & 40 & \\
\hline & & & & & & $-11.2,+0.8,+2.8$ & 0.5 & 47 & \\
\hline & 144708 & 359.4 & +27.9 & $131 \mathrm{~h}$ & & -0.9 & 5 & 40 & \\
\hline & 145502 & 354.6 & +22.7 & $134 \mathrm{~h}$ & & +0.1 & 8 & 34 & \\
\hline & & & & & K I & $+1.8,0.0,-2.2$ & 1.8 & 35 & \\
\hline & & & & & $\mathrm{NaI}$ & $-14.7,+0.5$ & 5 & 40 & \\
\hline & & & & & & $-13.4,-5.4$ & 0.5 & 47 & \\
\hline & & & & & & +1.6 & 8 & 47 & \\
\hline & & & & & & +8.6 & 0.5 & 47 & \\
\hline & & & & & & $-1.5,+0.9,+2.6,+4.2$ & 1.1 & 33 & \\
\hline & 147009 & 355.5 & +20.9 & $161 \mathrm{~h}$ & & +3.9 & 8 & 34 & \\
\hline & 147165 & 351.3 & +17.0 & $225 \mathrm{~h}$ & & -3.7 & 0.5 & 47 & \\
\hline & & & & & & +4.3 & 6 & 47 & \\
\hline & & & & & K I & $+3.4,+5.1,+6.6$ & 1.1 & 33 & \\
\hline & & & & & & +2.7 & 8 & 34 & \\
\hline & 147889 & 352.9 & +17.0 & $136 \mathrm{~h}$ & & +2.6 & 8 & 34 & \\
\hline & 147933 & 353.7 & +17.7 & 149 & & +1.9 & 8 & 34 & \\
\hline & & & & & & +2.7 & 1.8 & 35 & \\
\hline & & & & & & $-0.7,+0.1,+1.5,+2.5,+3.7,+4.3$ & 1.1 & 33 & \\
\hline & 148184 & 357.9 & +20.7 & $150 \mathrm{~h}$ & $\mathrm{NaI}$ & +0.6 & 6 & 5 & \\
\hline & & & & & & $-9.8,-8.8,-8.0,-6.8,-4.3$ & 0.5 & 15 & \\
\hline & & & & & & $-1.3,+0.4,+2.0,+3.2,+4.8$ & 0.5 & 15 & \\
\hline & & & & & K I & $-1.3,0.0,+0.9,+2.8,+4.0$ & 1.1 & 33 & \\
\hline & & & & & & -0.4 & 8 & 34 & \\
\hline & & & & & $\mathrm{NaI}$ & $-12.2,-7.7,+6.8$ & 0.5 & 47 & \\
\hline & & & & & & +1.6 & 7 & 47 & \\
\hline & 148605 & 353.1 & +15.8 & $120 \mathrm{~h}$ & K I & +2.6 & 1.1 & 33 & \\
\hline & & & & & & +10.1 & 8 & 34 & \\
\hline & 148703 & 345.9 & +09.2 & $229 \mathrm{~h}$ & $\mathrm{NaI}$ & $-9.6,-1.1,+3.4,+12.4$ & 3.6 & 4 & \\
\hline & 149438 & 351.5 & +12.8 & $132 \mathrm{~h}$ & & $-0.5,+0.6,+1.5,+3.1,+4.9$ & 0.5 & 15 & \\
\hline & & & & & & $-1,+1,+5$ & 0.8 & 16 & \\
\hline & & & & & K I & +9.4 & 8 & 34 & \\
\hline & 142983 & 356.4 & +28.6 & $157 \mathrm{~h}$ & Ca II & $-13.1,-4.1,+1.9$ & 3.6 & 4 & \\
\hline & 144217 & 353.2 & +23.6 & $163 \mathrm{~h}$ & & $-10,+2$ & 1.0 & 48 & \\
\hline & & & & & Ti II & +3 & 3.7 & 14 & \\
\hline & & & & & Ca II & $-21.5,-18.1,-13.2,-10.1,-4.5$ & 0.7 & 16 & \\
\hline & & & & & & $-0.1,+0.4,+1.7,+2.4$ & 0.7 & 16 & \\
\hline & 147165 & 351.3 & +17.0 & $225 \mathrm{~h}$ & Ti II & $-5,+5$ & 3.7 & 14 & \\
\hline & & & & & Ca II & +4 & 1.0 & 48 & \\
\hline & & & & & & $-20.9,-17.9,-11.6,-6.9,-2.3$ & 0.8 & 16 & \\
\hline & & & & & & $+1.5,+3.5,+4.9,+6.1$ & 0.8 & 16 & \\
\hline & 148184 & 357.9 & +20.7 & $150 \mathrm{~h}$ & & +0.8 & 6.0 & 5 & \\
\hline & & & & & & $-15.2,-7.7,+0.8,+6.3$ & 3.6 & 4 & \\
\hline & 148703 & 345.9 & +09.2 & $229 \mathrm{~h}$ & & $-10.6,-2.1,+2.4,+7.4$ & 3.6 & 4 & \\
\hline & 149438 & 351.5 & +12.8 & $132 \mathrm{~h}$ & & +0.6 & 0.7 & 16 & \\
\hline 11 & 80840 & 292.7 & -19.8 & 340 & $\mathrm{NaI}$ & -12.3 & 4 & 3 & \\
\hline & 92157 & 294.4 & -14.5 & $312 \mathrm{~h}$ & & $+2.7,+6.4$ & 4 & 3 & \\
\hline & 92438 & 293.7 & -13.2 & 120 & & -15.5 & 4 & 3 & \\
\hline & 92645 & 293.8 & -13.1 & 300 & & $-9.3,-2.2$ & 4 & 3 & \\
\hline & 93237 & 297.1 & -18.3 & 255 & & $+3.1,-4.9$ & 5 & 55 & \\
\hline & 94413 & 295.1 & -13.6 & 280 & & $-36.0,-27.2,-21.6,-12.9,+0.8$ & 4 & 3 & \\
\hline & 94414 & 296.3 & -15.8 & $230 \mathrm{~h}$ & $\mathrm{Ca} I I$ & $+4.8,-2.9$ & 4.2 & 10 & \\
\hline & & & & & $\mathrm{NaI}$ & +2.3 & 5 & 55 & \\
\hline & 96675 & 296.6 & -14.6 & $164 \mathrm{~h}$ & $\mathrm{Ca}$ II & +4.6 & 4.2 & 10 & \\
\hline & & & & & $\mathrm{NaI}$ & +3.8 & 5 & 55 & \\
\hline & 97048 & 297.4 & -15.9 & $175 \mathrm{~h}$ & Ca II & $-7.8,+4.5$ & 4.2 & 10 & \\
\hline & 97300 & 297.0 & -14.9 & $188 \mathrm{~h}$ & & +4.2 & 4.2 & 10 & \\
\hline & 98143 & 297.7 & -15.6 & $334 d$ & $\mathrm{NaI}$ & $+0.6,+3.8$ & 4 & 3 & \\
\hline & & & & & $\mathrm{Ca} I I$ & +4.7 & 4.2 & 10 & \\
\hline & 98294 & 296.9 & -13.5 & $331 d$ & & +1.8 & 4.2 & 10 & \\
\hline & 99759 & 297.8 & -13.9 & $243 \mathrm{~h}$ & & +1.7 & 4.2 & 10 & \\
\hline
\end{tabular}


W. G. L. Pöppel et al.: The interstellar medium surrounding the Scorpius-Centaurus association revisited

Table A.1. continued.

\begin{tabular}{|c|c|c|c|c|c|c|c|c|c|}
\hline $\begin{array}{l}\mathrm{Pa}- \\
\text { nel } \\
\text { (1) }\end{array}$ & $\begin{array}{l}\text { HD } \\
\text { (2) }\end{array}$ & $\begin{array}{l}l \\
\text { (o) } \\
\text { (3) }\end{array}$ & $\begin{array}{l}b \\
\text { (o) } \\
\text { (4) }\end{array}$ & $\begin{array}{l}d \\
(\mathrm{pc}) \\
(5)\end{array}$ & sp. & $\begin{array}{l}V \\
\left(\mathrm{~km} \mathrm{~s}^{-1}\right) \\
(7)\end{array}$ & $\begin{array}{l}\delta V \\
\left(\mathrm{~km} \mathrm{~s}^{-1}\right) \\
(8)\end{array}$ & $\begin{array}{l}\text { Ref. } \\
\text { (9) }\end{array}$ & $\begin{array}{l}\text { Comments } \\
\text { (10) }\end{array}$ \\
\hline \multirow[t]{18}{*}{12} & 157841 & 376.8 & +15.7 & $175 \mathrm{~h}$ & $\mathrm{NaI}$ & $-10.2,-1.0,+5.0$ & 4.0 & 3 & \\
\hline & 157841 & & & & & $-8.9,+1.9,+4.7$ & 0.3 & 8 & \\
\hline & 159975 & 377.0 & +12.3 & $168 \mathrm{~h}$ & & +0.6 & 0.5 & 47 & \\
\hline & 161270 & 387.5 & +16.0 & $141 \mathrm{~h}$ & K I & +1.2 & 1.8 & 35 & /89 \\
\hline & 164353 & 389.7 & +12.6 & $435 d$ & & $-3.1,-0.1,+2.0,+3.1,+5.2$ & 1.1 & 33 & \\
\hline & & & & & & $+7.2,+9.7$ & 1.1 & 33 & \\
\hline & & & & & & +2.0 & 8 & 34 & \\
\hline & & & & & $\mathrm{NaI}$ & $+3.3,+22.8$ & 0.5 & 47 & \\
\hline & 165402 & 380.3 & +06.1 & $175 \mathrm{~h}$ & & +2.5 & 5 & 40 & \\
\hline & 169033 & 379.0 & +00.7 & $188 \mathrm{~h}$ & & +1.3 & 5 & 40 & \\
\hline & 173370 & 394.0 & +02.4 & $145 \mathrm{~h}$ & & +0.2 & 4.6 & 17 & \\
\hline & & & & & & +0.1 & 5 & 40 & \\
\hline & 175869 & 395.9 & -00.1 & $338 d$ & & $-1.2,+7.4$ & 5 & 40 & \\
\hline & 157841 & 376.8 & +15.7 & $175 \mathrm{~h}$ & $\mathrm{Ca} I I$ & -0.5 & 4.0 & 3 & \\
\hline & & & & & & $-15.0,-8.7,-2.2,+1.9,+4.7$ & 0.3 & 8 & \\
\hline & 159975 & 377.0 & +12.3 & $168 \mathrm{~h}$ & & $+0.5,+4$ & 1.0 & 48 & \\
\hline & 161868 & 388.0 & +15.4 & 29 & & $-15.3,-12.2$ & 0.35 & 43 & \\
\hline & 164353 & 389.7 & +12.6 & $435 \mathrm{~d}$ & & $+2.5,+6.0,+9.5$ & 1.0 & 48 & \\
\hline \multirow[t]{14}{*}{13} & 203387 & 393.6 & -40.8 & 66 & Mg II & $-12.8,-4.1,+5.5$ & 2.6 & $52 \mathrm{a}$ & \\
\hline & 203387 & & & & Fe II & $-11.6,-4.9,+5.7$ & 2.6 & $52 \mathrm{a}$ & \\
\hline & 205637 & 391.9 & -45.0 & $203 \mathrm{~h}$ & $\mathrm{Ca}$ II & $-13.1,-4.2,+0.9$ & 6 & 2 & \\
\hline & 210191 & 397.1 & -51.8 & 350 & & $-21.6,-15.3,-6.8,+1.9$ & 6 & 2 & \\
\hline & & & & & Ti II & $-7.5,+1.8$ & 6 & 2 & \\
\hline & & & & & Ca II & $-18,0.0$ & 5.5 & 1 & \\
\hline & 205265 & 377.5 & -47.0 & $213 \mathrm{~h}$ & & -6.2 & 6 & 2 & \\
\hline & 207603 & 381.0 & -50.2 & $203 \mathrm{~h}$ & & $-13.2,-5.9,+2.3$ & 6 & 2 & \\
\hline & 209522 & 383.6 & -53.0 & 350 & & $-16.8,-7.2,+2.7$ & 6 & 2 & \\
\hline & 209522 & & & & & $-18.4,-14.8,-11.2,-5.6,+2.5$ & 0.7 & 53 & \\
\hline & 210934 & 382.6 & -55.3 & $156 \mathrm{~h}$ & & -11.6 & 6 & 2 & \\
\hline & 198174 & 379.6 & -36.3 & 279d & $\mathrm{NaI}$ & +2.8 & 4 & 3 & \\
\hline & 210191 & 397.1 & -51.8 & 350 & & $-18,0.0$ & 5.5 & 1 & \\
\hline & 209522 & 383.6 & -53.0 & 350 & & $-18.6,-12.2,-9.2,-6.3,+2.3$ & 0.7 & 53 & \\
\hline \multirow[t]{29}{*}{14} & 115892 & 309.4 & +25.8 & 18 & $\mathrm{CaII}$ & -19.1 & 5 & 42 & \\
\hline & 115892 & & & & & -19.6 & 0.4 & 43 & \\
\hline & 119921 & 315.3 & +25.3 & $131 \mathrm{~h}$ & $\mathrm{NaI}$ & -8.6 & 5 & 42 & \\
\hline & & & & & $\mathrm{Ca}$ II & $-21.3,-9.8$ & 5 & 42 & \\
\hline & 120324 & 314.2 & +19.1 & $162 \mathrm{~h}$ & $\mathrm{NaI}$ & -10.6 & 3.6 & 4 & \\
\hline & 121263 & 314.1 & +14.2 & $118 \mathrm{~h}$ & $\mathrm{Ca}$ II & $-14.4,-4.6$ & 5 & 42 & \\
\hline & & & & & $\mathrm{NaI}$ & -13.8 & 3 & 42 & \\
\hline & 121743 & 316.0 & +19.1 & $143 \mathrm{~h}$ & $\mathrm{Ca}$ II & $-16.1,-8.7,-2.2$ & 5 & 42 & \\
\hline & & & & & $\mathrm{NaI}$ & $-14.3,-9.0,-2.5$ & 3 & 42 & \\
\hline & & & & & & $-15.1,-10.1$ & 3.6 & 4 & \\
\hline & & & & & & -10.1 & 4.6 & 17 & \\
\hline & 122451 & 311.8 & +01.3 & $161 \mathrm{~h}$ & $\mathrm{Ca}$ II & $-23.0,-18.8, .13 .6,-12.1,-8.4$ & 0.4 & 43 & \\
\hline & 125238 & 318.5 & +14.1 & $108 \mathrm{~h}$ & $\mathrm{NaI}$ & -14.3 & 4.6 & 17 & \\
\hline & 125473 & 321.7 & +21.7 & $76 \mathrm{~h}$ & & $-29.8,-20.0,-9.2$ & 3 & 42 & \\
\hline & 125823 & 321.6 & +20.0 & $128 \mathrm{~h}$ & & $-11.4,-2.2,+7.8$ & 3.6 & 4 & \\
\hline & & & & & $\mathrm{Ca} I I$ & $-11.4,-3.2$ & 3.6 & 4 & \\
\hline & 127972 & 322.8 & +16.7 & $95 \mathrm{~h}$ & $\mathrm{NaI}$ & $-21.8,-11.2$ & 3 & 42 & \\
\hline & 129685 & 327.7 & +22.1 & $63 \mathrm{~h}$ & Ca II & $-22.9,-13.5$ & 5 & 42 & \\
\hline & & & & & $\mathrm{NaI}$ & -12.8 & 3 & 42 & \\
\hline & 132200 & 326.9 & +14.8 & $165 \mathrm{~h}$ & & -6.8 & 3.6 & 4 & \\
\hline & & & & & $\mathrm{Ca}$ II & -5.3 & 3.6 & 4 & \\
\hline & 133955 & 326.8 & +11.1 & $125 \mathrm{~h}$ & $\mathrm{NaI}$ & -11.3 & 3.6 & 4 & \\
\hline & 134685 & 333.0 & +19.5 & $174 \mathrm{~h}$ & & $-10.2,+5.9$ & 4 & 6 & \\
\hline & 136961 & 334.7 & +17.3 & $148 \mathrm{~h}$ & & -14.3 & 4 & 6 & \\
\hline & 137957 & 330.0 & +08.9 & $198 \mathrm{~h}$ & & $+4.5,+15.4$ & 4 & 6 & \\
\hline & 138138 & 337.1 & +18.2 & $100 \mathrm{~h}$ & & -12.0 & 4 & 6 & \\
\hline & 141905 & 336.7 & +10.8 & $204 \mathrm{~h}$ & & +5.6 & 4 & 6 & \\
\hline & 143927 & 339.9 & +10.8 & $146 \mathrm{~h}$ & & +4.8 & 4 & 6 & \\
\hline & 145037 & 335.5 & +04.5 & $106 \mathrm{~h}$ & K I & $-4.9,+5.1,+8.6$ & 8 & 34 & \\
\hline \multirow[t]{4}{*}{15} & 135230 & 344.7 & +33.3 & 200 & CaII & +30 & 6 & 2 & \\
\hline & 138485 & 349.3 & +31.1 & $236 \mathrm{~h}$ & & $-2.1,+22.4,+43.1$ & 6 & 2 & \\
\hline & & & & & Ti II & -1.1 & 6 & 2 & \\
\hline & 139094 & 343.0 & +23.2 & 289 & $\mathrm{NaI}$ & -3.1 & 4 & 6 & \\
\hline
\end{tabular}


Table A.1. continued.

\begin{tabular}{|c|c|c|c|c|c|c|c|c|c|}
\hline $\begin{array}{l}\mathrm{Pa}- \\
\text { nel } \\
(1)\end{array}$ & HD & $\begin{array}{l}l \\
\text { (o) } \\
(3)\end{array}$ & $\begin{array}{l}b \\
\text { (o) } \\
\text { (4) }\end{array}$ & $\begin{array}{l}d \\
(\mathrm{pc}) \\
(5)\end{array}$ & $\begin{array}{l}\text { sp. } \\
\text { (6) }\end{array}$ & $\begin{array}{l}V \\
\left(\mathrm{~km} \mathrm{~s}^{-1}\right) \\
(7)\end{array}$ & $\begin{array}{l}\delta V \\
\left(\mathrm{~km} \mathrm{~s}^{-1}\right) \\
(8)\end{array}$ & $\begin{array}{l}\text { Ref. } \\
\text { (9) }\end{array}$ & $\begin{array}{l}\text { Comments } \\
\text { (10) }\end{array}$ \\
\hline & 141180 & 344.4 & +20.7 & 358 & & $-9.3,+1.7,+17.8$ & 4 & 6 & \\
\hline & \multirow[t]{7}{*}{141637} & 346.1 & +21.7 & $160 \mathrm{~h}$ & Ca II & +1.0 & 3.6 & 4 & \\
\hline & & & & & & $-3.6,-0.4,+0.3,+1.0,+2.3,+3.0$ & 0.8 & 16 & \\
\hline & & & & & $\mathrm{NaI}$ & $-6.7,+1.0$ & 3.6 & 4 & \\
\hline & & & & & & $-8.2,-5.2,-3.5,+0.1,+2.0$ & 0.5 & 15 & \\
\hline & & & & & & $-8,-5,0,+2$ & 0.8 & 16 & \\
\hline & & & & & & $-7.3,-4.3,+1.7,+2.7$ & 0.5 & 47 & \\
\hline & & & & & & $-7.9,+0.4,+2.4$ & 1.1 & 33 & \\
\hline & \multirow[t]{3}{*}{142114} & 346.9 & +21.6 & $133 \mathrm{~h}$ & $\mathrm{Ca}$ II & -8.1 & 3.6 & 4 & \\
\hline & & & & & $\mathrm{NaI}$ & $-8.1,-3.1$ & 4 & 4 & \\
\hline & & & & & & -9.0 & 6 & 5 & \\
\hline & \multirow[t]{5}{*}{143018} & 347.2 & +20.2 & $141 \mathrm{~h}$ & & $-13.3,-8.0,-7.0,-6.0,-5.3,-3.7$, & 0.5 & 15 & \\
\hline & & & & & & +1.8 & 0.5 & 15 & \\
\hline & & & & & & $-14,-8,-6,-3,+2$ & 0.8 & 16 & \\
\hline & & & & & K I & +8.9 & 8 & 34 & \\
\hline & & & & & $\mathrm{NaI}$ & $-12.6,-6.1,-4.1$ & 0.5 & 47 & \\
\hline & \multirow[t]{3}{*}{143275} & 350.1 & +22.5 & $123 \mathrm{~h}$ & $\mathrm{Ca} I I$ & $-5,-2$ & 1.0 & 48 & \\
\hline & & & & & K I & $-4.9,-1.0$ & 1.1 & 33 & \\
\hline & & & & & $\mathrm{NaI}$ & $-11.6,-2.1,+4.9$ & 0.5 & 47 & \\
\hline & \multirow[t]{9}{*}{144470} & 352.8 & +22.8 & $130 \mathrm{~h}$ & $\mathrm{Ca}$ II & $-13.4,+1.1$ & 3.6 & 4 & \\
\hline & & & & & & +1.1 & 6.0 & 5 & \\
\hline & & & & & & +3 & 1.0 & 48 & \\
\hline & & & & & Ti II & $-21,+3,+11$ & 3.7 & 14 & \\
\hline & & & & & $\mathrm{NaI}$ & $-13.4,-5.4,+1.6$ & 4 & 4 & \\
\hline & & & & & & -0.4 & 6 & 5 & \\
\hline & & & & & K I & $-1.9,-0.5,+1.0,+2.2,+4.7$ & 1 & 33 & \\
\hline & & & & & $\mathrm{NaI}$ & $-12.9,-5.9,-1.4$ & 0.5 & 47 & \\
\hline & & & & & & +2.6 & 4.0 & 47 & \\
\hline & 144844 & 350.7 & +20.4 & $131 \mathrm{~h}$ & & $-12.6,-0.2$ & 5 & 40 & \\
\hline & 145102 & 348.6 & +17.9 & $175 \mathrm{~h}$ & & $-12.0,+3.8$ & 4.0 & 6 & \\
\hline & 145482 & 348.1 & +16.8 & $143 \mathrm{~h}$ & & $-10.3,+7.5$ & 5 & 40 & \\
\hline & 146284 & 351.6 & +18.7 & $264 \mathrm{~h}$ & & $-14.2,+1.6$ & 4.0 & 6 & \\
\hline & 147084 & 352.3 & +18.1 & 170 & K I & $-1.7,+2.3,+2.9,+3.9$ & 1.1 & 33 & \\
\hline & \multirow[t]{8}{*}{147165} & 351.3 & +17.0 & $225 \mathrm{~h}$ & Ti II & $-5,+5$ & 3.7 & 14 & \\
\hline & & & & & $\mathrm{Ca}$ II & +4 & 1.0 & 48 & \\
\hline & & & & & & $-20.9,-17.9,-11.6,-6.9,-2.3$ & 0.8 & 16 & \\
\hline & & & & & & $+1.5,+3.5,+4.9,+6.1$ & 0.8 & 16 & \\
\hline & & & & & $\mathrm{NaI}$ & -3.7 & 0.5 & 47 & \\
\hline & & & & & & +4.3 & 6 & 47 & \\
\hline & & & & & K I & $+3.4,+5.1,+6.6$ & 1.1 & 33 & \\
\hline & & & & & & +2.7 & 8 & 34 & \\
\hline & 147889 & 352.9 & +17.0 & $136 \mathrm{~h}$ & & +2.6 & 8 & 34 & \\
\hline & \multirow[t]{3}{*}{$147933 / 4$} & 353.7 & +17.7 & 149 & & +1.9 & 8 & 34 & ref.54 \\
\hline & & & & & & +2.7 & 1.8 & 35 & \\
\hline & & & & & & $-0.7,+0.1,+1.5,+2.5,+3.7,+4.3$ & 1.1 & 33 & \\
\hline & \multirow[t]{2}{*}{148605} & 353.1 & +15.8 & $120 \mathrm{~h}$ & & +2.6 & 1.1 & 33 & \\
\hline & & & & & & +10.1 & 8 & 34 & \\
\hline & NSV6981 & 342.7 & +32.0 & 35 & $\mathrm{NaI}$ & $-12.2,+2.7$ & 4.6 & 17 & \\
\hline \multirow[t]{8}{*}{16} & 94473 & 272.8 & +29.2 & $278 \mathrm{~d}$ & $\mathrm{NaI}$ & $-20.4,-3.4,+1.4$ & 4 & 3 & \\
\hline & 106625 & 291.0 & +44.5 & $51 \mathrm{~h}$ & $\mathrm{Ca}$ II & -4.1 & 0.7 & 16 & \\
\hline & \multirow[t]{3}{*}{116658} & 316.1 & +50.8 & $80 \mathrm{~h}$ & $\mathrm{Ca}$ II & $-8.5,-1.5,-0.1$ & 2 & 28 & \\
\hline & & & & & & $-7.1,+0.8$ & 5 & 42 & \\
\hline & & & & & $\mathrm{NaI}$ & -7.3 & 3 & 42 & \\
\hline & 117880 & 316.7 & +43.2 & $208 \mathrm{~d}$ & $\mathrm{Ca} I I$ & $-5.7,+2.2$ & 1 & 2 & \\
\hline & 119786 & 321.6 & +45.1 & $104 \mathrm{~h}$ & Ti II & +1 & 5.5 & 1 & \\
\hline & & & & & $\mathrm{NaI}$ & -8 & 5.5 & 1 & \\
\hline 17 & 140873 & 365.5 & +39.0 & $125 \mathrm{~h}$ & $\mathrm{NaI}$ & +3.3 & 5 & 40 & \\
\hline & 141569 & 364.2 & +36.9 & $99 \mathrm{~h}$ & & +0.4 & 4 & 3 & \\
\hline & & & & & $\mathrm{Ca} I I$ & $-15.1,-0.1$ & 0.3 & 8 & \\
\hline & & & & & $\mathrm{NaI}$ & -0.1 & 0.3 & 8 & \\
\hline & 142039 & 372.9 & +41.1 & 90 & & $-2.6,+17.8,+37.3$ & 4 & 3 & \\
\hline & 142930 & 373.1 & +39.7 & $132 \mathrm{~h}$ & & $-8.1,-0.7$ & 4 & 3 & \\
\hline & 153562 & 389.1 & +29.2 & $236 \mathrm{~h}$ & & $+0.5,+6.3,+15.2$ & 4 & 3 & \\
\hline 18 & 432 & 117.5 & -3.3 & $17 \mathrm{~h}$ & Mg II & +18.3 & & $52 a$ & \\
\hline & 11433 & 138.6 & -31.4 & $20 \mathrm{~h}$ & & $+16.4,+12.2$ & & $52 \mathrm{a}$ & \\
\hline
\end{tabular}


W. G. L. Pöppel et al.: The interstellar medium surrounding the Scorpius-Centaurus association revisited

Table A.1. continued.

\begin{tabular}{|c|c|c|c|c|c|c|c|c|c|}
\hline $\begin{array}{l}\mathrm{Pa}- \\
\text { nel } \\
\text { (1) }\end{array}$ & HD & $\begin{array}{l}l \\
\text { (o) } \\
\text { (3) }\end{array}$ & $\begin{array}{l}b \\
\text { (o) } \\
\text { (4) }\end{array}$ & $\begin{array}{l}d \\
(\mathrm{pc}) \\
(5)\end{array}$ & $\begin{array}{l}\text { sp. } \\
\text { (6) }\end{array}$ & $\begin{array}{l}V \\
\left(\mathrm{~km} \mathrm{~s}^{-1}\right) \\
(7)\end{array}$ & $\begin{array}{l}\delta V \\
\left(\mathrm{~km} \mathrm{~s}^{-1}\right) \\
(8)\end{array}$ & $\begin{array}{l}\text { Ref. } \\
\text { (9) }\end{array}$ & $\begin{array}{l}\text { Comments } \\
\text { (10) }\end{array}$ \\
\hline & 8538 & 127.2 & -2.4 & $31 \mathrm{~h}$ & & +18.7 & & $52 a$ & \\
\hline & 120315 & 100.7 & +65.3 & $31 \mathrm{~h}$ & & $+15.7,+10.4$ & & $52 \mathrm{a}$ & \\
\hline & 82210 & 142.6 & +38.9 & $32 \mathrm{~h}$ & & +15.4 & & $52 \mathrm{a}$ & \\
\hline & 27808 & 174.8 & -19.1 & $41 \mathrm{~h}$ & & +12.2 & & $52 \mathrm{a}$ & \\
\hline & 26345 & 175.2 & -23.6 & $43 \mathrm{~h}$ & & +9.8 & & $52 \mathrm{a}$ & \\
\hline & 29419 & 176.0 & -15.6 & $44 \mathrm{~h}$ & & +12.3 & & $52 \mathrm{a}$ & \\
\hline & 28033 & 175.4 & -18.9 & $46 \mathrm{~h}$ & & +12.6 & & $52 \mathrm{a}$ & \\
\hline & 220657 & 98.6 & -35.4 & $53 \mathrm{~h}$ & & $+16.5,+8.3$ & & $52 \mathrm{a}$ & \\
\hline & 203280 & 101.0 & +9.2 & $15 \mathrm{~h}$ & $\mathrm{Ca}$ II & +14.3 & & $52 \mathrm{a}$ & \\
\hline & 358 & 111.7 & -32.8 & $30 \mathrm{~h}$ & & $+17.6,+13.5$ & & $52 \mathrm{a}$ & \\
\hline & 8538 & 127.2 & -2.4 & $31 \mathrm{~h}$ & & $+18.6,+12.9,+17.3$ & & $52 \mathrm{a}$ & \\
\hline & 120315 & 100.7 & +65.3 & $31 \mathrm{~h}$ & & +9.7 & & $52 \mathrm{a}$ & \\
\hline & 213558 & 101.3 & -6.6 & $31 \mathrm{~h}$ & & $+15.5,+10.3$ & & $52 \mathrm{a}$ & \\
\hline & 112413 & 118.3 & +78.8 & $34 \mathrm{~h}$ & & +7.4 & & $52 \mathrm{a}$ & \\
\hline & 222439 & 109.8 & -16.7 & $52 \mathrm{~h}$ & & $+9.1,+15.9$ & & $52 \mathrm{a}$ & \\
\hline & 32630 & 165.4 & +0.3 & $67 \mathrm{~h}$ & & $+11.8,+17.0, \ldots$ & & $52 \mathrm{a}$ & \\
\hline \multirow[t]{21}{*}{19} & 144218 & 353.2 & +23.6 & $74 ?$ & $\mathrm{NaI}$ & -12.7 & 5 & 40 & \\
\hline & 144470 & 352.8 & +22.8 & $130 \mathrm{~h}$ & & -13.4 & 3.6 & 4 & \\
\hline & & & & & Ca II & -13.4 & 3.6 & 4 & \\
\hline & 148184 & 357.9 & +20.7 & $150 \mathrm{~h}$ & $\mathrm{NaI}$ & -14.2 & 3.6 & 4 & \\
\hline & & & & & Ca II & -15.2 & 3.6 & 4 & \\
\hline & & & & & $\mathrm{NaI}$ & -12.2 & 0.5 & 47 & \\
\hline & 143018 & 347.2 & +20.2 & $141 \mathrm{~h}$ & & -13.3 & 0.5 & 15 & \\
\hline & & & & & & -14 & 0.8 & 16 & \\
\hline & 144217 & 353.2 & +23.6 & $163 \mathrm{~h}$ & & $-12.6,-11.2$ & 0.5 & 15 & \\
\hline & & & & & Ca II & -13.2 & 0.8 & 16 & \\
\hline & & & & & $\mathrm{NaI}$ & $-13,-11$ & 0.8 & 16 & \\
\hline & & & & & & -12.6 & 5 & 40 & \\
\hline & 145482 & 348.1 & +16.8 & $143 \mathrm{~h}$ & & -10.3 & 5 & 40 & \\
\hline & 145102 & 348.6 & +17.9 & $175 \mathrm{~h}$ & & -12.0 & 4.0 & 6 & \\
\hline & 144844 & 350.7 & +20.4 & $131 \mathrm{~h}$ & & -12.6 & 5 & 40 & \\
\hline & 145502 & 354.6 & +22.7 & $134 \mathrm{~h}$ & & -14.7 & 5 & 40 & \\
\hline & & & & & & -13.4 & 0.5 & 47 & \\
\hline & 147165 & 351.3 & +17.0 & $225 \mathrm{~h}$ & Ca II & -11.6 & 0.8 & 16 & \\
\hline & 143275 & 350.1 & +22.5 & $123 \mathrm{~h}$ & $\mathrm{NaI}$ & -11.6 & 0.5 & 47 & \\
\hline & 142983 & 356.4 & +28.6 & $157 \mathrm{~h}$ & & -12.6 & 3.6 & 4 & \\
\hline & & & & & Ca II & -13.1 & 3.6 & 4 & \\
\hline
\end{tabular}

Comments. 1: clear signal read off from the published plot although not mentioned by the authors.

References: 1, Albert, C. E. 1983, ApJ, 272, 509; 2, Albert, C. E., Blades, J. C., Morton, D. C., et al. 1993, ApJS, 88, 81;

3, Penprase, B. E. 1993, ApJS, 88, 433; 4, Crawford, I. A. 1991a, A\&A, 247, 183; 1991b, MNRAS, 250, 707;

5, Crawford, I. A. 1992a, MNRAS, 259, 47; 6, Crawford, I. A. 2000, MNRAS, 317, 996;

7, Welsh, B. Y., Sasseen, T., Craig, N., et al. 1997, ApJS, 112, 507; 8, Sahu, M. S., Blades, J. C., He, L., et al. 1998, ApJ, 504, 522;

10, Covino, E., Palazzi, E., Penprase, B. E., et al. 1997, A\&AS, 122, 95; 11, Penprase, B. E., Lauer, J., Aufrecht, J., et al. 1998, ApJ, 492, 617;

12, Penprase, B. E., \& Blades, J. C. 2000, ApJ, 535, 293; 14, Stokes, G. M. 1978, ApJS, 36, 115;

15, Welty, D. E., Hobbs, L. M., \& Kulkarni, V. P. 1994, ApJ, 436, 152; 16, Welty, D. E., Morton, D. C., \& Hobbs, L. M. 1996, ApJS, 106, 533;

17, Welsh, B. Y., Craig, N., Vedder, P. W., et al. 1994, ApJ, 437, 638; 28, Vallerga, J. V., Vedder, P. W., Craig, N., et al. 1993, ApJ, 411, 729;

33, Welty, D. E., \& Hobbs, L. M. 2001, ApJS, 133, 345; 34, Chaffee, F. H., \& White, R. E. 1982, ApJS, 50, 169;

35, Lauroesch, J. T., Meyer, D. M., Watson, J. K., et al. 1998, ApJ, 507, L89;

36, Sembach, K. R., Danks, A. C., \& Lambert, D. L. 1996, ApJ, 460, L61;

38, Cha, A. N., Sahu, M. S., Warren Moos, H., et al. 2000, ApJS, 129, 281;

40, Génova, R., Beckman, J. E., Bowyer, S., et al. 1997, ApJ, 484, 761; 42, Centurión, M., \& Vladilo, G. 1991, ApJ, 372, 494;

43, Crawford, I. A., Craig, N., \& Welsh, B. Y. 1997, A\&A, 317, 889; 46, Lyons, M. A., Bates, B., \& Kemp, S. N. 1994, A\&A, 286, 535;

47, Hobbs, L. M. 1969, ApJ, 157, 175; 48, Marshall, L. A., \& Hobbs, L. M. 1972, ApJ, 173, 43;

52a, Redfield, S., \& Linsky, J. L. 2002, ApJS, 139, 439; 52c, Lallement, R., Vidal-Madjar, A., \& Ferlet, R. 1986, A\&A, 168, 225;

54, Diplas, A., \& Savage, B. D. 1994, ApJS, 93, 211; 55, Corradi, W. J. B., Franco, G. A. P., \& Knude, J. 2004, MNRAS, $347,1065$. 

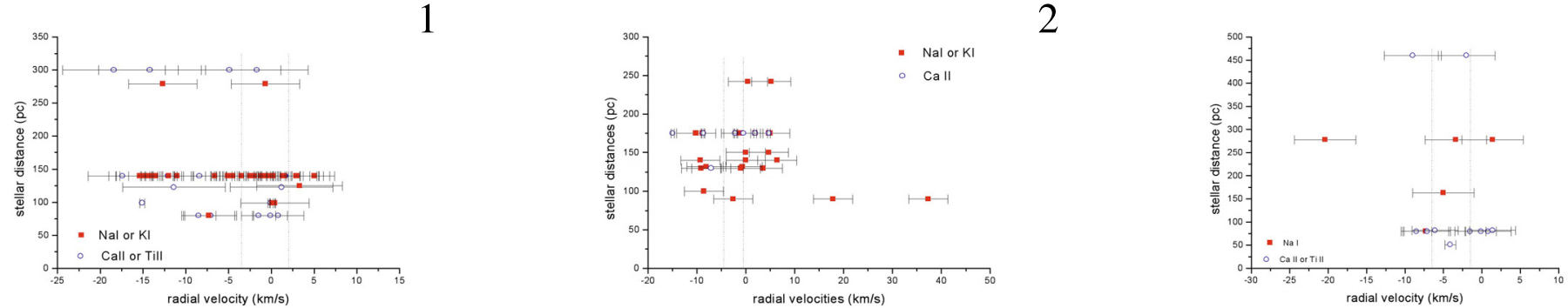

4
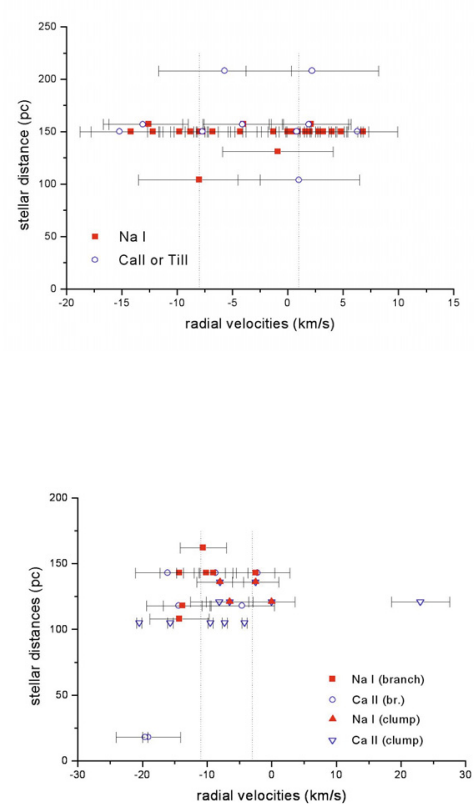

10

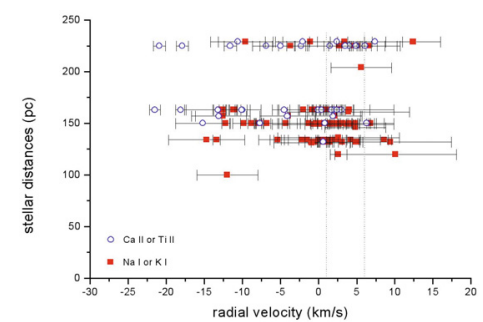

13

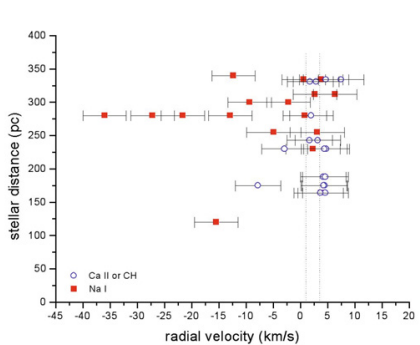

11

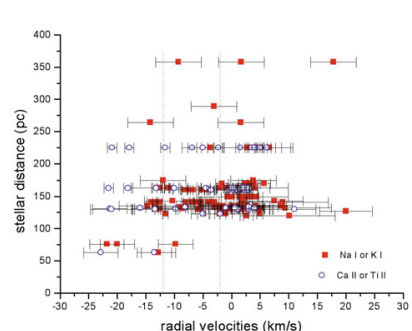

8

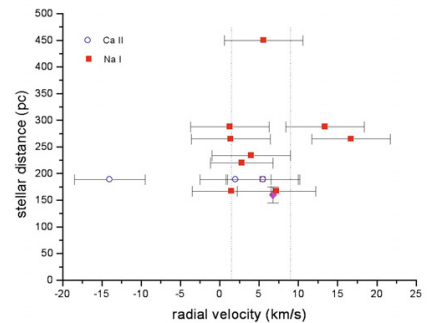

5

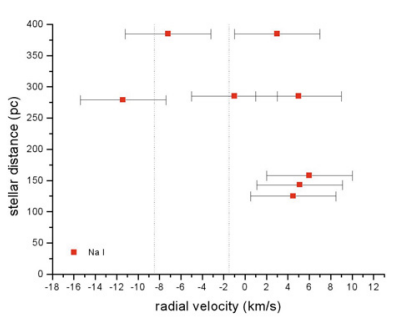

6
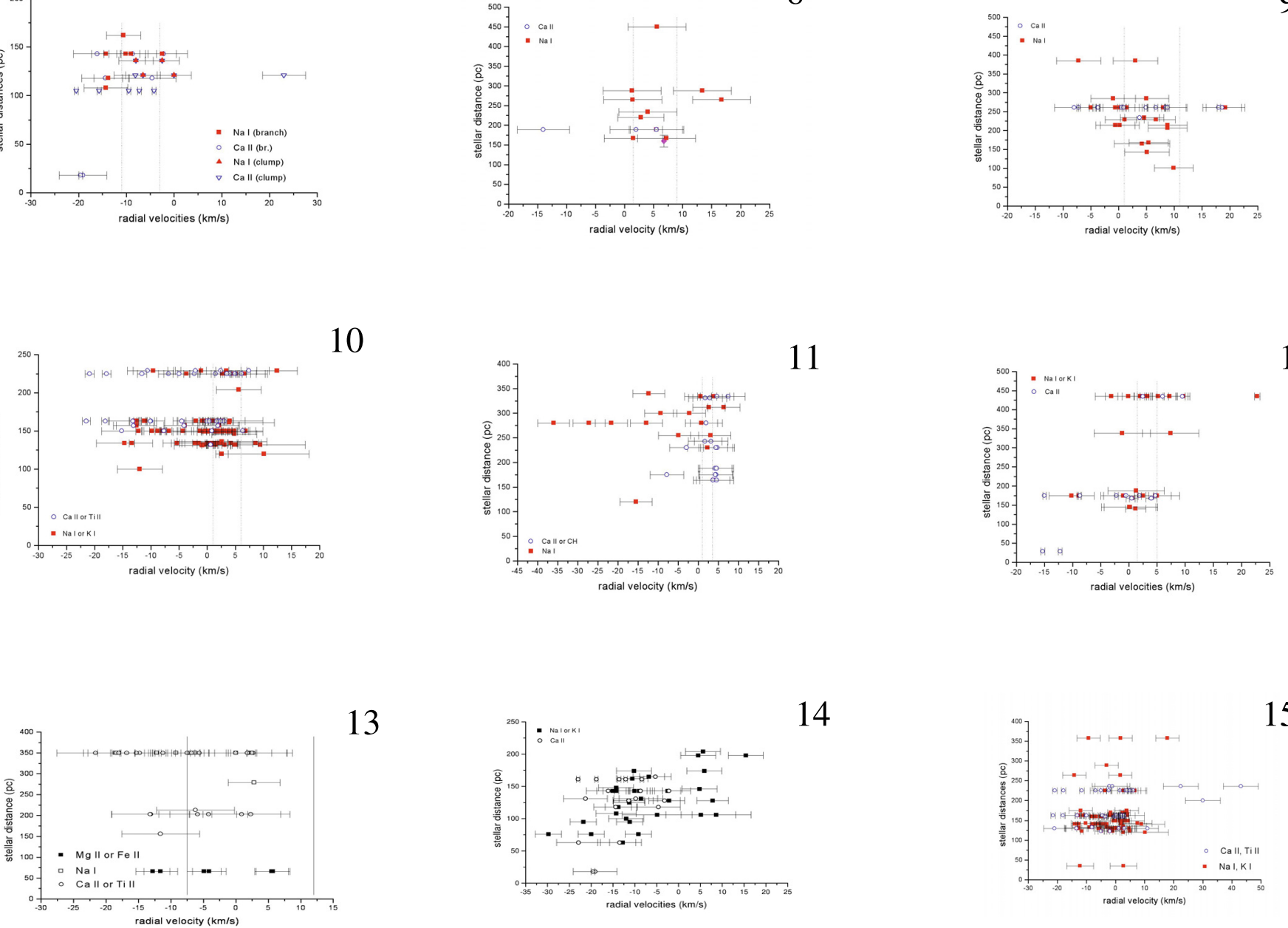

Fig. A.1. Plots of the radial velocities $V\left(\mathrm{~km} \mathrm{~s}^{-1}\right)$ of optical IS components in abscissas, as a function of the stellar distances $d$ (pc) in ordinates. The data were derived from the results of observations published in the literature. The dotted lines in panels $1-13$ correspond to the velocity ranges plotted in Fig. 6. The velocity resolutions are used as error bars. For each plot the data and the corresponding references are listed by separate in Table A.1. See the text for more details. 